EDITORIAL BOARD

ANTONIO CARCATERRA

ERIC A. CARLEN

FRANCESCO DELL'ISOLA

RAFFAELE ESPOSITO

ALBERT FANNJIANG

Gilles A. FranCFORT

Pierangelo MARCATI

JEAN-JACQUES MARIGO

PETER A. MARKOWICH

MARTIN OSTOJA-STARZEWSKI

PIERRE SEPPECHER

DAVID J. STEIGMANN

PAUl STEINMANN

PierRe M. SuQueT

MANAGING EDITORS

MICOL AMAR

CORRADO LATTANZIO

ANGELA MADEO

MARTIN OSTOJA-STARZEWSKI

\section{ADVISORY BOARD}

ADNAN AKAY

Holm AltenBaCH

MICOL AMAR

HARM ASKES

TEODOR ATANACKOVIĆ

VICTOR BERDICHEVSKY

GUY BOUCHITTÉ

ANDREA BRAIDES

ROBERTO CAMASSA

MAURO CARFORE

ERIC DARVE

FELIX DARVE

ANNA DE MASI

GianPiEtro DEL Piero

EMMANUELE Di BENEDETTO

BERNOLD FIEDLER

IRENE M. GAMBA

DAVID Y. GAO

SERGEY GAVRILYUK

TIMOTHY J. HEALEY

DOMINIQUE JEULIN

ROGER E. KHAYAT

CORRADO LATTANZIO

ROBERT P. LIPTON

ANGELO LUONGO

ANGELA MADEO

JUAN J. MANFREDI

CARLO MARCHIORO

GÉRARD A. MAUGIN

ROBERTO NATALINI PATRIZIO NEFF

ANDREY PIATNITSKI

ERRICO PRESUTTI

MARIO PULVIRENTI

LUCIO RUSSO

Miguel A. F. SANJUAN

PATRICK SElvadurai

ALEXANDER P. SEYRANIAN

MIROSLAV ŠILHAVÝ

GUIDO SWEERS

ANTOINETTE TORDESILLAS

LEV TRUSKINOVSKY

JUAN J. L. VELÁZQUEZ VINCENZO VESPRI ANGELO VULPIANI msp.org/memocs

Università di Roma “La Sapienza”, Italia

Rutgers University, USA

(CO-CHAIR) Università di Roma "La Sapienza", Italia

(TREASURER) Università dell'Aquila, Italia

University of California at Davis, USA

(CO-CHAIR) Université Paris-Nord, France

Università dell'Aquila, Italy

École Polytechnique, France

DAMTP Cambridge, UK, and University of Vienna, Austria

(CHAIR MANAGING EDITOR) Univ. of Illinois at Urbana-Champaign, USA

Université du Sud Toulon-Var, France

University of California at Berkeley, USA

Universität Erlangen-Nürnberg, Germany

LMA CNRS Marseille, France

Università di Roma "La Sapienza", Italia

Università dell'Aquila, Italy

Université de Lyon-INSA (Institut National des Sciences Appliquées), France

(CHAIR MANAGING EDITOR) Univ. of Illinois at Urbana-Champaign, USA

Carnegie Mellon University, USA, and Bilkent University, Turkey

Otto-von-Guericke-Universität Magdeburg, Germany

Università di Roma "La Sapienza", Italia

University of Sheffield, UK

University of Novi Sad, Serbia

Wayne State University, USA

Université du Sud Toulon-Var, France

Università di Roma Tor Vergata, Italia

University of North Carolina at Chapel Hill, USA

Università di Pavia, Italia

Stanford University, USA

Institut Polytechnique de Grenoble, France

Università dell'Aquila, Italia

Università di Ferrara and International Research Center MEMOCS, Italia

Vanderbilt University, USA

Freie Universität Berlin, Germany

University of Texas at Austin, USA

Federation University and Australian National University, Australia

Université Aix-Marseille, France

Cornell University, USA

École des Mines, France

University of Western Ontario, Canada

Università dell' Aquila, Italy

Louisiana State University, USA

Università dell'Aquila, Italia

Université de Lyon-INSA (Institut National des Sciences Appliquées), France University of Pittsburgh, USA

Università di Roma "La Sapienza”, Italia

Université Paris VI, France

Istituto per le Applicazioni del Calcolo "M. Picone", Italy

Universität Duisburg-Essen, Germany

Narvik University College, Norway, Russia

Università di Roma Tor Vergata, Italy

Università di Roma "La Sapienza”, Italia

Università di Roma “Tor Vergata”, Italia

Universidad Rey Juan Carlos, Madrid, Spain

McGill University, Canada

Moscow State Lomonosov University, Russia

Academy of Sciences of the Czech Republic

Universität zu Köln, Germany

University of Melbourne, Australia

École Polytechnique, France

Bonn University, Germany

Università di Firenze, Italia

Università di Roma La Sapienza, Italia

MEMOCS (ISSN 2325-3444 electronic, 2326-7186 printed) is a journal of the International Research Center for the Mathematics and Mechanics of Complex Systems at the Università dell'Aquila, Italy.

Cover image: "Tangle” by $\odot$ John Horigan; produced using the Context Free program (contextfreeart.org).

PUBLISHED BY

7 mathematical sciences publishers

nonprofit scientific publishing

http://msp.org/

(C) 2017 Mathematical Sciences Publishers 


\title{
ON THE WELL-POSEDNESS OF THE GREEN-LINDSAY MODEL
}

\author{
Gia Avalishvili, Mariam Avalishvili and Wolfgang H. Müller
}

\begin{abstract}
The present paper is devoted to an investigation of a nonclassical model for inhomogeneous anisotropic thermoelastic bodies with two constant relaxation times originally presented by Green and Lindsay. A variational formulation of the initial-boundary value problem corresponding to the linear dynamical three-dimensional Green-Lindsay model is applied. The corresponding spaces of vector-valued distributions with respect to the time variable with values in Sobolev spaces are defined and the existence and uniqueness of the solution in these spaces as well as continuous dependence of the solution on the given data is shown.
\end{abstract}

\section{Introduction}

The physically unrealistic feature of classical thermoelasticity, which is based on Fourier's law of heat conduction, and according to which heat spreads infinitely fast, was refuted by several experimental studies, where it was shown that heat propagates as a thermal wave at finite speed at low temperatures [Ackerman and Overton 1969; Caviglia et al. 1992; Coleman and Newman 1988; McNelly et al. 1970; Narayanamurti and Dynes 1972]. In various modern engineering constructions, such as high-speed aircraft, nuclear reactors, and recently developed ultrafast pulsed lasers, temperatures and temperature gradients are extremely high and the operation time periods are of the order of picoseconds. This results in thermal shocks and cannot be successfully described by the classical theory of thermoelasticity [Abdallah 2009; Dreyer and Struchtrup 1993; Wang and Xu 2002; Zhu et al. 1999]. Furthermore, mathematical models of propagation of heat as a thermal wave are used in order to describe various processes involving heat transfer, such as during chemotaxis [Dolak and Hillen 2003], in food technology [Saidane et al. 2005], in biological tissues [Afrin et al. 2011], in one of Saturn's moons [Bargmann et al. 2008], and in nanofluids [Vadasz et al. 2005].

\section{Communicated by Francesco dell'Isola.}

MSC2010: 74F05, 74H20, 74H25.

Keywords: Nonclassical thermoelasticity, initial-boundary value problem, existence and uniqueness of solution. 
One of the theories devoted to eliminating the shortcomings of classical thermoelasticity was presented by Müller [1971] without making any assumptions regarding the form of the heat conduction law, and admitted a finite speed of propagation of thermal waves. By applying a similar approach and by using the entropy production inequality of Green and Laws [1972], a simpler and more explicit version of nonclassical thermoelasticity was presented by Green and Lindsay [1972], which also allows for finite speeds of thermal waves. In this theory, the classical forms of the entropy flux and entropy source are preserved and, as in Müller's theory, the temperature rate is included among the constitutive variables. Note that in the Green-Lindsay theory for materials with a center of symmetry at each point the classical Fourier law is satisfied.

In the nonclassical theory of thermoelasticity developed by Green and Lindsay, the constitutive relations for the stress tensor and the entropy are generalized by introducing two different relaxation times. A uniqueness theorem for the GreenLindsay model in the case of a thermoelastic body consisting of a homogeneous material with an initial center of symmetry requiring only the usual symmetry properties of the elastic stiffness tensor was obtained by Green [1972]. The continuous dependence of the classical solution on given data and the existence of a generalized solution for initial-boundary value problems corresponding to the Green-Lindsay model (under the assumption that there is a classical solution of the problem) were proved for homogeneous isotropic thermoelastic bodies by Bem [1983]. By applying the method of potentials and the theory of integral equations, the problems of stable and pseudo-oscillations for the Green-Lindsay nonclassical model were studied by Burchuladze and Gegelia [1985]. For the Green-Lindsay nonclassical model, the problem of propagation of a thermoelastic wave was studied, and domain of influence results were obtained for a thermoelastic body consisting of homogeneous material with an initial center of symmetry by Carbonaro and Ignaczak [1987] in classical spaces of twice continuously differentiable functions.

Existence, uniqueness, and continuous dependence of the solution of the initialboundary value problem corresponding to the Green-Lindsay model with Dirichlet boundary conditions for a temperature vanishing on the entire boundary in suitable function spaces were proved in [Karakostas and Massalas 1991]. For the GreenLindsay nonclassical model, problems of wave propagation, methods of solution of the corresponding initial and initial-boundary value problems, and applications of the obtained results and related topics have been considered by many researchers (see [Chandrasekharaiah 1986; 1998; Hetnarski and Ignaczak 2000; Joseph and Preziosi 1989; Ignaczak and Ostoja-Starzewski 2010; Straughan 2011]).

It should be pointed out that three-dimensional initial-boundary value problems with general mixed boundary conditions for displacement and temperature corresponding to the linear Green-Lindsay dynamical model for an inhomogeneous 
anisotropic thermoelastic body have not been investigated yet. The well-posedness results are mainly obtained for the case of purely Dirichlet or Neumann types of boundary conditions. The initial-boundary value problem with mixed boundary conditions corresponding to the Green-Lindsay linear model for a homogeneous isotropic thermoelastic plate was investigated in first-order Sobolev spaces in the paper [Avalishvili et al. 2010] by applying a variational approach. In the present paper, we investigate the well-posedness of the linear three-dimensional initialboundary value problem corresponding to the Green-Lindsay model with general mixed boundary conditions, provided that on certain parts of the boundary of the space domain surface force and heat flux along the outward normal vector are prescribed and on the remaining parts displacement and temperature vanish. We obtain new existence, uniqueness, and continuous dependence results in the corresponding Sobolev spaces.

In Section 2, we consider a differential formulation of the initial-boundary value problem corresponding to the Green-Lindsay linear dynamical three-dimensional model for an inhomogeneous anisotropic thermoelastic body and obtain integral equations that are equivalent to the original problem in spaces of sufficiently smooth functions. On the basis of these integral equations, we present a variational formulation of the three-dimensional problem in corresponding spaces of vector-valued distributions with respect to the time variable with values in Sobolev spaces. Furthermore, we formulate results regarding the existence and uniqueness of the solution of the three-dimensional initial-boundary value problem, and regard the continuous dependence of the solution on given data in suitable function spaces.

\section{Well-posedness of the Green-Lindsay model}

In this paper we denote for each real $s \geq 0$ by $H^{s}(\Omega)$ and $H^{s}(\check{\Gamma})$ the Sobolev spaces of real-valued functions based on $H^{0}(\Omega)=L^{2}(\Omega)$ and $H^{0}(\check{\Gamma})=L^{2}(\check{\Gamma})$, respectively, where $\Omega \subset \mathbb{R}^{n}, n \in \mathbb{N}$, is a bounded Lipschitz domain and $\check{\Gamma}$ is an element of a Lipschitz dissection of the boundary $\Gamma=\partial \Omega$ [McLean 2000]. We refer to the corresponding spaces of vector-valued functions by

$$
\boldsymbol{H}^{s}(\Omega)=\left[H^{s}(\Omega)\right]^{3}, \quad \boldsymbol{H}^{s}(\check{\Gamma})=\left[H^{s}(\check{\Gamma})\right]^{3}(s \geq 0), \quad \boldsymbol{L}^{s_{1}}(\check{\Gamma})=\left[L^{s_{1}}(\check{\Gamma})\right]^{3}\left(s_{1} \geq 1\right)
$$

and by $\operatorname{tr}: \boldsymbol{H}^{1}(\Omega) \rightarrow \boldsymbol{H}^{1 / 2}(\Gamma)$ and $\operatorname{tr}: H^{1}(\Omega) \rightarrow H^{1 / 2}(\Gamma)$ the trace operators. $C^{0,1}(\bar{\Omega})$ characterizes the space of Lipschitz continuous function on $\bar{\Omega}$. For any measurable set $D,(\cdot, \cdot)_{L^{2}(D)}$ and $(\cdot, \cdot)_{L^{2}(D)}$ are the classical scalar products in $\boldsymbol{L}^{2}(D)$ and $L^{2}(D)$, respectively. For a Banach space $X, C([0, T] ; X)$ is the space of continuous vector functions on $[0, T]$ with values in $X . L^{m}(0, T ; X), 1 \leq$ $m \leq \infty$, is the space of such measurable vector functions $g:(0, T) \rightarrow X$ that $\|g\|_{X} \in L^{m}(0, T)$, and the generalized derivative of $g$ we denote by $g^{\prime}=\mathrm{d} g / \mathrm{d} t \in$ 
$\mathfrak{D}^{\prime}(0, T ; X)$ [Dautray and Lions 1992]. If $g \in L^{1}(0, T ; X)$ and $X$ is a space of functions of variable $x \in \Omega$, then we identify $g$ with a function $g(x, t)$ and $g(t)$ denotes the function $g(t): x \rightarrow g(x, t)$, for almost all $t \in(0, T)$. The distributional derivative $\mathrm{d} g / \mathrm{d} t$ we identify with the derivative $\partial g / \partial t$ of $g$ in $\mathfrak{D}^{\prime}(\Omega \times(0, T))$.

Let us consider a thermoelastic body with initial configuration $\bar{\Omega}$ consisting of general inhomogeneous anisotropic thermoelastic material, which is described by the Green-Lindsay linear dynamical three-dimensional model [Green and Lindsay 1972], and whose thermal and elastic properties are characterized by the following consistently spatially dependent thermoelastic parameters:

(a) an elasticity tensor $\mu_{i j p q}(x), x \in \Omega(i, j, p, q=1,2,3)$, which satisfies the symmetry and positive definiteness conditions

$$
\begin{gathered}
\mu_{i j p q}(x)=\mu_{p q i j}(x)=\mu_{j i p q}(x) \quad \forall x \in \Omega, \\
\sum_{i, j, p, q=1}^{3} \mu_{i j p q}(x) \varepsilon_{p q} \varepsilon_{i j} \geq c_{\mu} \sum_{i, j=1}^{3}\left(\varepsilon_{i j}\right)^{2} \quad \forall \varepsilon_{i j} \in \mathbb{R}, x \in \Omega,
\end{gathered}
$$

where $c_{\mu}$ is a constant $>0$ and $\varepsilon_{i j}=\varepsilon_{j i}$;

(b) a mass density $\rho(x), x \in \Omega$;

(c) a thermal conductivity tensor $\lambda_{p q}(x), x \in \Omega(p, q=1,2,3)$, which satisfies the following symmetry and positive definite conditions:

$$
\begin{gathered}
\lambda_{p q}(x)=\lambda_{q p}(x) \quad \forall x \in \Omega, \\
\sum_{p, q=1}^{3} \lambda_{p q}(x) \varepsilon_{p} \varepsilon_{q} \geq c_{\lambda} \sum_{p=1}^{3}\left(\varepsilon_{p}\right)^{2} \quad \forall \varepsilon_{p} \in \mathbb{R}, x \in \Omega,
\end{gathered}
$$

where $c_{\lambda}$ is a constant $>0$;

(d) a thermal capacity $\varkappa(x), x \in \Omega$;

(e) a stress-temperature tensor $\eta_{p q}(x)$, and thermal coefficients $\beta_{p}(x), x \in \Omega$ $(p, q=1,2,3)$, such that

$$
\eta_{p q}(x)=\eta_{q p}(x) \quad \forall x \in \Omega
$$

(f) relaxation times $\tau_{0}=$ const $>0$ and $\tau_{1}=$ const $>0$;

(g) temperature of thermoelastic body in natural state $\Theta_{0}=$ const $>0$, which is considered as a reference temperature.

It should be noted that the constraint of constant relaxation times will be removed in a forthcoming paper [Avalishvili et al. 2017].

We consider mixed boundary conditions on the boundary $\Gamma=\partial \Omega$ of the thermoelastic body, such that on certain parts of the boundary the displacement or the 
temperature vanishes, and on the remaining parts the stress vector or the heat flux along the outward normal of the boundary are given. We assume that the body is clamped along a part $\Gamma_{0} \subset \Gamma$ and that the temperature $\theta$ vanishes along a part $\Gamma_{0}^{\theta} \subset \Gamma$. The body is subjected to:

(i) an applied body force with density $\boldsymbol{f}=\left(f_{i}\right): \Omega \times(0, T) \rightarrow \mathbb{R}^{3}$;

(ii) an applied surface force with density $g=\left(g_{i}\right): \Gamma_{1} \times(0, T) \rightarrow \mathbb{R}^{3}$ is given along the part $\Gamma_{1}=\Gamma \backslash \bar{\Gamma}_{0}$ of the boundary of $\Omega$, where $\partial \Omega=\Gamma_{0} \cup \Gamma_{01} \cup \Gamma_{1}$, $\Gamma_{0} \cap \Gamma_{1}=\varnothing$, is a Lipschitz dissection of $\partial \Omega$ [McLean 2000];

(iii) a heat source with density $f^{\theta}: \Omega \times(0, T) \rightarrow \mathbb{R}$;

(iv) a heat flux with density $g^{\theta}: \Gamma_{1}^{\theta} \times(0, T) \rightarrow \mathbb{R}$ along the outward normal vector of $\Gamma$, which is given on $\Gamma_{1}^{\theta}=\Gamma \backslash \Gamma_{1}^{\theta}$, where $\partial \Omega=\Gamma_{0}^{\theta} \cup \Gamma_{01}^{\theta} \cup \Gamma_{1}^{\theta}, \Gamma_{0}^{\theta} \cap \Gamma_{1}^{\theta}=\varnothing$, is a Lipschitz dissection of $\partial \Omega$.

The dynamical linear three-dimensional model for the stress-strain state of a thermoelastic body $\Omega$ obtained by Green and Lindsay [1972] is given by the following initial-boundary value problem in differential form:

$$
\begin{gathered}
\rho \frac{\partial^{2} u_{i}}{\partial t^{2}}=\sum_{j=1}^{3} \frac{\partial}{\partial x_{j}}\left(\sum_{p, q=1}^{3} \mu_{i j p q} e_{p q}(\boldsymbol{u})+\eta_{i j} \theta+\eta_{i j} \tau_{1} \frac{\partial \theta}{\partial t}\right)+f_{i} \text { in } \Omega \times(0, T), \\
\varkappa\left(\frac{\partial \theta}{\partial t}+\tau_{0} \frac{\partial^{2} \theta}{\partial t^{2}}\right)-\sum_{p=1}^{3} \beta_{p} \frac{\partial^{2} \theta}{\partial t \partial x_{p}}=\sum_{p, q=1}^{3} \frac{\partial}{\partial x_{p}}\left(\lambda_{p q} \frac{\partial \theta}{\partial x_{q}}\right)+\sum_{p=1}^{3} \frac{\partial}{\partial x_{p}}\left(\beta_{p} \frac{\partial \theta}{\partial t}\right) \\
+\Theta_{0} \sum_{p, q=1}^{3} \eta_{p q} e_{p q} \frac{\partial \boldsymbol{u}}{\partial t}+f^{\theta} \text { in } \Omega \times(0, T), \\
\boldsymbol{u}(x, 0)=\boldsymbol{u}_{0}(x), \quad \frac{\partial \boldsymbol{u}}{\partial t}(x, 0)=\boldsymbol{u}_{1}(x), \\
\theta(x, 0)=\theta_{0}(x), \quad \frac{\partial \theta}{\partial t}(x, 0)=\theta_{1}(x) \text { in } \Omega, \\
\sum_{j=1}^{3}\left(\sum_{p, q=1}^{3} \mu_{i j p q} e_{p q}(\boldsymbol{u})+\eta_{i j} \theta+\eta_{i j} \tau_{1} \frac{\partial \theta}{\partial t}\right) \nu_{j}=g_{i} \text { on } \Gamma_{1} \times(0, T), \\
\theta=0 \text { on } \Gamma_{0}^{\theta} \times(0, T), \\
-\sum_{p=1}^{3}\left(\sum_{q=1}^{3} \lambda_{p q} \frac{\partial \theta}{\partial x_{q}}+\beta_{p} \frac{\partial \theta}{\partial t}\right) v_{p}=g^{\theta} \text { on } \Gamma_{1}^{\theta} \times(0, T),
\end{gathered}
$$

where $e_{i j}(\boldsymbol{v})=\frac{1}{2}\left(\partial_{i} v_{j}+\partial_{j} v_{i}\right)(i, j=1,2,3), \boldsymbol{v}=\left(v_{i}\right)$ is the outward unit normal to $\Gamma, \boldsymbol{u}=\left(u_{i}\right): \Omega \times(0, T) \rightarrow \mathbb{R}^{3}$ is the displacement vector-function of the thermoelastic body, $\theta: \Omega \times(0, T) \rightarrow \mathbb{R}$ is the temperature distribution, $\boldsymbol{u}_{0}=\left(u_{0 i}\right)_{i=1}^{3}$ 
and $\boldsymbol{u}_{1}=\left(u_{1 i}\right)_{i=1}^{3}$ are the initial displacement and velocity vector-functions, and $\theta_{0}$ is the initial distribution of temperature.

Remark. If the thermoelastic body consists of a material that initially has a center of symmetry at each point, then parameters $\beta_{p}(p=1,2,3)$ vanish.

By multiplying (6) by arbitrary continuously differentiable functions $v_{i}: \bar{\Omega} \rightarrow \mathbb{R}$ $(i=1,2,3)$, which vanish on $\Gamma_{0}$, and (7) by a continuously differentiable function $\varphi: \bar{\Omega} \rightarrow \mathbb{R}$, such that $\varphi=0$ on $\Gamma_{0}^{\theta}$, by using Green's formula, the symmetry properties of the tensors $\mu_{i j p q}, \eta_{i j}$ and $e_{p q}(v)$, and the boundary conditions (9) and (10), we obtain the following integral equations:

$$
\begin{aligned}
& \sum_{i=1}^{3} \int_{\Omega} \rho \frac{\partial^{2} u_{i}}{\partial t^{2}} v_{i} \mathrm{~d} x+\sum_{i, j=1}^{3} \int_{\Omega} \sum_{p, q=1}^{3} \mu_{i j p q} e_{p q}(\boldsymbol{u}) e_{i j}(\boldsymbol{v}) \mathrm{d} x \\
& +\sum_{i, j=1}^{3} \int_{\Omega}\left(\eta_{i j} \theta+\eta_{i j} \tau_{1} \frac{\partial \theta}{\partial t}\right) e_{i j}(\boldsymbol{v}) \mathrm{d} x=\sum_{i=1}^{3} \int_{\Omega} f_{i} v_{i} \mathrm{~d} x+\sum_{i=1}^{3} \int_{\Gamma_{1}} g_{i} v_{i} \mathrm{~d} \Gamma, \\
& \int_{\Omega} \varkappa\left(\frac{\partial \theta}{\partial t}+\tau_{0} \frac{\partial^{2} \theta}{\partial t^{2}}\right) \varphi \mathrm{d} x-\int_{\Omega} \sum_{p=1}^{3} \beta_{p} \frac{\partial^{2} \theta}{\partial t \partial x_{p}} \varphi \mathrm{d} x+\sum_{p, q=1}^{3} \int_{\Omega} \lambda_{p q} \frac{\partial \theta}{\partial x_{q}} \frac{\partial \varphi}{\partial x_{p}} \mathrm{~d} x \\
& \quad+\sum_{p=1}^{3} \int_{\Omega} \beta_{p} \frac{\partial \theta}{\partial t} \frac{\partial \varphi}{\partial x_{p}} \mathrm{~d} x-\Theta_{0} \int_{\Omega} \sum_{p, q=1}^{3} \eta_{p q} e_{p q}\left(\frac{\partial \boldsymbol{u}}{\partial t}\right) \varphi \mathrm{d} x \\
& =\int_{\Omega} f^{\theta} \varphi \mathrm{d} x-\int_{\Gamma_{1}^{\theta}} g^{\theta} \varphi \mathrm{d} \Gamma .
\end{aligned}
$$

Therefore, if $\boldsymbol{u}=\left(u_{i}\right)_{i=1}^{3}$ and $\theta$ are solutions to (6) and (7) and satisfy the boundary conditions (9) and (10), then $\boldsymbol{u}=\left(u_{i}\right)_{i=1}^{3}$ and $\theta$ are solutions to (11) and (12). Conversely, if $\boldsymbol{u}=\left(u_{i}\right)_{i=1}^{3}$ and $\theta$ are twice continuously differentiable solutions of the integral equations (11) and (12), then we use Green's formula to obtain

$$
\begin{aligned}
\sum_{i=1}^{3} \int_{\Omega}\left(\rho \frac{\partial^{2} u_{i}}{\partial t^{2}}-\sum_{j=1}^{3} \frac{\partial}{\partial x_{j}}\left(\sum_{p, q=1}^{3} \mu_{i j p q} e_{p q}(\boldsymbol{u})+\eta_{i j} \theta+\eta_{i j} \tau_{1} \frac{\partial \theta}{\partial t}\right)\right) v_{i} \mathrm{~d} x \\
\quad+\sum_{i, j=1}^{3} \int_{\Gamma_{1}}\left(\sum_{p, q=1}^{3} \mu_{i j p q} e_{p q}(\boldsymbol{u})+\eta_{i j} \theta+\eta_{i j} \tau_{1} \frac{\partial \theta}{\partial t}\right) v_{i} v_{j} \mathrm{~d} \Gamma \\
=\sum_{i=1}^{3} \int_{\Omega} f_{i} v_{i} \mathrm{~d} x+\sum_{i=1}^{3} \int_{\Gamma_{1}} g_{i} v_{i} \mathrm{~d} \Gamma,
\end{aligned}
$$




$$
\begin{gathered}
\int_{\Omega}\left(\varkappa\left(\frac{\partial \theta}{\partial t}+\tau_{0} \frac{\partial^{2} \theta}{\partial t^{2}}\right)-\sum_{p=1}^{3} \beta_{p} \frac{\partial^{2} \theta}{\partial t \partial x_{p}}-\sum_{p, q=1}^{3} \frac{\partial}{\partial x_{p}}\left(\lambda_{p q} \frac{\partial \theta}{\partial x_{q}}\right)\right. \\
\left.-\sum_{p=1}^{3} \frac{\partial}{\partial x_{p}}\left(\beta_{p} \frac{\partial \theta}{\partial t}\right)-\Theta_{0} \sum_{p, q=1}^{3} \eta_{p q} e_{p q}\left(\frac{\partial \boldsymbol{u}}{\partial t}\right)\right) \varphi \mathrm{d} x \\
+\sum_{p=1}^{3} \int_{\Gamma_{1}^{\theta}}\left(\sum_{q=1}^{3} \lambda_{p q} \frac{\partial \theta}{\partial x_{q}}+\beta_{p} \frac{\partial \theta}{\partial t}\right) \varphi v_{p} \mathrm{~d} \Gamma \\
=\int_{\Omega} f^{\theta} \varphi \mathrm{d} x-\int_{\Gamma_{1}^{\theta}} g^{\theta} \varphi \mathrm{d} \Gamma,
\end{gathered}
$$

for all continuously differentiable functions $\boldsymbol{v}=\left(v_{i}\right)_{i=1}^{3}$ and $\varphi$ vanishing on $\Gamma_{0}$ and $\Gamma_{0}^{\theta}$, respectively. By letting $\varphi \in C_{0}^{1}(\Omega)=\left\{\psi \in C^{1}(\Omega) \mid \psi=0\right.$ on $\left.\Gamma\right\}$ and $\boldsymbol{v}=\left(v_{i}\right)_{i=1}^{3} \in\left(C_{0}^{1}(\Omega)\right)^{3}$, and by taking into account the density of $C_{0}^{1}(\Omega)$ in $L^{2}(\Omega)$ from (13) and (14) we obtain that $\boldsymbol{u}$ and $\theta$ satisfy (6) and (7). Now, if we assume that $\boldsymbol{v}=\left(v_{i}\right)_{i=1}^{3} \in\left(C_{0}^{1}\left(\Gamma_{1}\right)\right)^{3}=\left\{\boldsymbol{v}=\left(v_{i}\right)_{i=1}^{3} \in\left(C^{1}\left(\Gamma_{1}\right)\right)^{3} \mid \boldsymbol{v}=\mathbb{O}\right.$ on $\left.\Gamma_{01}\right\}$ and $\varphi \in C_{0}^{1}\left(\Gamma_{1}^{\theta}\right)=$ $\left\{\varphi \in C^{1}\left(\Gamma_{1}^{\theta}\right) \mid \varphi=0\right.$ on $\left.\Gamma_{01}^{\theta}\right\}$ are arbitrary continuous functions, then by applying (6) and (7), and the density of $C_{0}\left(\Gamma_{1}\right)$ and $C_{0}\left(\Gamma_{1}^{\theta}\right)$ being in $L^{2}\left(\Gamma_{1}\right)$ and $L^{2}\left(\Gamma_{1}^{\theta}\right)$, respectively, we infer that $\boldsymbol{u}$ and $\theta$ satisfy the boundary conditions (9) and (10).

Hence the initial-boundary value problem (6)-(10) corresponding to the GreenLindsay dynamical three-dimensional model is equivalent to the integral equations (11) and (12), together with the initial conditions (8) in the spaces of twice continuously differentiable functions. On the basis of these equations we present the so-called weak or variational formulation of the initial-boundary value problem (6)-(10), and investigate the existence and uniqueness of a weak solution in suitable spaces of vector-valued distributions with values in the corresponding Sobolev spaces.

Let us introduce the following function spaces, which are used in the variational formulation of the initial-boundary value problem (6)-(10):

$$
\begin{aligned}
\boldsymbol{V}(\Omega) & =\left\{\boldsymbol{v}=\left(v_{i}\right)_{i=1}^{3} \in \boldsymbol{H}^{1}(\Omega) ; \quad \operatorname{tr}(\boldsymbol{v})=\mathbb{0} \text { on } \Gamma_{0}\right\}, \\
V^{\theta}(\Omega) & =\left\{\varphi \in H^{1}(\Omega) ; \quad \operatorname{tr}(\varphi)=0 \text { on } \Gamma_{0}^{\theta}\right\} .
\end{aligned}
$$

Note that $\boldsymbol{V}(\Omega)$ and $V^{\theta}(\Omega)$ are Hilbert spaces equipped with the norms $\|\cdot\|_{\boldsymbol{H}^{1}(\Omega)}$ and $\|\cdot\|_{H^{1}(\Omega)}$, respectively.

We identify the unknown vector function $\boldsymbol{u}$ and the function $\theta$ with vectorfunctions defined on $[0, T]$ with values in suitable spaces of functions defined on $\Omega$. By applying the integral equations (11) and (12), we consider the following variational formulation of problem (6)-(10) in the spaces of vector-valued distributions: find $\boldsymbol{u} \in C([0, T] ; \boldsymbol{V}(\Omega)), \boldsymbol{u}^{\prime} \in L^{\infty}(0, T ; \boldsymbol{V}(\Omega)), \boldsymbol{u}^{\prime \prime} \in L^{\infty}\left(0, T ; \boldsymbol{L}^{2}(\Omega)\right)$, 
$\theta \in C\left([0, T] ; V^{\theta}(\Omega)\right), \quad \theta^{\prime} \in L^{\infty}\left(0, T ; V^{\theta}(\Omega)\right), \quad \theta^{\prime \prime} \in L^{\infty}\left(0, T ; L^{2}(\Omega)\right)$, which satisfy the following equations in the sense of distributions on $(0, T)$ :

$$
\begin{array}{ll}
\left(\rho \boldsymbol{u}^{\prime \prime}, \boldsymbol{v}\right)_{\boldsymbol{L}^{2}(\Omega)}+a(\boldsymbol{u}, \boldsymbol{v})+b(\theta, \boldsymbol{v})+\tau_{1} b\left(\theta^{\prime}, \boldsymbol{v}\right) & \\
=(\boldsymbol{f}, \boldsymbol{v})_{L^{2}(\Omega)}+(\boldsymbol{g}, \boldsymbol{v})_{\boldsymbol{L}^{2}\left(\Gamma_{1}\right)} & \forall \boldsymbol{v} \in \boldsymbol{V}(\Omega), \\
\left(\varkappa \theta^{\prime}, \varphi\right)_{L^{2}(\Omega)}+\tau_{0}\left(\varkappa \theta^{\prime \prime}, \varphi\right)_{L^{2}(\Omega)}-b^{\theta}\left(\theta^{\prime}, \varphi\right)+a^{\theta}(\theta, \varphi) & \\
+b^{\theta}\left(\varphi, \theta^{\prime}\right)-\Theta_{0} b\left(\varphi, \boldsymbol{u}^{\prime}\right)=\left(f^{\theta}, \varphi\right)_{L^{2}(\Omega)}-\left(g^{\theta}, \varphi\right)_{L^{2}\left(\Gamma_{1}^{\theta}\right)} & \forall \varphi \in V^{\theta}(\Omega),
\end{array}
$$

together with the initial conditions

$$
\boldsymbol{u}(0)=\boldsymbol{u}_{0}, \quad \boldsymbol{u}^{\prime}(0)=\boldsymbol{u}_{1}, \quad \theta(0)=\theta_{0}, \quad \theta^{\prime}(0)=\theta_{1},
$$

where

$$
\begin{aligned}
a(\hat{\boldsymbol{v}}, \boldsymbol{v}) & =\int_{\Omega} \sum_{i, j, p, q=1}^{3} \mu_{i j p q} e_{p q}(\hat{\boldsymbol{v}}) e_{i j}(\boldsymbol{v}) \mathrm{d} x \quad \forall \boldsymbol{v}, \hat{\boldsymbol{v}} \in \boldsymbol{H}^{1}(\Omega), \\
a^{\theta}(\hat{\varphi}, \varphi) & =\int_{\Omega} \sum_{p, q=1}^{3} \lambda_{p q} \frac{\partial \hat{\varphi}}{\partial x_{q}} \frac{\partial \varphi}{\partial x_{p}} \mathrm{~d} x \quad \forall \varphi, \hat{\varphi} \in H^{1}(\Omega), \\
b(\tilde{\varphi}, \boldsymbol{v}) & =\int_{\Omega} \sum_{i, j=1}^{3} \eta_{i j} \tilde{\varphi} e_{i j}(\boldsymbol{v}) \mathrm{d} x, \\
b^{\theta}(\varphi, \tilde{\varphi}) & =\int_{\Omega} \sum_{p=1}^{3} \beta_{p} \frac{\partial \varphi}{\partial x_{p}} \tilde{\varphi} \mathrm{d} x \quad \forall \tilde{\varphi} \in L^{2}(\Omega), \varphi \in H^{1}(\Omega), \boldsymbol{v} \in \boldsymbol{H}^{1}(\Omega) .
\end{aligned}
$$

Note that since $\boldsymbol{u}$ and $\theta$ are continuous with respect to the time variable $t$, the equations in (17) for $\boldsymbol{u}(0)$ and $\theta(0)$ are understood in the sense of the spaces $\boldsymbol{V}(\Omega)$ and $V^{\theta}(\Omega)$, respectively. From the embedding theorem [Dautray and Lions 1992] it follows that $\boldsymbol{u}^{\prime} \in C\left([0, T] ; \boldsymbol{L}^{2}(\Omega)\right), \theta^{\prime} \in C\left([0, T] ; L^{2}(\Omega)\right)$, and, consequently, the equations in (17) for $\boldsymbol{u}^{\prime}(0)$ and $\theta^{\prime}(0)$ are understood in the sense of the spaces $\boldsymbol{L}^{2}(\Omega)$ and $L^{2}(\Omega)$, respectively.

For the problem (15)-(17), which is equivalent to the initial-boundary value problem (6)-(10) in the spaces of classical smooth enough functions, the following existence, uniqueness, and continuous dependence theorem is valid.

Theorem 2.1. Suppose that parameters characterizing thermal and elastic properties of thermoelastic body satisfy conditions (1)-(5), $\tau_{1}>0, \tau_{0}>0$ and

$$
\rho(x)>c_{\rho}=\text { const }>0, \quad \varkappa(x)>c_{\varkappa}=\text { const }>0 \quad \forall x \in \Omega,
$$


and $\mu_{i j p q}, \lambda_{p q}, \eta_{i j}, \beta_{p} \in C^{0,1}(\bar{\Omega})(i, j, p, q=1,2,3), \rho, \varkappa \in L^{\infty}(\Omega)$. If the densities of body and surface forces, heat source, and heat flux are such that

$$
\begin{aligned}
\boldsymbol{f}, \boldsymbol{f}^{\prime}, \boldsymbol{f}^{\prime \prime} & \in L^{2}\left(0, T ; \boldsymbol{L}^{6 / 5}(\Omega)\right), \quad \boldsymbol{g}, \boldsymbol{g}^{\prime}, \boldsymbol{g}^{\prime \prime} \in L^{2}\left(0, T ; \boldsymbol{L}^{4 / 3}\left(\Gamma_{1}\right)\right), \\
f^{\theta}, f^{\theta \prime}, f^{\theta \prime \prime} & \in L^{2}\left(0, T ; L^{6 / 5}(\Omega)\right), \quad g^{\theta}, g^{\theta \prime}, g^{\theta \prime \prime} \in L^{2}\left(0, T ; L^{4 / 3}\left(\Gamma_{1}^{\theta}\right)\right),
\end{aligned}
$$

and initial conditions $\boldsymbol{u}_{0} \in \boldsymbol{H}^{2}(\Omega) \cap \boldsymbol{V}(\Omega), \boldsymbol{u}_{1} \in \boldsymbol{V}(\Omega), \theta_{0} \in H^{2}(\Omega) \cap V^{\theta}(\Omega)$, $\theta_{1} \in V^{\theta}(\Omega)$ satisfy the following compatibility conditions:

$$
\begin{aligned}
& g_{i}(0)=\left.\sum_{j=1}^{3}\left(\sum_{p, q=1}^{3} \mu_{i j p q} e_{p q}\left(\boldsymbol{u}_{0}\right)+\eta_{i j} \theta_{0}+\eta_{i j} \tau_{1} \theta_{1}\right) \nu_{j}\right|_{\Gamma_{1}}, \\
& g^{\theta}(0)=-\left.\sum_{p=1}^{3}\left(\sum_{q=1}^{3}\left(\lambda_{p q} \frac{\partial \theta_{0}}{\partial x_{q}}+\beta_{p} \theta_{1}\right) v_{p}\right)\right|_{\Gamma_{1}^{\theta}},
\end{aligned}
$$

where $i=1,2,3$, then the initial-boundary value problem (15)-(17) possesses a unique solution, which continuously depends on the given data, i.e., the mapping $\left(\boldsymbol{u}_{0}, \boldsymbol{u}_{1}, \theta_{0}, \theta_{1}, \boldsymbol{f}, \boldsymbol{f}^{\prime}, \boldsymbol{g}, \boldsymbol{g}^{\prime}, f^{\theta}, f^{\theta^{\prime}}, g^{\theta}, g^{\theta^{\prime}}\right) \rightarrow\left(\boldsymbol{u}, \boldsymbol{u}^{\prime}, \theta, \theta^{\prime}\right)$ is linear and continuous from space

$$
\begin{aligned}
\boldsymbol{V}(\Omega) \times & \boldsymbol{L}^{2}(\Omega) \times V^{\theta}(\Omega) \times L^{2}(\Omega) \times L^{2}\left(0, T ; \boldsymbol{L}^{6 / 5}(\Omega)\right) \times L^{2}\left(0, T ; \boldsymbol{L}^{6 / 5}(\Omega)\right) \\
& \times L^{2}\left(0, T ; \boldsymbol{L}^{4 / 3}\left(\Gamma_{1}\right)\right) \times L^{2}\left(0, T ; \boldsymbol{L}^{4 / 3}\left(\Gamma_{1}\right)\right) \times L^{2}\left(0, T ; L^{6 / 5}(\Omega)\right) \\
& \times L^{2}\left(0, T ; L^{6 / 5}(\Omega)\right) \times L^{2}\left(0, T ; L^{4 / 3}\left(\Gamma_{1}^{\theta}\right)\right) \times L^{2}\left(0, T ; L^{4 / 3}\left(\Gamma_{1}^{\theta}\right)\right)
\end{aligned}
$$

to space

$$
C([0, T] ; \boldsymbol{V}(\Omega)) \times C\left([0, T] ; \boldsymbol{L}^{2}(\Omega)\right) \times C\left([0, T] ; V^{\theta}(\Omega)\right) \times C\left([0, T] ; L^{2}(\Omega)\right) .
$$

Further details and extensions will be presented in [Avalishvili et al. 2017].

\section{Conclusions}

We studied an initial-boundary value problem with general mixed boundary conditions for displacement and temperature corresponding to the Green-Lindsay linear dynamical three-dimensional model for an inhomogeneous anisotropic thermoelastic body. We obtained a variational formulation of the three-dimensional problem in the corresponding spaces of vector-valued distributions with respect to the time variable with values in Sobolev spaces, which is equivalent to the original differential formulation in spaces of sufficiently smooth functions. We formulated a new theorem on the existence and uniqueness of the solution of the three-dimensional initial-boundary value problem, and the continuous dependence of the solution on given data in suitable function spaces. 


\section{References}

[Abdallah 2009] I. A. Abdallah, "Dual phase lag heat conduction and thermoelastic properties of a semi-infinite medium induced by ultrashort pulsed laser", Progress Phys. 5:3 (2009), 60-63.

[Ackerman and Overton 1969] C. C. Ackerman and W. C. Overton, Jr., "Second sound in solid helium-3", Phys. Rev. Lett. 22:15 (1969), 764-766.

[Afrin et al. 2011] N. Afrin, Y. Zhang, and J. K. Chen, “Thermal lagging in living biological tissue based on nonequilibrium heat transfer between tissue, arterial and venous bloods", Int. J. Heat Mass Transfer 54:11-12 (2011), 2419-2426.

[Avalishvili et al. 2010] G. Avalishvili, M. Avalishvili, and D. Gordeziani, "On some nonclassical two-dimensional models for thermoelastic plates with variable thickness", Bull. Georgian Natl. Acad. Sci. (N.S.) 4:2 (2010), 27-34.

[Avalishvili et al. 2017] G. Avalishvili, M. Avalishvili, and W. H. Müller, "An investigation of the Green-Lindsay three-dimensional model”, 2017. To appear in Math. Mech. Solids.

[Bargmann et al. 2008] S. Bargmann, R. Greve, and P. Steinmann, "Simulation of cryovolcanism on Saturn's moon Enceladus with the Green-Naghdi theory of thermoelasticity", Bull. Glaciol. Res. 26 (2008), 23-32.

[Bem 1983] Z. Bem, "Existence of a generalized solution in thermoelasticity with two relaxation times, II”, J. Thermal Stresses 6:2-4 (1983), 281-299.

[Burchuladze and Gegelia 1985] T. V. Burchuladze and T. G. Gegelia, Развитие метода потенциала в теории упругости, Trudy Tbiliss. Mat. Inst. Razmadze Akad. Nauk Gruzin. SSR 79, Mecniereba, Tbilisi, 1985.

[Carbonaro and Ignaczak 1987] B. Carbonaro and J. Ignaczak, "Some theorems in temperature-ratedependent thermoelasticity for unbounded domains", J. Thermal Stresses 10:3 (1987), 193-220.

[Caviglia et al. 1992] G. Caviglia, A. Morro, and B. Straughan, "Thermoelasticity at cryogenic temperatures", Internat. J. Non-Linear Mech. 27:2 (1992), 251-263.

[Chandrasekharaiah 1986] D. S. Chandrasekharaiah, "Thermoelasticity with second sound: a review", Appl. Mech. Rev. 39:3 (1986), 355-376.

[Chandrasekharaiah 1998] D. S. Chandrasekharaiah, "Hyperbolic thermoelasticity: a review of recent literature", Appl. Mech. Rev. 51:12 (1998), 705-729.

[Coleman and Newman 1988] B. D. Coleman and D. C. Newman, "Implications of a nonlinearity in the theory of second sound in solids", Phys. Rev. B 37:4 (1988), 1492-1498.

[Dautray and Lions 1992] R. Dautray and J.-L. Lions, Mathematical analysis and numerical methods for science and technology, V: Evolution problems, I, Springer, Berlin, 1992.

[Dolak and Hillen 2003] Y. Dolak and T. Hillen, "Cattaneo models for chemosensitive movement: numerical solution and pattern formation”, J. Math. Biol. 46:2 (2003), 153-170.

[Dreyer and Struchtrup 1993] W. Dreyer and H. Struchtrup, "Heat pulse experiments revisited", Contin. Mech. Thermodyn. 5:1 (1993), 3-50.

[Green 1972] A. E. Green, “A note on linear thermoelasticity”, Mathematika 19:1 (1972), 69-75.

[Green and Laws 1972] A. E. Green and N. Laws, "On the entropy production inequality", Arch. Rational Mech. Anal. 45:1 (1972), 47-53.

[Green and Lindsay 1972] A. E. Green and K. A. Lindsay, "Thermoelasticity", J. Elasticity 2:1 (1972), 1-7.

[Hetnarski and Ignaczak 2000] R. B. Hetnarski and J. Ignaczak, "Nonclassical dynamical thermoelasticity”, Int. J. Solids Struct. 37:1-2 (2000), 215-224. 
[Ignaczak and Ostoja-Starzewski 2010] J. Ignaczak and M. Ostoja-Starzewski, Thermoelasticity with finite wave speeds, Oxford Univ. Press, 2010.

[Joseph and Preziosi 1989] D. D. Joseph and L. Preziosi, "Heat waves", Rev. Modern Phys. 61:1 (1989), 41-73. Addendum in 62:2 (1990), 375-391.

[Karakostas and Massalas 1991] G. Karakostas and C. V. Massalas, "Some basic results on the generalized theory of linear thermoelasticity proposed by Green and Lindsay", European J. Mech. A Solids 10:2 (1991), 193-211.

[McLean 2000] W. McLean, Strongly elliptic systems and boundary integral equations, Cambridge Univ. Press, 2000.

[McNelly et al. 1970] T. F. McNelly, S. J. Rogers, D. J. Channin, R. J. Rollefson, W. M. Goubau, G. E. Schmidt, J. A. Krumhansl, and R. O. Pohl, "Heat pulses in NaF: onset of second sound", Phys. Rev. Lett. 24:3 (1970), 100-102.

[Müller 1971] I. Müller, "The coldness, a universal function in thermoelastic bodies", Arch. Rational Mech. Anal. 41 (1971), 319-332.

[Narayanamurti and Dynes 1972] V. Narayanamurti and R. C. Dynes, "Observation of second sound in bismuth", Phys. Rev. Lett. 28:22 (1972), 1461-1465.

[Saidane et al. 2005] A. Saidane, S. Aliouat, M. Benzohra, and M. Ketata, "A transmission line matrix (TLM) study of hyperbolic heat conduction in biological materials", J. Food Eng. 68:4 (2005), 491-496.

[Straughan 2011] B. Straughan, Heat waves, Applied Mathematical Sciences 177, Springer, New York, 2011.

[Vadasz et al. 2005] J. J. Vadasz, S. Govender, and P. Vadasz, "Heat transfer enhancement in nanofluids suspensions: possible mechanisms and explanations", Int. J. Heat Mass Transfer 48:13 (2005), 2673-2683.

[Wang and Xu 2002] X. Wang and X. Xu, "Thermoelastic wave in metal induced by ultrafast laser pulses", J. Thermal Stresses 25:5 (2002), 457-473.

[Zhu et al. 1999] X. Zhu, D. M. Villeneuve, A. Yu. Naumov, S. Nikumb, and P. B. Corkum, "Experimental study of drilling sub-10 $\mu \mathrm{m}$ holes in thin metal foils with femtosecond laser pulses", Appl. Surf. Sci. 152:3-4 (1999), 138-148.

Received 5 Sep 2016. Revised 12 Nov 2016. Accepted 19 Dec 2016.

GiA AVALISHVILI: gavalish@yahoo.com

Faculty of Exact and Natural Sciences, I. Javakhishvili Tbilisi State University, 3, I. Tchavtchavadze Ave., 0179 Tbilisi, Georgia

MARIAM AVALISHVILI: mavalish@yahoo.com

School of Informatics, Engineering and Mathematics, University of Georgia, 77a, M. Kostava Str., 0175 Tbilisi, Georgia

WOLFGANG H. MÜLLER: whmueller1000@gmail.com

Institute of Mechanics, LKM, Technische Universität Berlin, Einsteinufer 5, D-10587 Berlin, Germany 



\title{
LINEAR PANTOGRAPHIC SHEETS: ASYMPTOTIC MICRO-MACRO MODELS IDENTIFICATION
}

\author{
Claude Boutin, Francesco dell' Isola, \\ IVAN GIORGIO AND LUCA PLACIDI
}

\begin{abstract}
In this paper we consider linear pantographic sheets, which in their natural configuration are constituted by two orthogonal arrays of straight fibers interconnected by internal pivots. We introduce a continuous model by means of a micro-macro identification procedure based on the asymptotic homogenization method of discrete media. The rescaling of the mechanical properties and of the deformation measures is calibrated in order to comply with the specific kinematics imposed by the quasi-inextensibility of the fibers together with the large pantographic deformability. The obtained high-order continuum model shows interesting and exotic features related to its extreme anisotropy and also to the subcoercivity of its deformation energy. Some initial numerical simulations are presented, showing that the model can account for experimental uncommon phenomena occurring in pantographic sheets. The paper focuses on the precise analysis and the understanding of the effective behavior based on a well-calibration of the extension and bending phenomena arising at the local scale. In an upcoming work, the analysis will be extended to oblique arrays, some analytical solutions to proposed equations and some further applications.
\end{abstract}

\section{Introduction}

In the study of generalized (multiscale or architectured) continua, two different and complementary approaches can be identified:

(1) A phenomenological approach can be applied at the macroscopic scale: it gives a general framework and in general it is based on variational principles and methods [Mindlin 1965; Toupin 1964; Germain 1973; Gouin and Debieve 1986; Casal 1966; dell'Isola and Placidi 2011; dell'Isola et al. 2009; Lekszycki 1991]; however, using this approach it is not possible to make the influence of microstructure on macrophenomena become explicit. In other

\section{Communicated by Pierre Seppecher.}

MSC2010: 74KXX, 74QXX, 76AXX.

Keywords: pantographic structures, second gradient elasticity, woven fabrics. 
words, a model is established, but the microstructured material to which it could be applied is unknown.

(2) Another approach involves a scale change, made possible through a homogenization. This method has been developed in vast literature (see, e.g., [Boutin and Auriault 1993; Pideri and Seppecher 1997; Bensoussan et al. 2011; Boutin and Soubestre 2011; Auriault et al. 2009; Allaire 1992; Alibert and Della Corte 2015; dell'Isola et al. 2016b; 2016c]) but has not been applied too often to the case of multiscale materials, in which a great contrast in physical properties appears at microlevel (see [Soubestre and Boutin 2012; Boutin et al. 2010]). Moreover, homogenization methods treat more particular cases and therefore they are not supposed to cover all situations. However, a careful homogenization technique gives the possibility of identifying the micromorphologies that lead to the macroscopic behavior characteristic of generalized continua.

Recently the attention has been attracted by a particular class of microstructure: that which produces so-called pantographic continua [dell'Isola et al. 2015b; 2016b; 2016c; 2016d; Giorgio et al. 2016; Madeo et al. 2015]. This kind of structure is inspired by several natural examples; indeed, some biological tissues present fibers that can be modeled in a similar way (see, e.g., [Melnik and Goriely 2013; Federico and Grillo 2012; Grillo et al. 2015] for some possible applications). Their interest was initially related to the possibility of proving the existence of purely second gradient continua [Alibert et al. 2003], but subsequently their practical applicability has been proven for woven fabrics and some "ad hoc" designed metamaterials (see, e.g., [Del Vescovo and Giorgio 2014; Bîrsan et al. 2012; Eremeyev and Lebedev 2011; Altenbach et al. 2011; dell'Isola et al. 2015b; Eremeyev 2016]). On the other hand, some interesting theoretical results were presented in [Chesnais et al. 2015; Boutin et al. 2010; Boutin and Soubestre 2011]. In these papers, some suitable homogenization methods were introduced to study the dynamics of periodic beam structures and other evidence was presented concerning the need to introduce second gradient continua when a high contrast of mechanical properties is present at microlevel.

In all considered cases, directional (anisotropic) materials with a high contrast in properties between shear and extension are studied. This paper, different to what was done in [Rahali et al. 2015], considers the case of extensible fibers and gives a more solid foundation to and generalizes the heuristic results presented in [Placidi et al. 2017] based on an accurate analysis of the different and relative order of magnitude of the involved physical phenomena.

In this paper we will use a micro-macro asymptotic identification method and obtain the macroscopic equilibrium equations for pantographic lattices in the neighborhood of a reference configuration. The analysis of the obtained equation is 


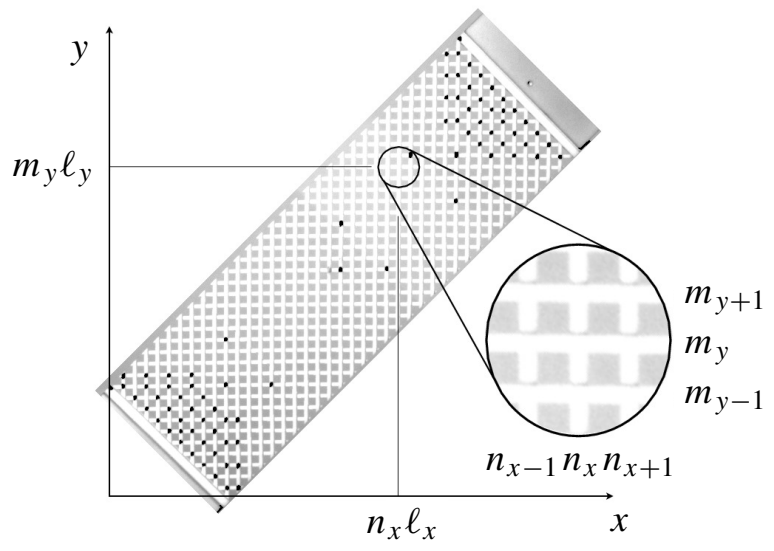

Figure 1. Topology of the pantographic lattice. The pivots are equally distributed on the whole sheet. The black points are indicating specific monitoring points during bias test experiments.

started and some equilibrium problems are solved by means of numerical simulations.

In a forthcoming paper we treat the generalized case of reference configurations constituted by two oblique arrays of straight beams, some semianalytical solutions and some further application.

\section{Hypotheses and notations}

Let us consider a periodic pantograph network of fibers (which we also call a pantographic lattice or pantographic sheet) formed of two families of continuous fibers arranged perpendicularly and along the axes $x$ and $y$. The fibers oriented along $x$ are identical, as well as those oriented along $y$. However, the two families may differ from one another. These fibers are connected by perfect pivots, with an axis perpendicular to the $\{x, y\}$ plane. The fibers oriented along $x$ are spaced periodically by the length $\ell_{y}$. Those oriented along $y$ are spaced by $\ell_{x}$. This defines the rectangular mesh, which is constituted by those two elements that are the portions of the orthogonal fibers that cross a pivot. Each pivot is referenced by two integers $n_{x}$ and $m_{y}$, which are simply its discrete coordinates along the axes $x$ and $y$, respectively. We will model each segment of fiber between two consequent nodes as a beam. The four beam elements connected to the pivot $\left\{n_{x}, m_{y}\right\}$ are denoted by $\left[n_{x}-1, n_{x}\right]$ and $\left[n_{x}, n_{x}+1\right]$ for the two elements oriented along $x$, or by $\left[m_{y}-1, m_{y}\right]$ and $\left[m_{y}, m_{y}+1\right]$ for the two elements oriented along $y$. The understanding of the reader is helped by Figure 1.

The parameters of the beam elements are their Young's modulus $E_{j}$, the area $A_{j}$ of their sections and the moments of inertia $I_{j}$ of their sections, where $j=x, y$. 
The dimensions of the sections $\sqrt{A_{j}}$ are assumed small with respect to the lengths $\ell_{j}$. Accordingly, the behavior of the interpivot elements can be effectively modeled by the Euler beam model. It is further assumed that the geometrical and the mechanical parameters of both types of beams are of the same order of magnitude: to be more precise, the period is characterized by the length $\ell=\sqrt{\ell_{x} \ell_{y}}$ in such a way that $O\left(\ell_{x}\right)=O\left(\ell_{y}\right)=\ell$. We also introduce the following dimensionless quantities: $\ell_{x}^{\star}=\ell_{x} / \ell$ and $\ell_{y}^{\star}=\ell_{y} / \ell$ so that $\ell_{x}^{\star} \ell_{y}^{\star}=1$ and $O\left(\ell_{x}^{\star}\right)=O\left(\ell_{y}^{\star}\right)=O(1)$.

Note that the specificity of the adopted assumption of beam behavior is that the length of the arrays is not considered (i.e., the fibers are not treated as beams) but instead, this is the topology of the pantographic lattice that leads to a local beam description.

We study in the $\{x, y\}$ plane the quasistatic small deformations of the lattice in the neighborhood of its initial equilibrium position where the lattice is periodic and has a rectangular period. It is also assumed that the spatial variations of the deformation and placement fields occur at large-scale and have a characteristic variation length $L$ that is large compared to the size of period $\ell$; in other words, to have a relevant variation of the deformation fields, the space variables must have a large increment relative to $\ell$.

These considerations naturally introduce the small parameter to be used for specifying scale separation:

$$
\varepsilon=\ell / L \ll 1 .
$$

The macroscopic description of the system, valid at the dominant order, is reached for $\varepsilon \rightarrow 0$.

\section{Homogenization method: multiscale asymptotic heuristic approach}

Let us construct the global behavior from the local behavior of beam elements connected by pivots. To do this, the problem is first discretized exactly and then it is converted into a continuum macroscopic formulation by an asymptotic homogenization procedure.

Using the balance laws of an Euler beam, the contact actions (normal and shear forces and moments) at the ends of each beam element are explicitly expressed in terms of the kinematic variables (displacements and rotations) evaluated at the same ends. The balance of each element is thereby assured. We will consider the cases in which the beam elements between the nodes deform in a quasistatic regime. In other words, our treatment will be applicable when, in considered phenomena, there is a (quasi-)instantaneous equilibrium, at the level of the periodic cell, of the interconnected beams. Sometimes this assumption is referred to as the assumption of local microscopic instantaneous equilibrium. This approach is relevant when dealing with phenomena of slow-time evolution. 
As a consequence of the exact discretization, to specify the conditions of the global equilibrium conditions, it is necessary and sufficient to focus on the equilibrium of each one of the pivots, which will play the role of material points of the homogenized continuum. It is therefore expressed, in each pivot, the balance of forces and moments applied by the four elements therein connected (belonging to the two orthogonal fibers that intersect at each pivot). This gives an exact representation of the original problem in a discrete form of finite difference type, with the variables being the kinematic variables and the actions of each element, evaluated at the pivot-locations. One has to explicitly remark that in the set of the aforementioned four elements, one can distinguish two pairs (parallel in the reference configuration) which are indeed part of the same fiber: moving from one to the other elements belonging to the same fiber, the displacements and rotations are continuous (see Figure 1, $x$-fiber and $y$-fiber).

Remark also that the action of a pivot on the two continuous fibers which the pivot itself is interconnecting is modeled here as concentrated (in a point) force and couple; of course, by the action and reaction principle, the action of the pivot on the fiber of one array is opposite to the action exerted by the same pivot on the fiber of the other array.

The passage from the exact discrete formulation to the macroscopic continuous description, valid at the dominant order, is performed as follows [Caillerie 1984]. We assume that the overall behavior of the system can be described by a set of macroscopic fields, the generic element of which is denoted by $Q(x, y)$.

Discrete variables $q_{\left(n_{x}, m_{y}\right)}$ at the pivots are considered as the values at these points of continuous functions $Q(x, y)$.

This procedure has been described in general already in the works by Piola [2014] (he seems to us to be among the first scientists having introduced such a heuristic method of homogenization; see [dell'Isola et al. 2016d; Rahali et al. 2015]); however, he applied it specifically only to the case of fluids.

We therefore set

$$
q_{\left(n_{x}, n_{y}\right)}=Q\left(x_{n}, y_{m}\right), \quad x_{n}=n_{x} \ell_{x}, \quad y_{m}=m_{y} \ell_{y} .
$$

Consistent with the hypothesis of separation of scale, these continuous functions vary at large scales, such as $O(L)$. Consequently, the increments of the interpivot distance $O(\ell)$ can be expressed by the Taylor expansions of macroscopic fields. Thus, the finite difference of the discrete formulation is converted into series by introducing successive gradients of the macroscopic functions. As the distances between the pivots are constant due to the periodicity, the terms of such series are of the type, for example, $\ell_{x}^{k} \partial^{k} Q / \partial x^{k}=O\left(\ell_{x}^{k} / L^{k}\right) O(Q)=\varepsilon^{k} O(Q)$, with a multiplication factor. They therefore involve the orders of magnitude in powers of $\varepsilon$. As we are interested in situations where $\varepsilon \rightarrow 0$, we need the following specification for 
the consistency of the orders of magnitude, i.e., we need to represent the functions $Q(x, y)$ in the form of asymptotic expansions of the type:

$$
Q(x, y)=\sum_{k=0}^{\infty} \varepsilon^{k} Q^{(k)}(x, y), \quad O\left(Q^{(k)}\right)=O\left(Q^{(0)}\right) .
$$

These asymptotic expansions are to be used in the equations of equilibrium expressed via Taylor expansions. The dimensionless parameters that emerge from this formulation must be weighted in powers of $\varepsilon$ to translate correctly the dominant mechanisms in the studied system [Boutin and Hans 2003; Hans and Boutin 2008]. This normalization of the balance equations preserves the same local physics during the transition to the limit $\varepsilon \rightarrow 0$. Consequently, the macroscopic model in the limit preserves - at the dominant order - the same local physics than that prevailing in the real system, where the scale ratio $\ell / L$ takes small but finite values.

After the aforementioned normalization, we obtain a series of equilibrium conditions in terms of the powers of $\varepsilon$, which may be solved term by term. The macroscopic description, at the dominant order, is constituted by the first nontrivial differential system on the macroscopic variables.

\section{Micromodeling of pantographic lattices}

In the literature, much attention has been paid to lattices of beams interconnected by clamping constraints and to trusses [Németh and Kocsis 2014; Liew et al. 2000; Noor et al. 1978]. However, the existence of so called "floppy modes" at the microlevel in the pantographic structures requires the most attentive consideration. To be more precise: what we call a floppy mode is a deformation of the microstructure to which is associated a vanishing energy (for more details, see [Alibert et al. 2003; Seppecher et al. 2011]). An intuitive consequence of the existence of floppy modes is that for the effective medium, the standard condition of coerciveness is not verified and therefore this concept needs to be modified or generalized. Actually one can decompose the space of deformations into a coercive subspace plus the space of floppy modes and the role of the boundary condition becomes more determinant in well-posed problems.

We used these microscopic floppy modes to prove that, in general, the class of first-gradient continua (those introduced by Cauchy and usually considered in continuum mechanics; see, e.g., [dell'Isola et al. 2016a; 2015c]) is not enough to describe, at a macrolevel, all conceivable physical systems. In particular, when there is high contrast (see [Camar-Eddine and Seppecher 2001; Pideri and Seppecher 1997; Hans and Boutin 2008; Boutin and Soubestre 2011; Cecchi and Rizzi 2001]) in physical properties at the microlevel, it may become necessary at the macrolevel to introduce higher-order continua (see [dell'Isola et al. 2012; 2016e]). 
The analysis which we present adapts the studies presented in [Boutin et al. 2010; Boutin and Soubestre 2011; Hans and Boutin 2008; Soubestre and Boutin 2012] to the case of pantographic lattices to take into account their behavior, which can be regarded as being somehow exotic.

We start by using the framework of local microscopic instantaneous equilibrium to formulate a mesolevel model where the lattice is described as a set of nodes (the pivots) interconnected by beam element.

4.1. Characterization of the mechanical behavior of a beam element. Let the section of the considered straight (in the reference configuration) beam be $A$, its moment of inertia being $I$, and let the material constituting it be elastic, isotropic and with Young's modulus $E$. We denote (referring to the beam planar reference configuration) by $v$ the axial displacement, by $w$ the transverse displacement, by $N$ the normal force, by $T$ the shear force and by $M$ the bending moment (we will be using the French convention for the orientation of axes). These contact actions are defined as that of the part $s<0$ on part $s>0$, where $s$ designates the abscissa along the axis of the beam. Consider a portion of the beam between two points $B$ and $C$, spaced apart by a distance $\ell$ that is large enough compared to the size $\sqrt{A}$ of the beam section. The Euler beam theory can therefore be used. Accordingly, the rotation of the section is related to the transverse displacement via the relation $\theta(s)=\mathrm{d} w(s) / \mathrm{d} s$. The constitutive equations of the beam are the following:

$$
N(s)=E A \frac{\mathrm{d} v}{\mathrm{~d} s}, \quad M(s)=-E I \frac{\mathrm{d}^{2} w}{\mathrm{~d} s^{2}},
$$

and, in the quasistatic regime, the equilibrium equations in differential form are expressed by

$$
\frac{\mathrm{d} N}{\mathrm{~d} s}=0, \quad \frac{\mathrm{d} T}{\mathrm{~d} s}=0, \quad \frac{\mathrm{d} M}{\mathrm{~d} s}+T=0 .
$$

Suppose that for the point $B$ (and $C$ ), the displacements and the rotation are $v^{B}$, $w^{B}$ and $\theta^{B}$ (respectively $v^{C}, w^{C}$ and $\theta^{C}$ ). The forces and moment at $B$ and at $C$ are determined using the equations of beams. They are expressed as a function of the kinematic variables as follows:

$$
\begin{aligned}
N^{B}=N^{C} & =\frac{E A}{\ell}\left(v^{B}-v^{C}\right), \\
T^{B}=T^{C} & =\frac{12 E I}{\ell^{3}}\left(w^{B}-w^{C}+\frac{1}{2} \ell\left(\theta^{B}+\theta^{C}\right)\right), \\
M^{B} & =\frac{6 E I}{\ell^{2}}\left(w^{B}-w^{C}+\frac{1}{3} \ell\left(2 \theta^{B}+\theta^{C}\right)\right), \\
M^{C}= & -\frac{6 E I}{\ell^{2}}\left(w^{B}-w^{C}+\frac{1}{3} \ell\left(\theta^{B}+2 \theta^{C}\right)\right) .
\end{aligned}
$$


Expressions of $N$ involve the axial $E A / \ell$ rigidities, while $T$ and $M$ involve bending $12 E I / \ell^{3}$ rigidities. The beam's slenderness hypothesis $\sqrt{A} \ll \ell$ implies that such rigidities differ significantly. In fact, considering beams of regular section (for instance, the rectangular section of length's sides $a$ and $b$, with $b=O(a)=O(\sqrt{A})$, then $A=a b, I=b a^{3} / 12$ and $12 I / A=a^{2}=O(A)$, and consequently the rigidity ratio $R$ is

$$
R=\frac{12 E I}{\ell^{3}} \frac{\ell}{E A}=\frac{12 I}{\ell^{2} A}=O\left(\frac{A}{\ell^{2}}\right) \ll 1 .
$$

This strong stiffness contrast plays an essential role in the functioning of the system. Hereafter we take into account explicitly that the aspect ratio of the elements is $\sqrt{A} / \ell=O(\varepsilon)$ which leads to

$$
R=\frac{12 E I}{\ell^{3}} \frac{\ell}{E A}=O\left(\varepsilon^{2}\right) .
$$

4.2. Discrete kinematic variables and equilibrium at pivots. Because of the operating principle of an internal pivot, the ends of the four elements connected to it undergo the same displacement $u_{x}\left(u_{y}\right)$ along the axis $x(y)$, but rotations of those elements belonging to fibers with distinct orientations are not identical; the coinciding ends of the two elements oriented along $x(y)$ undergo the same rotation $\theta_{x}$ $\left(\theta_{y}\right)$. Thus, each pivot $\left\{n_{x}, m_{y}\right\}$ is described by four kinematic variables $u_{x\left(n_{x}, m_{y}\right)}$, $u_{y\left(n_{x}, m_{y}\right)}, \theta_{x\left(n_{x}, m_{y}\right)}$ and $\theta_{y\left(n_{x}, m_{y}\right)}$.

The equilibrium at a pivot results:

(i) in the balances of force (exerted on the pivot) along $x$ and $y$, and

(ii) in the continuity of both moment fields arising in the beam elements oriented either along $x$ or along $y$.

These four equations expressed at the pivot $\left\{n_{x}, m_{y}\right\}$ take the following forms:

- balance of force along $x$ :

$$
-T_{\left[m_{y}-1, m_{y}\right]}^{C}+T_{\left[m_{y}, m_{y}+1\right]}^{B}+N_{\left[n_{x}-1, n_{x}\right]}^{C}-N_{\left[n_{x}, n_{x}+1\right]}^{B}=0,
$$

- balance of force along $y$ :

$$
T_{\left[n_{x}-1, n_{x}\right]}^{C}-T_{\left[n_{x}, n_{x}+1\right]}^{B}+N_{\left[m_{y}-1, m_{y}\right]}^{C}-N_{\left[m_{y}, m_{y}+1\right]}^{B}=0,
$$

- balance of moments for the elements along $x$ :

$$
M_{\left[n_{x}-1, n_{x}\right]}^{C}-M_{\left[n_{x}, n_{x}+1\right]}^{B}=0,
$$

- balance of moments for the elements along $y$ :

$$
M_{\left[m_{y}-1, m_{y}\right]}^{C}-M_{\left[m_{y}, m_{y}+1\right]}^{B}=0 .
$$


Here, we have denoted by the symbol $Q_{[p, q]}^{D}$ the value of the field $Q$ at the extremity $D=C$ or $D=B$ of the beam element connecting the node $p$ and the node $q$, where $p$ and $q$ are consecutive in either the $x$ or $y$ direction.

The component $u_{x}\left(u_{y}\right)$ of the pivot displacement is:

(i) the axial displacement of the ends of the beam element oriented along $x(y)$, and

(ii) the opposite transverse (direct) displacement of the ends of the beam element along $y$ (along $x$ ). The change of sign results from different orientations of the global frame and of the local frame of the $y$-oriented fibers.

Thus, by substituting in (9)-(12) the forces by their expressions in terms of the displacement fields (3)-(6), the balance of force along $x$ is obtained as

$$
\begin{aligned}
\frac{12 E_{y} I_{y}}{\ell_{y}^{3}}\left(\left(u_{x\left(n_{x}, m_{y}-1\right)}\right.\right. & \left.-2 u_{x\left(n_{x}, m_{y}\right)}+u_{x\left(n_{x}, m_{y}+1\right)}\right) \\
+ & \frac{1}{2} \ell_{y}\left(-\theta_{y\left(n_{x}, m_{y}-1\right)}+\theta_{\left.y\left(n_{x}, m_{y}+1\right)\right)}\right) \\
+ & \frac{E_{x} A_{x}}{\ell_{x}}\left(u_{x\left(n_{x}-1, m_{y}\right)}-2 u_{x\left(n_{x}, m_{y}\right)}+u_{x\left(n_{x}+1, m_{y}\right)}\right)=0 .
\end{aligned}
$$

The continuity at the nodes of bending moments of the elements oriented along $y$ (after the simplification by $2 E_{y} I_{y} / \ell_{y}$ ) reads

$$
\frac{3}{\ell_{y}}\left(u_{x\left(n_{x}, m_{y}-1\right)}-u_{x\left(n_{x}, m_{y}+1\right)}\right)-\left(\theta_{y\left(n_{x}, m_{y}-1\right)}+4 \theta_{y\left(n_{x}, m_{y}\right)}+\theta_{y\left(n_{x}, m_{y}+1\right)}\right)=0,
$$

the balance of force along $y$ reads

$$
\begin{aligned}
\frac{12 E_{x} I_{x}}{\ell_{x}^{3}}\left(\left(u_{y\left(n_{x}-1, m_{y}\right)}\right.\right. & \left.-2 u_{y\left(n_{x}, m_{y}\right)}+u_{y\left(n_{x}+1, m_{y}\right)}\right) \\
+ & \left.\frac{1}{2} \ell_{x}\left(-\theta_{x\left(n_{x}-1, m_{y}\right)}+\theta_{x\left(n_{x}+1, m_{y}\right)}\right)\right) \\
& +\frac{E_{y} A_{y}}{\ell_{y}}\left(u_{y\left(n_{x}, m_{y}-1\right)}-2 u_{y\left(n_{x}, m_{y}\right)}+u_{y\left(n_{x}, m_{y}+1\right)}\right)=0,
\end{aligned}
$$

and finally the continuity at the nodes of bending moments of the elements oriented along $x$ (after the simplification by $2 E_{x} I_{x} / \ell_{x}$ ) reads

$$
\frac{3}{\ell_{x}}\left(-u_{y\left(n_{x}-1, m_{y}\right)}+u_{y\left(n_{x}+1, m_{y}\right)}\right)-\left(\theta_{x\left(n_{x}-1, m_{y}\right)}+4 \theta_{x\left(n_{x}, m_{y}\right)}+\theta_{x\left(n_{x}+1, m_{y}\right)}\right)=0 .
$$

These four equations are split into two independent groups of equations: (13)-(14) couples the variables $u_{x}$ and $\theta_{y}$; while (15)-(16) couples the variables $u_{y}$ and $\theta_{x}$. Thus, it is sufficient to treat (13)-(14), being that the results of (15)-(16) are easily deduced by changing the roles of axes $x$ and $y$. 
4.3. Continuous formulation and asymptotic expansions. Let us introduce continuous kinematic descriptors (denoted by uppercase letters) coinciding with the discrete kinematic variables of the pivots $\left\{n_{x}, m_{y}\right\}$ with coordinates $x_{n}=n \ell_{x}$ and $y_{m}=m \ell_{y}$ :

$$
\begin{array}{ll}
u_{x\left(n_{x}, m_{y}\right)}=U_{x}\left(x_{n}, y_{m}\right), & \theta_{y\left(n_{x}, m_{y}\right)}=\Theta_{y}\left(x_{n}, y_{m}\right), \\
u_{y\left(n_{x}, m_{y}\right)}=U_{y}\left(x_{n}, y_{m}\right), & \theta_{x\left(n_{x}, m_{y}\right)}=\Theta_{x}\left(x_{n}, y_{m}\right),
\end{array}
$$

and use Taylor series expansions to express the terms of the finite difference equations (13)-(14). By introducing the dimensionless variables $x^{\star}=x / L$ and $y^{\star}=$ $y / L$, we have (for comparison, see [Piola 2014; Carcaterra et al. 2015])

$$
\begin{array}{r}
u_{x\left(n_{x}, m_{y}-1\right)-} 2 u_{x\left(n_{x}, m_{y}\right)}+u_{x\left(n_{x}, m_{y}+1\right)} \\
=\ell_{y}^{2} \frac{\partial^{2} U_{x}}{\partial y^{2}}\left(x_{n}, y_{m}\right)+\frac{2}{4 !} \ell_{y}^{4} \frac{\partial^{4} U_{x}}{\partial y^{4}}\left(x_{n}, y_{m}\right)+O\left(\ell_{y}^{6} \frac{\partial^{6} U_{y}}{\partial y^{6}}\right) \\
=\varepsilon^{2} \ell_{y}^{\star 2} \frac{\partial^{2} U_{x}}{\partial y^{\star 2}}+\varepsilon^{4} \ell_{y}^{\star 4} \frac{2}{4 !} \frac{\partial^{4} U_{x}}{\partial y^{\star 4}}+\varepsilon^{6} \ell_{y}^{\star 6} \frac{2}{6 !} \frac{\partial^{6} U_{x}}{\partial y^{\star 6}}+O\left(\varepsilon^{8}\right), \\
u_{x\left(n_{x}-1, m_{y}\right)}-2 u_{x\left(n_{x}, m_{y}\right)}+u_{x\left(n_{x}+1, m_{y}\right)} \\
=\varepsilon^{2} \ell_{x}^{\star 2} \frac{\partial^{2} U_{x}}{\partial x^{\star 2}}+\varepsilon^{4} \ell_{x}^{\star 4} \frac{2}{4 !} \frac{\partial^{4} U_{x}}{\partial x^{\star 4}}+\varepsilon^{6} \ell_{x}^{\star 6} \frac{2}{6 !} \frac{\partial^{6} U_{x}}{\partial x^{\star 6}}+O\left(\varepsilon^{8}\right),
\end{array}
$$

similarly,

$$
\begin{aligned}
& -\theta_{y\left(n_{x}, m_{y}-1\right)}+\theta_{y\left(n_{x}, m_{y}+1\right)} \\
& =2 \varepsilon \ell_{y}^{\star} \frac{\partial \Theta_{y}}{\partial y^{\star}}+\varepsilon^{3} \ell_{y}^{\star 3} \frac{2}{3 !} \frac{\partial^{3} \Theta_{y}}{\partial y^{\star 3}}+\varepsilon^{5} \ell_{y}^{\star 5} \frac{2}{5 !} \frac{\partial^{5} \Theta_{y}}{\partial y^{\star 5}}+O\left(\varepsilon^{7}\right), \\
& -u_{x\left(n_{x}, m_{y}-1\right)}+u_{x\left(n_{x}, m_{y}+1\right)} \\
& =2 \varepsilon \ell_{y}^{\star} \frac{\partial U_{x}}{\partial y^{\star}}+\varepsilon^{3} \ell_{y}^{\star 3} \frac{2}{3 !} \frac{\partial^{3} U_{x}}{\partial y^{\star 3}}+\varepsilon^{5} \ell_{y}^{\star 5} \frac{2}{5 !} \frac{\partial^{5} u_{x}}{\partial y^{\star 5}}+O\left(\varepsilon^{7}\right),
\end{aligned}
$$

and finally,

$$
\begin{aligned}
\theta_{y\left(n_{x}, m_{y}-1\right)} & +4 \theta_{y\left(n_{x}, m_{y}\right)}+\theta_{y\left(n_{x}, m_{y}+1\right)} \\
& =6 \Theta_{y}+\varepsilon^{2} \ell_{y}^{\star 2} \frac{\partial^{2} \Theta_{y}}{\partial y^{\star 2}}+\varepsilon^{4} \ell_{y}^{\star 4} \frac{2}{4 !} \frac{\partial^{4} \Theta_{y}}{\partial y^{\star 4}}+\varepsilon^{6} \ell_{y}^{\star 6} \frac{2}{6 !} \frac{\partial^{6} \Theta_{y}}{\partial y^{\star 6}}+O\left(\varepsilon^{8}\right) .
\end{aligned}
$$

By construction, the coefficients of the power expansions for $\varepsilon$ in (20)-(23) are of the same dominant order, but also they contain terms of lower order. Therefore, to really order the relative weight of the different addends, it is necessary to introduce the asymptotic expansions of the variables $U_{x}, U_{y}, \Theta_{x}$ and $\Theta_{y}$. This is essential to effectively separate the power exponents and to ensure the coherence of the passage to the limit $\varepsilon \rightarrow 0$. It should be noted that consecutive terms of Taylor 
expansions are systematically offset from $\varepsilon^{2}$. It is therefore sufficient to introduce the developments in the even powers of $\varepsilon$. Consequently, we are looking for fields $U_{x}, U_{y}, \Theta_{x}, \Theta_{y}$ in the generic form

$$
U_{x}=U_{x}^{(0)}+\varepsilon^{2} U_{x}^{(2)}+\varepsilon^{4} U_{x}^{(4)}+O\left(\varepsilon^{6} U_{x}^{(6)}\right) .
$$

Thereafter, we will denote with a tilde the correction terms that are physically observable, e.g.,

$$
\widetilde{U}_{x}^{(4)}=\varepsilon^{4} U_{x}^{(4)}, \quad \widetilde{\Theta}_{x}^{(4)}=\varepsilon^{4} \Theta_{x}^{(4)} .
$$

Referring the developments in power of $\varepsilon^{2}$ in (20)-(23), we get

$$
\begin{aligned}
& u_{x\left(n_{x}, m_{y}-1\right)}-2 u_{x\left(n_{x}, m_{y}\right)}+u_{x\left(n_{x}, m_{y}+1\right)} \\
& =\varepsilon^{2} \ell_{y}^{\star 2} \frac{\partial^{2} U_{x}^{(0)}}{\partial y^{\star 2}}+\varepsilon^{4} \ell_{y}^{\star 4}\left(\frac{\partial^{2} U_{x}^{(2)}}{\partial y^{\star 2}}+\frac{2}{4 !} \frac{\partial^{4} U_{x}^{(0)}}{\partial y^{\star 4}}\right)+O\left(\varepsilon^{6}\right), \\
& u_{x\left(n_{x}-1, m_{y}\right)}-2 u_{x\left(n_{x}, m_{y}\right)}+u_{x\left(n_{x}+1, m_{y}\right)} \\
& =\varepsilon^{2} \ell_{x}^{\star 2} \frac{\partial^{2} U_{x}^{(0)}}{\partial x^{\star 2}}+\varepsilon^{4} \ell_{x}^{\star 4}\left(\frac{\partial^{2} U_{x}^{(2)}}{\partial x^{\star 2}}+\frac{2}{4 !} \frac{\partial^{4} U_{y}^{(0)}}{\partial x^{\star 4}}\right)+O\left(\varepsilon^{6}\right), \\
& -\theta_{y\left(n_{x}, m_{y}-1\right)}+\theta_{y\left(n_{x}, m_{y}+1\right)} \\
& =\varepsilon \ell_{y}^{\star} 2 \frac{\partial \Theta_{y}^{(0)}}{\partial y^{\star}}+\varepsilon^{3} \ell_{y}^{\star 3}\left(2 \frac{\partial \Theta_{y}^{(2)}}{\partial y^{\star}}+\frac{2}{3 !} \frac{\partial^{3} \Theta_{y}^{(0)}}{\partial y^{\star 3}}\right) \\
& +\varepsilon^{5} \ell_{y}^{\star 5}\left(2 \frac{\partial \Theta_{y}^{(4)}}{\partial y^{\star}}+\frac{2}{3 !} \frac{\partial^{3} \Theta_{y}^{(0)}}{\partial y^{\star 3}}+\frac{2}{5 !} \frac{\partial^{5} \Theta_{y}^{(0)}}{\partial y^{\star 5}}\right)+O\left(\varepsilon^{7}\right), \\
& -u_{x\left(n_{x}, m_{y}-1\right)}+u_{x\left(n_{x}, m_{y}+1\right)} \\
& =\varepsilon \ell_{y}^{\star} 2 \frac{\partial U_{x}^{(0)}}{\partial y^{\star}}+\varepsilon^{3} \ell_{y}^{\star 3}\left(2 \frac{\partial U_{x}^{(2)}}{\partial y^{\star}}+\frac{2}{3 !} \frac{\partial^{3} U_{x}^{(0)}}{\partial y^{\star 3}}\right) \\
& +\varepsilon^{5} \ell_{y}^{\star 5}\left(2 \frac{\partial U_{x}^{(4)}}{\partial y^{\star}}+\frac{2}{3 !} \frac{\partial^{3} U_{x}^{(2)}}{\partial y^{\star 3}}+\frac{2}{5 !} \frac{\partial^{5} U_{x}^{(0)}}{\partial y^{\star 5}}\right)+O\left(\varepsilon^{7}\right), \\
& \theta_{y\left(n_{x}, m_{y}-1\right)}+4 \theta_{y\left(n_{x}, m_{y}\right)}+\theta_{y\left(n_{x}, m_{y}-1\right)} \\
& =6 \Theta_{y}^{(0)}+\varepsilon^{2} \ell_{y}^{\star 2}\left(\Theta_{y}^{(2)}+\frac{\partial^{2} \Theta_{y}^{(0)}}{\partial y^{\star 2}}\right) \\
& +\varepsilon^{4} \ell_{y}^{\star 4}\left(\Theta_{y}^{(4)}+\frac{\partial^{2} \Theta_{y}^{(2)}}{\partial y^{\star 2}}+\frac{2}{4 !} \frac{\partial^{4} \Theta_{y}^{(0)}}{\partial y^{\star 4}}\right)+O\left(\varepsilon^{6}\right) .
\end{aligned}
$$


The calculations presented in this subsection provides an accurate transformation of the finite differences into successive derivatives. This step is essential to get the continuous asymptotic model valid in the limit $\varepsilon \rightarrow 0$.

\section{Asymptotic macroscopic model}

In (24)-(28), the macroscopic continuous fields and their macroscopic derivatives appear. By substituting them in the equilibrium equations (13)-(14) we may obtain a macroscopic continuous formulation of the equilibrium of pivots (equilibrium of force along $x$ and equilibrium of moment for the elements along $y$ ). To make explicit which are the appearing powers of $\varepsilon$, it is convenient to write the obtained equations in the nondimensional variables $x^{\star}$ and $y^{\star}$, where $L$ is the reference length. As, by hypothesis, $\ell_{x}$ and $\ell_{y}$ are of order $\varepsilon$ with respect to $L$, we have $\ell_{x}=\ell_{x}^{\star} \ell=\varepsilon \ell_{x}^{\star} L$ and $\ell_{y}=\varepsilon \ell_{y}^{\star} L$. Thus, by limiting ourselves to the infinitesimals $O\left(\varepsilon^{6}\right)$, the continuity of moments of the elements oriented along $y$ (14) gives, after grouping different terms,

$$
\begin{aligned}
& 6\left(\frac{\partial U_{x}^{(0)}}{\partial y^{\star}}+L \ell_{y}^{\star} \Theta_{y}^{(0)}\right)+\varepsilon^{2} \ell_{y}^{\star 2} 6\left(\frac{\partial U_{x}^{(2)}}{\partial y^{\star}}+L \ell_{y}^{\star} \Theta_{y}^{(2)}\right) \\
& +\varepsilon^{4} \ell_{y}^{\star 4}\left(6\left(\frac{\partial U_{x}^{(4)}}{\partial y^{\star}}+L \ell_{y}^{\star} \Theta_{y}^{(4)}\right)+\frac{2}{4 !} \frac{\partial^{4}}{\partial y^{\star 4}}\left(\frac{1}{5} \frac{\partial U_{x}^{(0)}}{\partial y^{\star}}+L \ell_{y}^{\star} \Theta_{y}^{(0)}\right)\right)+O\left(\varepsilon^{6}\right)=0,
\end{aligned}
$$

and the equilibrium of forces along $x$ (13) gives

$$
\begin{aligned}
& R_{x} \varepsilon^{2} \ell_{y}^{\star 2}\left(\frac{\partial}{\partial y^{\star}}\left(\frac{\partial U_{x}^{(0)}}{\partial y^{\star}}+L \ell_{y}^{\star} \Theta_{y}^{(0)}\right)\right. \\
& \left.+\varepsilon^{2} \ell_{y}^{\star 2}\left\{\frac{\partial}{\partial y^{\star}}\left(\frac{\partial U_{x}^{(2)}}{\partial y^{\star}}+L \ell_{y}^{\star} \Theta_{y}^{(2)}\right)+\frac{\partial^{3}}{\partial y^{\star 3}}\left(\frac{2}{4 !} \frac{\partial U_{x}^{(0)}}{\partial y^{\star}}+\frac{1}{3 !} L \ell_{y}^{\star} \Theta_{y}^{(0)}\right)\right\}+O\left(\varepsilon^{4}\right)\right) \\
& +\varepsilon^{2} \ell_{x}^{\star 2}\left(\frac{\partial^{2} U_{x}^{(0)}}{\partial x^{\star 2}}+\varepsilon^{2} \ell_{x}^{\star 2}\left\{\frac{\partial^{2} U_{x}^{(2)}}{\partial x^{\star 2}}+\frac{2}{4 !} \frac{\partial^{4} U_{x}^{(0)}}{\partial x^{\star 4}}\right\}\right. \\
& \left.+\varepsilon^{4} \ell_{x}^{\star 4}\left\{\frac{\partial^{2} U_{x}^{(4)}}{\partial x^{\star 2}}+\frac{2}{4 !} \frac{\partial^{4} U_{x}^{(2)}}{\partial x^{\star 4}}+\frac{2}{6 !} \frac{\partial^{6} U_{x}^{(0)}}{\partial x^{\star 6}}\right\}+O\left(\varepsilon^{6}\right)\right)=0,
\end{aligned}
$$

where

$$
R_{x}=\frac{12 E_{y} I_{y}}{\ell_{y}^{3}} \frac{\ell_{x}}{E_{x} A_{x}}
$$

The moment in (29) comes in the form of a series in which the mechanical characteristics of beams do not interfere. The convergence of the series when $\varepsilon \rightarrow 0$ 
implies that each involved term vanishes. Consequently,

$$
\begin{gathered}
\frac{\partial U_{x}^{(0)}}{\partial y^{\star}}+L \ell_{y}^{\star} \Theta_{y}^{(0)}=0, \\
\frac{\partial U_{x}^{(2)}}{\partial y^{\star}}+L \ell_{y}^{\star} \Theta_{y}^{(2)}=0, \\
\frac{\partial U_{x}^{(4)}}{\partial y^{\star}}+L \ell_{y}^{\star} \Theta_{y}^{(4)}+\frac{1}{3 \cdot 4 !} \frac{\partial^{4}}{\partial y^{\star 4}}\left(\frac{1}{5} \frac{\partial U_{x}^{(0)}}{\partial y^{\star}}-L \ell_{y}^{\star} \Theta_{y}^{(0)}\right)=0 .
\end{gathered}
$$

This precisely means that the equilibrium of moments for $y$ fibers requires, with an error being equal to $O\left(\varepsilon^{4}\right)$, a relationship between their rotation and their transverse gradient, which is expressed in the dimensional fields as

$$
\frac{\partial U_{x}}{\partial y}+\ell_{y}^{\star} \Theta_{y}=O\left(\varepsilon^{4}\right) .
$$

At the dominant order, $\Theta_{y}$ can thus be considered a hidden variable which does not emerge in the macroscopic description at the leading order. Only by considering the correction $O\left(\varepsilon^{4}\right)$ does the rotation differ from the transverse gradient because we have

$$
\frac{\partial U_{x}^{(4)}}{\partial y^{\star}}+L \ell_{y}^{\star} \Theta_{y}^{(4)}=-\frac{2}{5 !} \frac{\partial^{5} \partial U_{x}^{(0)}}{\partial y^{\star 5}},
$$

or, in dimensional variables and denoting explicitly the observable corrections $\widetilde{U}_{x}^{(4)}=\varepsilon^{4} U_{x}^{(4)}, \widetilde{\Theta}_{x}^{(4)}=\varepsilon^{4} \Theta_{x}^{(4)}$ :

$$
\frac{\partial \widetilde{U}_{x}^{(4)}}{\partial y}+\ell_{y} \widetilde{\Theta}_{y}^{(4)}=-\ell^{4} \frac{2}{5 !} \frac{\partial^{5} \partial U_{x}^{(0)}}{\partial y^{5}} .
$$

Let us now replace (31)-(32) in the balance equation (30). This leads to

$$
\begin{aligned}
R_{x} \varepsilon^{2} \ell_{y}^{\star 4}\left(\frac{2}{4 !} \frac{\partial^{4} U_{x}^{(0)}}{\partial y^{\star 4}}\right. & \left.+O\left(\varepsilon^{4}\right)\right) \\
= & \ell_{x}^{\star 2}\left(\frac{\partial^{2} U_{x}^{(0)}}{\partial x^{\star 2}}+\varepsilon^{2} \ell_{x}^{\star 2}\left\{\frac{\partial^{2} U_{x}^{(2)}}{\partial x^{\star 2}}+\frac{2}{4 !} \frac{\partial^{4} U_{x}^{(0)}}{\partial x^{\star 4}}\right\}\right)+O\left(\varepsilon^{4}\right) .
\end{aligned}
$$

To exploit this equation, it is necessary to weigh the effects of bending (left-hand side term) and extension (right-hand side term). These effects are a consequence of both the mechanical properties of considered system and of the nature of the admitted kinematics. The mechanical parameters which we choose will introduce the high contrast condition (8), which is expressed by

$$
R_{x}=R_{x}^{\star} \varepsilon^{2}
$$


Regarding the nature of the macroscopic kinematics, we are led thus to distinguish between low or high contrast situations in the axial and the transverse gradient.

5.1. Low contrast between axial and transverse gradient of $\boldsymbol{U}_{\boldsymbol{x}}$. We consider here macroscopic kinematics where axial and transverse components of the displacement gradient $U_{x}$ are of the same order, i.e.,

$$
\frac{\partial U_{x}^{(0)}}{\partial x^{\star}}=O\left(\frac{\partial U_{x}^{(0)}}{\partial y^{\star}}\right) .
$$

This estimate explicitly means that the axial and transverse variations have as a common evolution characteristic value $O(L)$. This hypothesis is usually considered in the case of an elastic composite medium where the terms of the strain tensor components are assumed to be of the same order. In this case, we obtain successively (each relative to the orders $\varepsilon^{0}, \varepsilon^{2}, \varepsilon^{4}$ )

$$
\begin{aligned}
\frac{\partial^{2} U_{x}^{(0)}}{\partial x^{\star 2}} & =0, \\
\frac{\partial^{2} U_{x}^{(2)}}{\partial x^{\star 2}}+\frac{2}{4 !} \frac{\partial^{4} U_{x}^{(0)}}{\partial x^{\star 4}} & =0, \\
\frac{\partial^{2} U_{x}^{(4)}}{\partial x^{\star 2}}+\frac{2}{4 !} \frac{\partial^{4} U_{x}^{(2)}}{\partial x^{\star 4}}+\frac{2}{6 !} \frac{\partial^{6} U_{x}^{(0)}}{\partial x^{\star 6}} & =\left(\ell_{x}^{\star}\right)^{-2} R_{x}^{\star} \ell_{y}^{\star 4} \frac{2}{4 !} \frac{\partial^{4} U_{x}^{(0)}}{\partial y^{\star 4}} .
\end{aligned}
$$

By simplifying and returning to the dimensional variables and observable correctors, we deduce that

$$
\begin{aligned}
& \frac{E_{x} A_{x}}{\ell_{y}} \frac{\partial^{2} U_{x}^{(0)}}{\partial x^{2}}=0, \\
& \frac{E_{x} A_{x}}{\ell_{y}} \frac{\partial^{2} \widetilde{U}_{x}^{(2)}}{\partial x^{2}}=0, \\
& \frac{E_{x} A_{x}}{\ell_{y}} \frac{\partial^{2} \widetilde{U}_{x}^{(4)}}{\partial x^{2}}=\frac{E_{y} I_{y}}{\ell_{x}} \frac{\partial^{4} U_{x}^{(0)}}{\partial y^{4}} .
\end{aligned}
$$

Equations (37)-(38) mean that the tension of the fibers oriented along $x$ is constant to the accuracy $\varepsilon^{4}$. Only by considering the order 4 of the correctors, the tension of the beams varies due to the bending of orthogonal beams, as indicated by (39).

The assumption (36) obviously can not cover all cases of loading. In particular, it is not predictive if the lattice is subjected to a uniaxial extension in a direction that does not coincide with one of the directions of the fibers' arrays (see Figure 2). Indeed, if one considers the "red" fiber in Figure 2 (which presents experimental evidence), it is clear that its state of tension cannot be constant; its tension is not vanishing in the clamping but it clearly vanishes at the free end. To describe these 


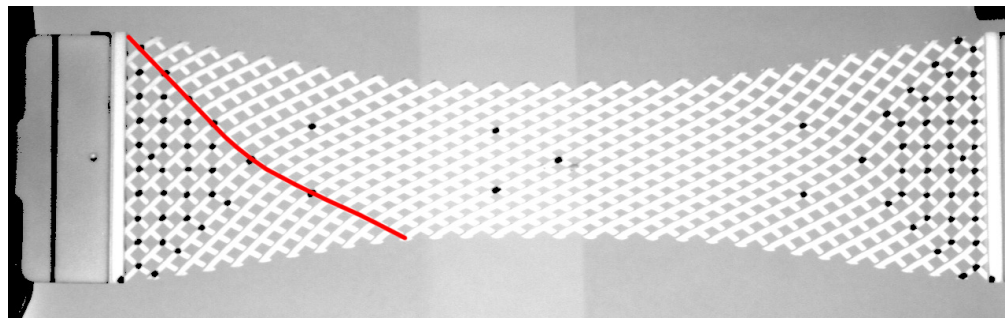

Figure 2. An example of standard bias extension test, courtesy of Tomasz Lekszycki, Marek Pawlikowski and Roman Grygoruk.

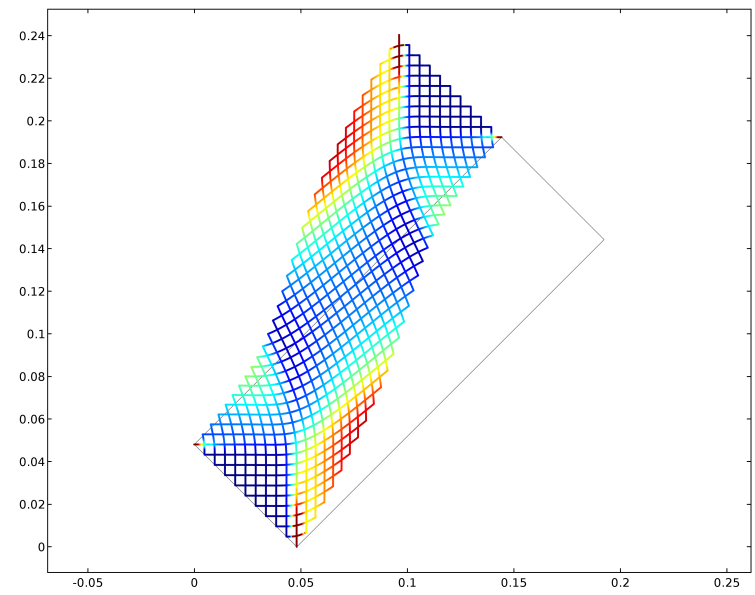

Figure 3. An example of generalized bias test while imposing a shear displacement.

situations it is necessary to change the too restrictive hypothesis (36) by allowing a strong contrast between the axial and transverse components of the gradient of the macroscopic displacement.

5.2. Strong contrast between axial and transverse gradient of $\boldsymbol{U}_{\boldsymbol{x}}$. Thus we are lead to consider macroscopic kinematics where the transverse gradient $\partial U_{x}^{(0)} / \partial y^{\star}$ dominates in comparison to the axial gradient $\partial U_{x}^{(0)} / \partial x^{\star}$, i.e.,

$$
\frac{\partial U_{x}^{(0)}}{\partial x^{\star}} \ll \frac{\partial U_{x}^{(0)}}{\partial y^{\star}} .
$$

This assumption reflects the fact that the axial characteristic length of variation of $U_{x}$ (denoted $L_{x, a}$ ) is much larger than the transverse (denoted $L_{x, t}$ ) length (see, e.g., experimental evidence presented in [dell'Isola et al. 2016d]). This is a consequence of the high shear deformability of the pantographic network compared to its deformability in the axis of the fibers. 
To assure scale separation, we are thus lead to consider as a reference length the smaller between the two, i.e., $L=L_{x, t}$, so that

$$
\frac{\partial^{i} U_{x}^{(0)}}{\partial x^{\star i}}=\frac{O\left(U_{x}^{(0)}\right)}{\left(L_{x, a} / L\right)^{i}}=O\left(U_{x}^{(0)}\right)\left(\frac{L_{x, t}}{L_{x, a}}\right)^{i}, \quad \frac{\partial^{j} U_{x}^{(0)}}{\partial y^{\star j}}=\frac{O\left(U_{x}^{(0)}\right)}{\left(L_{x, t} / L\right)^{j}}=O\left(U_{x}^{(0)}\right) .
$$

Once we consider $L_{x, t}=\varepsilon^{2} L_{x, a}$, we have

$$
\begin{aligned}
\frac{\partial U_{x}^{(0)}}{\partial x^{\star}} & =\varepsilon^{2} O\left(\frac{\partial U_{x}^{(0)}}{\partial y^{\star}}\right), \\
\frac{\partial^{2} U_{x}^{(0)}}{\partial x^{\star 2}} & =\varepsilon^{4} O\left(\frac{\partial^{4} U_{x}^{(0)}}{\partial y^{\star 4}}\right) .
\end{aligned}
$$

The presence of a contrast in the components of the strain tensor as given by (41) is unconventional in elastic composite mediums but arises naturally in the case of weakly compressible viscous fluid (where the trace of the strain rate tensor is negligible compared to its deviatoric component) or in beams and plates (where deformations in the section of the beam - or in the thickness of the plate - are negligible). This contrast is present also in pantographic sheets.

Replacing the estimate (42) into (34) we get at the leading order

$$
\frac{\partial^{2} U_{x}^{(0)}}{\partial x^{\star 2}}=\left(\ell_{x}^{\star}\right)^{-2} R_{x}^{\star} \ell_{y}^{\star 4} \frac{2}{4 !} \frac{\partial^{4} U_{x}^{(0)}}{\partial y^{\star 4}},
$$

or, returning to the dimensional variables and normalizing by introducing the surface of the periodic cell, we have

$$
\frac{E_{x} A_{x}}{\ell_{y}} \frac{\partial^{2} U_{x}^{(0)}}{\partial x^{2}}=\frac{E_{y} I_{y}}{\ell_{x}} \frac{\partial^{4} U_{x}^{(0)}}{\partial y^{4}} .
$$

This equation indicates that the normal force (left-hand side) varies at the first order in the beams due to the shear force exerted by the orthogonal beams (right-hand side). This is made possible because the transverse gradient is of two orders of magnitude higher than that of the extension gradient (see again (41)).

Moreover, we note that (43) is more general than (37) and it is needed in the considered mechanical system. Moreover, (43) degenerates to the (37) when $\partial U_{x}^{(0)} / \partial x \gg \varepsilon^{2} \partial U_{x}^{(0)} / \partial y$. We will use in what follows the description (43), which applies to more general kinematics.

5.3. Synthesis of obtained results. The above results, derived from the system (13)-(14) for the variables $U_{x}^{(0)}$ and $\Theta_{y}^{(0)}$ is transposed by a similar analysis of the system (15)-(16) to the variables $U_{y}^{(0)}$ and $\Theta_{x}^{(0)}$. The description in small deformations of the orthogonal pantographic lattice is therefore obtained by restricting the analysis to the dominant order (for simplicity, the exponents of order ${ }^{(0)}$ are 
removed):

$$
\begin{aligned}
& \frac{E_{x} A_{x}}{\ell_{y}} \frac{\partial^{2} U_{x}}{\partial x^{2}}=\frac{E_{y} I_{y}}{\ell_{x}} \frac{\partial^{4} U_{x}}{\partial y^{4}}, \\
& \frac{E_{y} A_{y}}{\ell_{x}} \frac{\partial^{2} U_{y}}{\partial y^{2}}=\frac{E_{x} I_{x}}{\ell_{y}} \frac{\partial^{4} U_{y}}{\partial x^{4}},
\end{aligned}
$$

with the addition of the relationships between the transverse gradients and rotations:

$$
\frac{\partial U_{x}}{\partial y}+\sqrt{\frac{\ell_{y}}{\ell_{x}}} \Theta_{y}=0, \quad \frac{\partial U_{y}}{\partial x}-\sqrt{\frac{\ell_{x}}{\ell_{y}}} \Theta_{x}=0 .
$$

Remark that (44)-(45) can be rewritten by introducing two intrinsic characteristic lengths $\eta_{x}$ and $\eta_{y}$ as follows:

$$
\frac{\partial^{2} U_{x}}{\partial x^{2}}=\eta_{x}^{2} \frac{\partial^{4} U_{x}}{\partial y^{4}}, \quad \frac{\partial^{2} U_{y}}{\partial y^{2}}=\eta_{y}^{2} \frac{\partial^{4} U_{y}}{\partial x^{4}},
$$

where

$$
\eta_{x}^{2}:=\frac{\ell_{y} E_{y} I_{y}}{\ell_{x} E_{x} A_{x}}, \quad \eta_{y}^{2}:=\frac{\ell_{x} E_{x} I_{x}}{\ell_{y} E_{y} A_{y}} .
$$

It is clear that the physics of the system is governed by these internal intrinsic lengths which differ from the size of the cell.

The model governed by (44)-(45) is of the type of a conservative generalized continuum medium having deformation energy that depends on first- and secondorder gradients of displacement (see [Alibert et al. 2003; Seppecher et al. 2011]). Its evolution is ruled by two displacement fields which are independent and uncoupled, i.e., $U_{x}$ and $U_{y}$. This particular behavior is within the framework of the second gradient continua where the internal actions are described by a stress symmetric tensor $\boldsymbol{T}$ of order two and by a hyperstress tensor $\mathscr{T}$ of third order. To make the identification, we pose (44)-(45) in the following form, where the differential operator DIV denotes the Lagrangian divergency:

$$
\operatorname{DIV}(\boldsymbol{T}-\operatorname{DIV}(\mathscr{T}))=0
$$

and where we used the definitions

$$
\boldsymbol{T}:=\frac{1}{2} C \cdot\left(\nabla \boldsymbol{U}+\nabla \boldsymbol{U}^{\mathrm{t}}\right), \quad \mathscr{T}:=D \cdot \nabla \nabla \boldsymbol{U}
$$

in which the symbol "." denotes the repeated index saturation between different order tensors and superscript " $\mathrm{t}$ " the transposition of second-order tensors. The elasticity tensors $C$ and $D$ of order four and six respectively have the particular 
form defined by their components as

$$
\begin{aligned}
C_{a b c d} & :=\frac{E_{x} A_{x}}{\ell_{y}} \delta_{a x} \delta_{b x} \delta_{c x} \delta_{d x}+\frac{E_{y} A_{y}}{\ell_{x}} \delta_{a y} \delta_{b y} \delta_{c y} \delta_{d y}, \\
D_{a b c d e f} & :=\frac{E_{y} I_{y}}{\ell_{x}} \delta_{a x} \delta_{b y} \delta_{c y} \delta_{d x} \delta_{e y} \delta_{f y}+\frac{E_{x} I_{x}}{\ell_{y}} \delta_{a y} \delta_{b x} \delta_{c x} \delta_{d y} \delta_{e x} \delta_{f x} .
\end{aligned}
$$

Remark that (separately) the tensors $C$ and $D$ are not coercive. For instance, pure shear deformations do not have any first gradient energetic content, while any affine displacement field does not have any second gradient energetic content. This is consistent with the existence, for pantographic sheets, of floppy modes as nullifiers of deformations energy, in addition to the standard rigid motions. Nevertheless, considering both first and second gradient energies together, the system can be qualified as "subcoercive" when suitable boundary conditions are imposed in such a way that floppy modes are excluded. In the framework of this requirement, the set of admissible displacements is restricted if compared to the one needed in first gradient theory, where only rigid body motions are excluded. We conjecture that in the set of considered admissible displacements, the total deformation energy is definite positive and leads to well-posed problems. This seems physically wellgrounded and is confirmed by all the performed numerical simulations presented in the last section.

To identify the class of physically meaningful boundary conditions, in the next section we more closely study the structure of the considered energy.

5.4. Energy formulation and boundary conditions for pantographic lattices. The general framework of second gradient continua (as formulated in [dell'Isola et al. $2015 \mathrm{c}]$ ) enables, by means of the energy formulation and of the divergence theorem, to specify the boundary conditions which can be consistently considered as applicable to them.

Let $\mathscr{B}$ be a bidimensional medium whose elastic energy depends upon the displacement gradient $\nabla \boldsymbol{U}$ acting on the second-order stress tensor $\boldsymbol{T}$ and upon the second displacement gradient acting on the third-order hyperstress tensor $\mathscr{T}$. In this case the energy $W$ of $\mathscr{B}$ is

$$
2 W=\int_{\mathscr{B}}(\boldsymbol{T}: \nabla \boldsymbol{U}+\mathscr{T}: \nabla \nabla \boldsymbol{U})
$$

Transforming this expression through successive integrations by parts enables us to make the link between the variation of internal energy and the energy supplied at the boundary of $\mathscr{B}$ (see, e.g., [dell'Isola et al. 2015c]). As the considered system is bidimensional, the boundary consists of a set of regular edges $\partial B$ with unit normal $\boldsymbol{n}$ and wedges $\partial \partial \mathscr{B}$ consisting of the union of a finite number $N$ of vertices $S_{I}$, i.e., 
$\partial \partial \mathscr{B}=\cup S_{I}=\{S\}$. For our calculations we introduce Levi-Civita tensorial notation, keeping track of the covariance and contravariance nature of the considered tensorial quantities and we use Latin indices for Eulerian components and Greek letters for Lagrangian components. Therefore, we have

$$
\begin{aligned}
\delta W & =\int_{\mathscr{R}}(\boldsymbol{T}: \nabla \delta \boldsymbol{U}+\mathscr{T} \vdots \nabla \nabla \delta \boldsymbol{U})=\int_{\mathscr{乃}}\left(T_{i}^{\beta} \delta U_{, \beta}^{i}+\mathscr{T}_{i}^{\alpha \beta} \delta U_{, \alpha \beta}^{i}\right) \\
& =\int_{\mathscr{乃}}\left(-T_{i, \alpha}^{\alpha} \delta U^{i}-\mathscr{T}_{i, \beta}^{\alpha \beta} \delta U_{, \alpha}^{i}\right)+\int_{\mathscr{乃}}\left(T_{i}^{\beta} \delta U^{i}+\mathscr{T}_{i}^{\alpha \beta} \delta U_{, \alpha}^{i}\right)_{, \beta} \\
& =\int_{\mathscr{乃}}\left(-T_{i, \alpha}^{\alpha} \delta U^{i}-\mathscr{T}_{i, \beta}^{\alpha \beta} \delta U_{, \alpha}^{i}\right)+\int_{\partial \mathscr{P}}\left(T_{i}^{\beta} \delta U^{i}+\mathscr{T}_{i}^{\alpha \beta} \delta U_{, \alpha}^{i}\right) n_{\beta} \\
& =\int_{\mathscr{R}}\left(\mathscr{T}_{i, \beta \alpha}^{\alpha \beta}-T_{i, \alpha}^{\alpha}\right) \delta U^{i}+\int_{\partial \mathscr{P}}\left(\left(T_{i}^{\beta}-\mathscr{T}_{i, \alpha}^{\beta \alpha}\right) \delta U^{i}+\mathscr{T}_{i}^{\alpha \beta} \delta U_{, \alpha}^{i}\right) n_{\beta} .
\end{aligned}
$$

Using the equilibrium equation (47), the first integral in the right-hand side vanishes. Furthermore, the last term of the second integral can be decomposed into a tangent and a normal contribution. As the considered system is bidimensional, the boundary $\partial B$ includes a set of regular edges. Thus, introducing the projector on the tangent direction of $\partial B, P=I-n \otimes n$ (hence $P_{\alpha}^{\gamma}=\delta_{\alpha}^{\gamma}-n_{\alpha} n^{\gamma}$ and $P . P=P$ ), we have

$$
\begin{aligned}
& \delta W=\int_{\partial \mathscr{\beta}}\left(\left(T_{i}^{\beta}-\mathscr{T}_{i, \alpha}^{\beta \alpha}\right) n_{\beta} \delta U^{i}+\left(\mathscr{T}_{i}^{\alpha \beta} n_{\alpha} n_{\beta}\right) \delta U_{, \gamma}^{i} n^{\gamma}+\mathscr{T}_{i}^{\alpha \beta} n_{\beta} P_{\alpha}^{\delta} \delta U_{, \gamma}^{i} P_{\delta}^{\gamma}\right) \\
& =\int_{\partial \mathscr{P}}\left(\left(T_{i}^{\beta}-\mathscr{T}_{i, \alpha}^{\beta \alpha}\right) n_{\beta} \delta U^{i}+\left(\mathscr{T}_{i}^{\alpha \beta} n_{\alpha} n_{\beta}\right) \delta U_{, \gamma}^{i} n^{\gamma}\right. \\
& \left.+\left(\mathscr{T}_{i}^{\alpha \beta} n_{b} P_{\alpha}^{\delta} \delta U^{i}\right)_{, \gamma} P_{\delta}^{\gamma}-\left(\mathscr{T}_{i}^{\alpha \beta} n_{\beta} P_{\alpha}^{\delta}\right)_{, \gamma} P_{\delta}^{\gamma} \delta U^{i}\right) \\
& =\int_{\partial \mathscr{\Re}}\left(\left(T_{i}^{\beta}-\mathscr{T}_{i, \alpha}^{\beta \alpha}\right) n_{\beta}-\left(\mathscr{T}_{i}^{\alpha \beta} n_{\beta} P_{\alpha}^{\delta}\right)_{, \gamma} P_{\delta}^{\gamma}\right) \delta U^{i}+\int_{\partial \mathscr{\Re}}\left(\mathscr{T}_{i}^{\alpha \beta} n_{\alpha} n_{\beta}\right) \delta U_{, \gamma}^{i} n^{\gamma} \\
& +\int_{\partial \partial \mathscr{P}} \mathscr{T}_{i}^{\alpha \beta} n_{\beta} P_{\alpha}^{d} N_{\delta} \delta U^{i}
\end{aligned}
$$

On the last integral on $\partial \partial B=\{S\}$, the vectors $\boldsymbol{n}$ and $\boldsymbol{N}$ take the values $\boldsymbol{n}^{ \pm}$and $\boldsymbol{N}^{ \pm}$ defined on the both sides of the discontinuity of the edges. This is also the case for $\mathcal{T}$ and $P$. On each side, the vector $N$ is the tangent vector to $\partial B$ which is the outer pointing normal to the border of $\partial B$; see Figure 4. Thus $P^{+} . N^{+}=N^{+}$and $P^{-} . N^{-}=N^{-}$. In a condensed way, we can write (where the symbol $\|$refers to the tangent projection of a tensor on $\partial B$ )

$$
\begin{aligned}
& \int_{\mathscr{乃}}(\boldsymbol{T}: \nabla \delta \boldsymbol{U}+\mathscr{T} \vdots \nabla \nabla \delta \boldsymbol{U}) \\
& =\int_{\partial \mathscr{\Re}}\left((T-\mathrm{DIV} \mathscr{T}) \cdot n-\mathrm{DIV}_{\|} \mathscr{T}_{\|}\right) \cdot \delta U+(\mathscr{T} \cdot n \cdot n) \delta \frac{\mathrm{d} U}{\mathrm{~d} n}+\sum_{\{S\}}[\mathcal{T} \cdot n \cdot N] . \delta U
\end{aligned}
$$




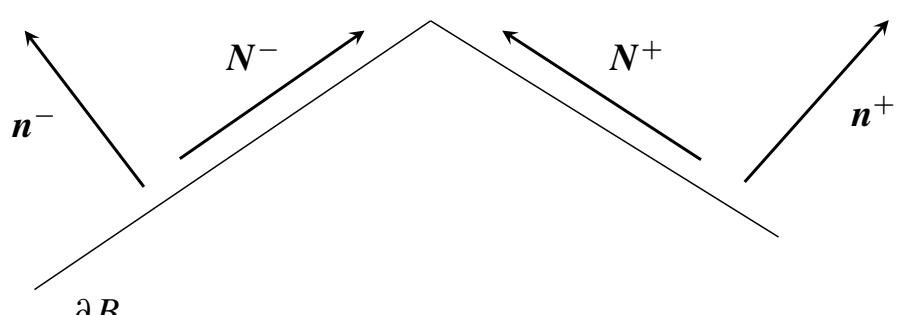

Figure 4. Sketch of vectors $\boldsymbol{n}$ and $\boldsymbol{N}$ on the boundaries.

with

$$
[\mathcal{T} \cdot n \cdot N]=\left(\mathscr{T}^{+} \cdot n^{+} \cdot N^{+}\right)+\left(\mathscr{T}^{-} \cdot n^{-} \cdot N^{-}\right) .
$$

The previous integration by parts implies that in order to have well-posed problems, one has to suitably assign boundary conditions in a specific way (see, e.g., [Mindlin 1964]). In particular, on any regular point belonging to the set of edges วभ one can assign:

- either a component $U^{i}$ or its dual quantity $\left(T_{i}^{b}-\mathscr{T}_{i, \alpha}^{\beta \alpha}\right) n_{\beta}-\left(\mathscr{T}_{i}^{\alpha \beta} n_{\beta} P_{\alpha}^{\delta}\right)_{, \gamma} P_{\delta}^{\gamma}$. In the case of the rectilinear edge $\partial \mathscr{B}$ the normal and the projector are constants and this expression simplifies into $\left(T_{i}^{\beta}-\mathscr{T}_{i, \alpha}^{\beta \alpha}-\mathscr{T}_{i, \gamma}^{\alpha \beta}\left(\delta_{\alpha}^{\gamma}-n_{\gamma} n_{\alpha}\right)\right) n_{\beta}$;

- either a component of displacement normal gradient, $\mathrm{d} U^{i} / \mathrm{d} n$, or its dual quantity $\left(\mathscr{T}_{i}^{\alpha \beta} n_{a} n_{\beta}\right)$.

Moreover, on vertices constituting $\partial \partial B=\{S\}$ one can assign:

- either a component $U^{i}$ or its dual quantity $\left[\mathscr{T}_{i}^{\alpha \beta} n_{b} N_{\alpha}\right]$ which expresses the discontinuity of the wedge on the vertices (see Figure 4).

Therefore the weak condition for equilibrium is given by

$$
\delta W=\int_{\partial \mathscr{B}} f_{i}^{\mathrm{ext}} \delta U^{i}+\tau_{i}^{\mathrm{ext}} \delta U_{, \gamma}^{i} n^{\gamma}+\int_{\partial \partial \mathscr{B}} F_{i}^{\mathrm{ext}} \delta U^{i},
$$

where external actions are given by forces per unit line $f_{i}^{\text {ext }}$ but also by concentrated forces on vertices $F_{i}^{\text {ext }}$ and by double forces per unit line $\tau_{i}^{\text {ext }}$ (see [Germain 1973]).

One can apply these results to the particular pantographic lattice considered, where the preferred orthogonal directions $x$ and $y$ coincide with the array of material fibers. In the context of small deformations, the Lagrangian and Eulerian coordinate systems can be identified. In the pantographic case given by (49) and (50) the deformation energy is given by

$2 W=\int_{\mathscr{R}}\left(\frac{E_{x} A_{x}}{\ell_{y}}\left(U_{, x}^{x}\right)^{2}+\frac{E_{y} A_{y}}{\ell_{x}}\left(U_{, y}^{y}\right)^{2}+\frac{E_{y} I_{y}}{\ell_{x}}\left(U_{, y y}^{x}\right)^{2}+\frac{E_{x} I_{x}}{\ell_{y}}\left(U_{, x x}^{y}\right)^{2}\right) \mathrm{d} x \mathrm{~d} y$ 
so we have that, as $\boldsymbol{T}=\partial W / \partial \nabla U$ and $\mathscr{T}=\partial W / \partial \nabla \nabla U$, the only nonvanishing components of stress and hyperstress are

$$
\begin{aligned}
& T_{x}^{x}=\frac{E_{x} A_{x}}{\ell_{y}} U_{, x}^{x}, \quad T_{y}^{y}=\frac{E_{y} A_{y}}{\ell_{x}} U_{, y}^{y}, \\
& \mathscr{T}_{y}^{x x}=\frac{E_{x} I_{x}}{\ell_{y}} U_{, x x}^{y}, \quad \mathscr{T}_{x}^{y y}=\frac{E_{y} I_{y}}{\ell_{x}} U_{, y y}^{x} .
\end{aligned}
$$

Thus, for the pantographic sheet, the two types of kinematic and static (more often called natural) boundary conditions which apply to a straight line edge of normal $\boldsymbol{n}=n_{x} \boldsymbol{e}_{x}+n_{y} \boldsymbol{e}_{y}$ take the following form:

$$
\begin{aligned}
& U^{x} \text { dual of } T_{x}^{x} n_{x}+\left(-\mathscr{T}_{x, y}^{y y}\left(1+n_{x}^{2}\right)+\mathscr{T}_{x, x}^{y y} n_{x} n_{y}\right) n_{y}, \quad \frac{\mathrm{d} U^{x}}{\mathrm{~d} n} \text { dual of } \mathscr{T}_{x}^{y y} n_{y}^{2}, \\
& U^{y} \text { dual of } T_{y}^{y} n_{y}+\left(-\mathscr{T}_{y, x}^{x x}\left(1+n_{y}^{2}\right)+\mathscr{T}_{y, y}^{x x} n_{x} n_{y}\right) n_{x}, \quad \frac{\mathrm{d} U^{y}}{\mathrm{~d} n} \text { dual of } \mathscr{T}_{y}^{x x} n_{x}^{2} .
\end{aligned}
$$

On the vertices, noting that $\boldsymbol{n}$ and $\boldsymbol{N}$ are orthogonal, the flux dual to the displacement components $U^{x}$ and $U^{y}$ are respectively $\mathscr{T}_{x}^{-y y} n_{x}^{-} n_{y}^{-}-\mathscr{T}_{x}^{+y y} n_{x}^{+} n_{y}^{+}$and $\mathscr{T}_{y}^{-x x} n_{x}^{-} n_{y}^{-}-\mathscr{T}_{y}^{+x x} n_{x}^{+} n_{y}^{+}$. Remark that they vanish when the vertex angle is $\frac{1}{2} \pi$ and the boundary is parallel to the fibers.

For a better physical insight, consider for instance a straight edge oriented along the direction $x$, then $\boldsymbol{n}=\boldsymbol{e}_{y}$ and the boundary condition simplifies to

$$
\begin{array}{cc}
U^{x} \text { dual of }-\mathscr{T}_{x, y}^{y y}=-\frac{E_{y} I_{y}}{\ell_{x}} U_{, y y y}^{x}, & \frac{\mathrm{d} U^{x}}{\mathrm{~d} y} \text { dual of } \mathscr{T}_{x}^{y y}=\frac{E_{y} I_{y}}{\ell_{x}} U_{, y y}^{x}, \\
U^{y} \text { dual of } T_{y}^{y}=\frac{E_{y} A_{y}}{\ell_{x}} U_{, y}^{y}, \quad \frac{\mathrm{d} U^{y}}{\mathrm{~d} y} \text { dual of } \mathscr{T}_{y}^{x x} n_{x}^{2}=0 .
\end{array}
$$

These boundary conditions on the lattice can be easily understood by recalling standard beam theory. They show this:

- A virtual displacement tangent to the fiber materializing the edge develops energy due to the shear forces in the orthogonal fibers.

- A virtual displacement normal to the edge fiber develops energy due to the tension forces in the orthogonal fibers.

- A rotation of the edge fiber develops energy due to the couple in the orthogonal fibers.

- No energy is developed (in small deformations) by the extension of the orthogonal fibers associated with the couple of the edge fiber.

If the vertex at the end of the edge along $x$ presents an internal angle $\alpha$, the static quantities dual to the components $U^{x}$ and $U^{y}$ are respectively the weighted couples $\mathscr{T}_{x}^{+y y} \frac{1}{2} \sin (2 \alpha)$ and $-\mathscr{T}_{y}^{+x x} \frac{1}{2} \sin (2 \alpha)$. No energy is developed if the vertex angle is $\frac{1}{2} \pi$ with edges oriented along the fibers. 
A similar interpretation applies when the straight edge presents an angle with the fiber orientation; however, due to the coupling between the forces and couples in different directions, the physical interpretation of the boundary condition becomes more difficult.

In this paper, for the sake of simplicity, we consider only imposed boundary conditions on displacements and displacement gradients and we consider weak form (55) of equilibrium conditions, so that no dual boundary conditions (on forces or double forces) are assigned. In further papers, we will consider more general situations.

\section{Some equilibrium shapes of linear pantographic sheets: numerical simulations}

Pantographic sheets have an exotic behavior which is not only characterized by their anisotropy as evidenced by:

(1) their vanishing resistance to shear deformation, and

(2) their significant resistance to elongation along fibers, and also by their capacity to resist variations in their so-called "geodesic curvature" (see [dell'Isola and Steigmann 2015; Steigmann and dell'Isola 2015; Giorgio et al. 2015; Giorgio et al. 2016]), i.e., the changes of curvature of material curves induced by inplane displacements.

Moreover, in their deformation patterns one can observe the onset of inner boundary layers where bending of constituting beams is concentrated, as suggested by the existence of the intrinsic characteristic lengths $\eta_{x}$ and $\eta_{y}$.

The aim of this section is to provide numerical illustrations of the theoretical developments presented in the previous sections. The considered examples may seem purely academic or dictated simply by the taste of investigating mathematical structures (see [Dieudonné 1987]). Although we indeed consider that scientific knowledge is based on the study of exemplary cases; see [Russo 2004; Hero/Woodcroft 1851; Heath 1921a; 1921b; Archimedes/Heath 1897; 1912]. In addition, a potential application of the presented results concern the forming of fiber reinforced composites (see, e.g., [Cao et al. 2008; Launay et al. 2008; d'Agostino et al. 2015; Harrison 2016; Abdiwi et al. 2013; Nikopour and Selvadurai 2014]).

All the presented numerical simulations are obtained by a code created using COMSOL Multiphysics. The homogenized energy introduced in this paper (56) is minimized by using the package "Weak Form PDE" and by introducing standard third-order Hermite finite elements. While the used code is surely not optimized for the introduced problem (we believe that the recently developed numerical methods would be more efficient, see, e.g., [Cazzani et al. 2016a; 2016b; Greco and Cuomo 2013; 2014; 2015; 2016; Turco and Aristodemo 1998; Beirão da Veiga 
et al. 2008; Della Corte et al. 2016]), its rate of convergence seems satisfactory for getting preliminary results concerning the behavior of the simplest structures; actually, it is based on the introduction of an auxiliary tensor field which appears in the deformation energy and is equated to the displacement gradient by means of suitable fields of Lagrange multipliers. Remark also that all presented numerical simulations are really and intrinsically mesh-independent, because of the properties of the introduced continuum model, where the second gradient of displacement is at the same time modeling the relevant physical properties and supplies a regularizing effect on equilibrium equations.

In the presented simulations we have chosen a lattice made of square cells, so that $\ell_{x}=\ell_{y}=\ell$ and we have imposed that the $x$ and $y$ fibers have identical rectangular sections (having sides $a$ and $b$ ) and elastic moduli so that $E_{y}=E_{x}$, $I_{x}=I_{y}=\frac{1}{12} b a^{3}$ and $A_{x}=A_{y}=a b$. As a consequence we have that

$$
\eta^{2}=\eta_{x}^{2}=\eta_{y}^{2}=\frac{I_{x}}{A_{x}}=\frac{I_{y}}{A_{y}}=\frac{1}{12} a^{2} .
$$

The values $a=0.9 \mathrm{~mm}$ and $b=1.6 \mathrm{~mm}$ are used for pantographic structures, following experimental measurements (see [dell'Isola et al. 2015b]) having rectangular sections. Young's modulus is $1600 \mathrm{MPa}$. We remark that the elastica model for beams is applicable in the considered situation as $\ell=4.95 \mathrm{~mm}$ and the number of cells is sufficiently large $(L=42.42 \times \ell)$ to apply the homogenized model.

In the following subsections, we present the numerical simulation of bias tests in different configurations. First, we consider rectangular specimens undergoing standard bias test in extension, but also in shear and bending. The results demonstrate the ability of the model to catch the occurrence of highly nonhomogeneous deformation patterns with inner boundary layer, and illustrate the dependance of the pattern on the different imposed deformations. Second, the same type of bias tests are performed on circular specimens in order to investigate the role of the sample geometry on the equilibrium shape and on the elastic energy distribution. Finally, extension and bending bias tests on circular specimens with a central squared of different orientations are simulated. The comparison with the previous cases evidence the effect of different boundary conditions.

In all the following figures, the black lines indicate the local actual orientation of the material fibers (which are orthogonal in the reference configuration), and the deformed shape is displayed together with the map of the stored energy density. All the calculations are performed in the framework of small deformations; however, for a better insight, the deformation is magnified in the figures.

6.1. Bias tests on rectangular pantographic sheet. We start by considering a pantographic sheet having a rectangular initial shape with the long side three times longer than the short one. 

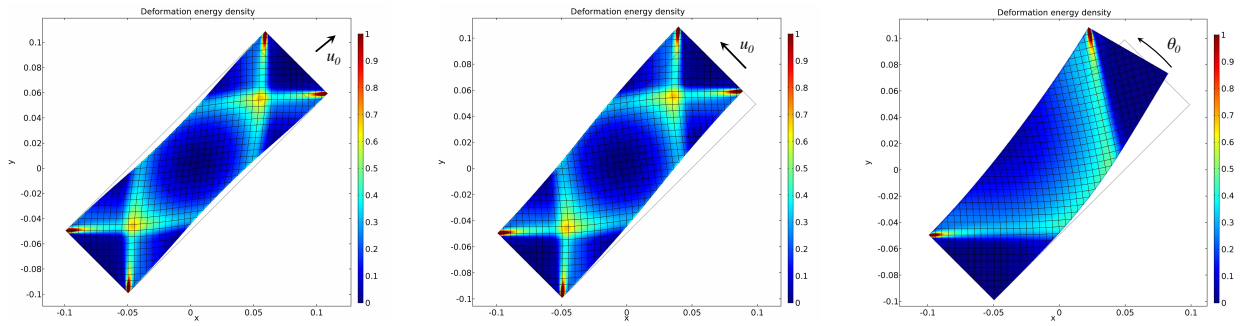

Figure 5. Equilibrium shapes of rectangular specimens submitted to extension (left) shear (center) and bending (right) displacement. The colors indicate the levels of stored energy density.

In the standard extension bias test the short sides are rigidly displaced in the direction of the long side. Figure 5, left, shows the small deformation pattern. Remark that the deformation energy is concentrated along the material lines constituted by some fibers and that, while the fibers are extensible in the present case, the structure of the deformed shape is similar to the one described by Pipkin [1980; $1981]$ in the case of inextensible fibers. Notice also that the distribution of deformation is strongly nonhomogeneous while respecting the symmetry of the loading. The simulation enables the identification of several zones with different kinematics. The partitioning of the specimen can be described as follows:

- The clamping zones that consists of two "isosceles triangles" with bases on the short sides. These areas do not sustain any significant deformation, neither in extension nor in shear, so the first gradient description (37) applies. The vanishing stored energy results here from the quasirigid body motion of these regions.

- The deformed zones outside of the aforementioned triangles, in which large shear deformations arise. In this highly sheared domain, the behavior is governed by the second gradient description (44)-(45). One distinguishes a central and four lateral subdomains of vanishing stored energy delimited by transition zones. The different subdomains correspond to the occurrence of local "floppy" modes. Indeed, because of the specific boundary condition, "floppy" modes on the whole specimen are forbidden. Nevertheless, the minimum of energy is attained by activating local floppy modes, far from the boundary conditions. This results in large parts of the body where the deformation energy is very close to vanishing.

- The transition zones between the different domains (of quasifloppy modes or quasimonolithic type) consist in the inner boundary layers where the bending of the fibers is concentrated to accommodate the different kinematics that prevails in the two regions in contact. Such layers, which concentrate the 

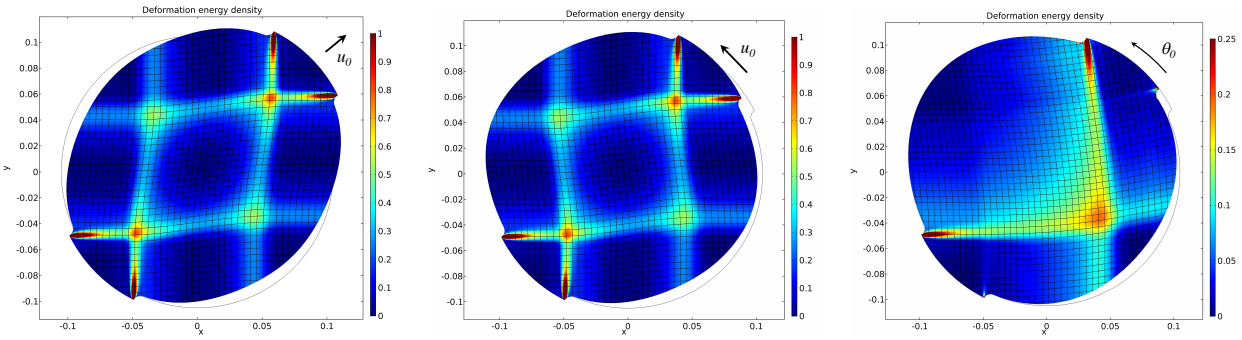

Figure 6. Equilibrium shapes of circular specimens submitted to extension (left) shear (center) and bending (right) displacement. The colors indicate the levels of stored energy density.

elastic energy, take place along material lines constituted by fibers and are characterized by large gradients of shear deformation. These specific features are the direct signature of the second gradient effects: a standard Cauchy continuum description (i.e., simple gradient description, as in (37)) would lead to a homogeneous deformation pattern and avoid the development of shear bands.

Furthermore, the qualitative and quantitative comparison of the numerical simulations plotted on Figure 5, left, and the experimental data presented in [dell'Isola et al. 2015b] clearly argue in favor of the second gradient description (44)-(45) for two reasons. First, the experimental deformation pattern is obviously nonhomogeneous with inner boundary layers whose structure presents a similar geometry as that observed in the simulation. Second, by making a best fit of the experimental data [dell'Isola et al. 2015b], the effective parameters of the second gradient continuum model have been identified. It happens that this "blind" procedure supplies exactly the same values of the effective parameters as the one calculated from the micro-macro upscaling procedure, once the geometric and mechanical properties of the cell beams of the sheet tested experimentally are taken.

In addition, simulations of shear bias tests with uniform lateral displacement imposed on the top side and bending bias tests with rotational displacement imposed on the top side (corresponding to a rigid body rotation centered in the middle of the specimen) have been done. The results are displayed in Figure 5, center and right, respectively. Similar general comments as done for the extension test still apply; however, the geometry of the shear bands and the energy distribution is modified. In particular, the partitioning of the specimen submitted to bending shows only one subdomain of quasifloppy mode which is confined between two quasimonolithic zones.

6.2. Bias test on circular pantographic sheets. Consider now the same bias test as the previous one, except that the rectangular pantographic sheet is replaced by a 
sheet of circular initial shape. The imposed deformations are obtained by clamping one circular arc and imposing a rigid displacement on the opposite one.

In Figure 6, left, we consider the extension imposed by a relative rigid translation of the two arcs in the direction of the common bisecting diameter. Note the great similarity of the deformation pattern obtained with rectangular and circular sheets, when focusing on the internal rectangular domain considered in the standard bias test. This means that, independently of rectangular or circular geometry of the sheet, the structure of the deformation pattern is kept (almost) unchanged when identical kinematic boundary conditions are imposed. Remark also that the largest lateral dimension of the circular sheet enables the inner boundary layers to extend and intersect. Thus the partitioning of the specimen is complemented by the appearance of two additional lateral subdomains.

These observations indicate that in the considered cases, the structure of the inner boundary layer (hence of the partitioning) mostly results from the geometry of the boundary conditions, while the extension of the shear bands depends on the geometry of the whole body. Furthermore, in the circular case, the activation of the deformation outside of the rectangle implies additional energy. Therefore, to reach the same displacement imposed at the boundaries, a larger force is required in the circular case than in the rectangular case.

In Figure 6, center, the relative rigid displacement is in the direction orthogonal to the bisecting diameter and imposes a shear to the specimen. The resulting shear deformation pattern is similar to the one activated in extension (Figure 6, left), but with different orientations of the inner boundary layers. The comparison with the case of rectangular sheet leads to similar comments as above.

In Figure 6, right, the relative displacement of the two arcs is obtained by fixing one arc and by rotating the second one with respect the center of the circle. Remark that the partitioning of the specimen is similar as in the rectangular case but here the boundary layers invade the whole body.

\subsection{Bias test on initially circular pantographic sheets with central holes. In the} same body considered in the previous subsection, a square hole (of $14 \times 14$ cells) is now carved in its central part. The initial orientation of the hole relative to the fibers varies from zero (i.e., the sides of the square are along the fibers) to $\frac{1}{8} \pi$ and $\frac{1}{4} \pi$ (i.e., the edges of the square are along and orthogonal to the extension displacement). These different cases enable the investigation of the effect of the hole on the deformation pattern and on the onset of inner boundary layers.

One may expect that when a hole is carved in a subdomain corresponding to quasifloppy modes, its influence should be negligible since in both cases the hole or floppy modes deformation occurs with no energy expense. However, if a hole intersects the deformation boundary layers in which the energy would be localized 

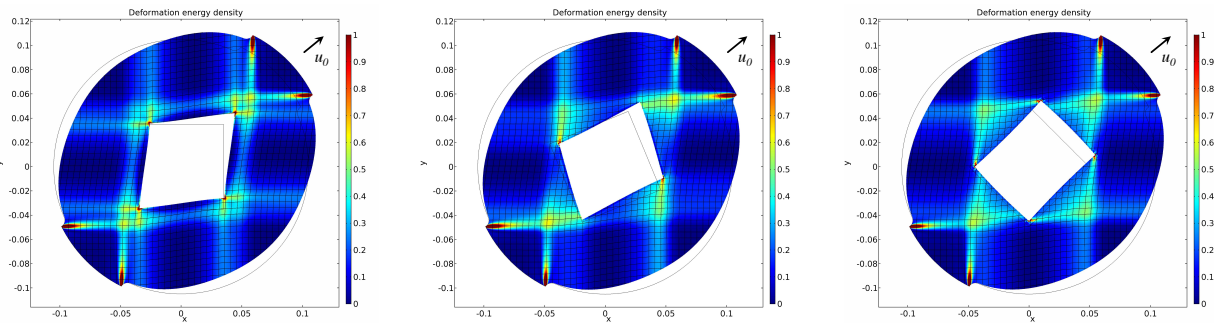

Figure 7. Equilibrium shapes for extension bias test of a circular sample with a squared hole: left, whose edges are oriented along fibers; center, rotated at an angle of $\frac{1}{8} \pi$; right, rotated at an angle of $\frac{1}{4} \pi$. The colors indicate the levels of stored energy density.
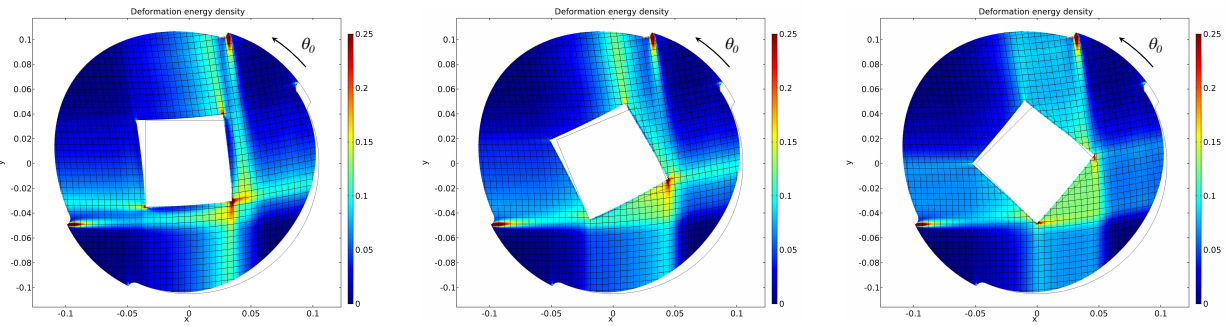

Figure 8. Equilibrium shapes for bending test of a circular sample with a squared hole: left, whose edges are oriented along fibers; center, rotated of an angle of $\pi / 8$; right, rotated of an angle of $\frac{1}{4} \pi$. The colors indicate the levels of stored energy density.

in absence of a hole, then the energy distribution is necessarily modified and so does the deformation patterns.

This is what is observed in the simulations. The edges of the carved hole are such that when oriented along the fibers, the hole almost belongs to the central floppy mode subdomain that arises in the noncarved sheet, while when rotated, two corners of the hole may cross the boundary layers of the intact sheet. In extension tests presented in Figures 7, left and right, the intersection of the carved hole with the deformation boundary layers of the intact body is relatively small and the deformation patterns are weakly disturbed. This is not the case in Figure 7, center, where the hole cuts the material lines, i.e., fibers in which the energy would be concentrated in an intact body. As a consequence, the deformation response and the partitioning of the specimen change significantly. The same trends are observed for the bending test: weak perturbations arise in Figure 8, left, but large and dramatic change appears in Figure 8, center and right, respectively. 


\section{Conclusions}

Pantographic sheets belong to the specific class of architectured materials whose mechanical behavior is characterized by:

- very high contrast of extensional and bending stiffness at microscopic level (see (35));

- very high contrasted gradients of displacement in the axial and transverse direction at the macrolevel (see (40));

- a microstructure which produces a discretely oriented, orthotropic material exhibiting an extreme anisotropy leading to the presence of two preferred material directions having very high extensional stiffness; this circumstance causes the onset of internal boundary layers where gradients of deformation may arise.

The standard (Cauchy or first gradient) continuum models were conceived under some implicit assumptions which do not allow for the description of all above listed extreme mechanical properties [dell'Isola et al. 2015a]. For this reason, it is needed to introduce, for pantographic sheets, a generalized continuum model by reconsidering, at the very beginning, the standard modeling procedure. Indeed a consistent model should allow for, in particular, the description of deformation gradient concentrations induced by imposed boundary displacements. To be driven in the construction of the most suitable model we use the asymptotic homogenization method extensively presented in [Boutin and Auriault 1993]. It allows for the rigorous construction of a description able to encompass all mentioned atypical properties.

Even if we limit ourselves to the case of linearized models in statics (and in the absence of body forces), we still get a nonstandard second gradient continuum model. Moreover, the advantage of used micro-macro upscaling procedure is that it allows us to determine firstly the atypical structure of the constitutive and balance equations and secondly all relative constitutive parameters (i.e., those specifying the first gradient and second gradient terms in deformation energy). This is done explicitly in terms of the microscopic properties of the elementary cells constituting the pantographic sheet. Furthermore, the presented micro-macro identification provides a design rule for pantographic bidimensional continua.

The main advances provided by this paper compared to some previous works on pantographic sheets lie in the three following points:

- The homogenization method leads to a clear and rigorous micro-macro identification procedure. Hence, the structure of the homogenized description and its parameters are explicitly deduced from the cell. This differs from the $a$ priori variational approaches where a macroscopic description is proposed 
but its applicability to specific microstructures can only be postulated. Besides, compared to direct numerical simulations at the microscale [dell'Isola et al. 2016b], the established model presents a great advantage, in terms of computational cost and also of in terms of physical understanding of the actual behavior.

- The description enables us to account for the low extensibility and the high bending deformability. It thus enables us to overcome the drawback of the studies that consider inextensible fibers [dell'Isola et al. 2016c].

- The physical insight of pantographic systems is improved by a simple interpretation of the macroscopic description: the tension in a fiber varies because of the shear forces transferred through the pivots by the orthogonal fibers. Even if the paper focuses on small deformations, this mechanism remains essentially the same (albeit complicated by the nonorthogonality of the fibers) at large deformation [dell'Isola et al. 2016d].

Note that we only consider small in-plane deformations. However, the same homogenization method can be extended to investigate buckling and out-plane deformation as discussed in [Giorgio et al. 2015; 2016].

The obtained models can be framed in the context of generalized continuum theories and immediately allows for the explicit determination of the deformation energy and related boundary conditions. The atypical features of the described continuum model for pantographic sheets are reflected by its atypical mathematical properties. That is:

- the PDEs determining the equilibrium configurations involve second and forthorder partial derivatives terms which may have a comparable order of magnitude; in the studied case of orthogonal fibers, the displacement fields along the fibers are governed by uncoupled PDEs;

- the deformation energy is not coercive in the standard sense. However, using specific boundary conditions, consistently established from the found expression for deformation energy, we establish the definite positiveness of the deformation energy functional, which simultaneously involves first- and secondorder of displacement gradients; we conjecture that such formulated problems are well-posed.

The corresponding atypical physical properties of pantographic sheets consist in the following circumstances:

- the extensional forces along a fiber are balanced by the shear forces due to the bending of its transverse fibers (see (44), (45)), 
- the balance of couples is separately valid for the two arrays of orthogonal fibers, so that the fiber rotation is proportional to the axial gradient of transverse displacement; see (46).

The numerical simulations which we have presented are aimed at illustrating the performances of the obtained model. Indeed, pantographic sheets show very peculiar deformation patterns exhibiting regions of concentrated deformation energy. In particular, when applied to rectangular specimen the model:

- it allows for the determination, already in the linear case, of the region where the deformation energy is localized without any further a priori assumptions;

- it shows that the diffusion patterns of deformation inside the specimen differ notably from those shown in first gradient material. In particular, they are canalized in a way determined by the material symmetry and boundary conditions, while their thickness is determined by the characteristic length specified by the competition between the first and second gradient stiffnesses;

- the described features are confirmed by considering a specimen of circular shape with rectangular holes.

All these features and predictions match at least qualitatively the experimental evidence, e.g., [dell'Isola et al. 2015b]. It then appears that the pantographic sheet is an archetypical-oriented material in which second gradient effect plays a crucial role and for which it is possible to get a close description of the underlying actual physical mechanism.

There are many worthy issues that may be investigated concerning the complex structure here analyzed:

- a linearized analysis of pantographic structures with nonorthogonal and uneven fibers (this case is also relevant as it may give a tangent model for large deformations) and the determination of some analytical solutions of found PDEs;

- the comparison of the presented theory with further experimental evidence for possible improvement in the idealized model;

- the exploration of the application of the model to the mechanics of fabrics, the analysis of the dissipation that can arise from the interaction of the fibers or from internal friction (see, e.g., [Nadler and Steigmann 2003]) and the extension to three-dimensional deformations;

- buckling phenomena which can produce wrinkling (see, e.g., [Giorgio et al. 2016; Rizzi and Varano 2011; Rizzi et al. 2013; Gabriele et al. 2012; Carassale and Piccardo 2010; Piccardo et al. 2015]) and damage detection and its evolution (see, e.g., [Yang et al. 2011; Misra and Singh 2013; Andreaus and Casini 2016; Placidi 2015; Placidi 2016]). 
Finally, as future and more challenging lines develop, considering biological applications, it is conceivable that a morphoelastic pantographic continuum can be employed as a "smart" prosthesis (see, e.g., [McMahon et al. 2011]). In this context, it is possible also to equip the pantographic structure with transducers that can act both as sensors or actuators in order to induce some functional adaptation capabilities (see, e.g., [D'Annibale et al. 2015a; D'Annibale et al. 2015b; Andreaus and Porfiri 2007]).

\section{Acknowledgements}

Claude Boutin gratefully acknowledges the Università di Roma La Sapienza for a visiting grant. The authors thank Tomasz Lekszycki, Marek Pawlikowski and Roman Grygoruk for having made Figure 2 available.

\section{References}

[Abdiwi et al. 2013] F. Abdiwi, P. Harrison, and W. R. Yu, "Modelling the shear-tension coupling of woven engineering fabrics", Adv. Materials Sci. Eng. 2013 (2013), art. id. 786769, 9 pp.

[Alibert and Della Corte 2015] J.-J. Alibert and A. Della Corte, "Second-gradient continua as homogenized limit of pantographic microstructured plates: a rigorous proof”, Z. Angew. Math. Phys. 66:5 (2015), 2855-2870.

[Alibert et al. 2003] J.-J. Alibert, P. Seppecher, and F. dell'Isola, "Truss modular beams with deformation energy depending on higher displacement gradients", Math. Mech. Solids 8:1 (2003), $51-73$.

[Allaire 1992] G. Allaire, "Homogenization and two-scale convergence", SIAM J. Math. Anal. 23:6 (1992), 1482-1518.

[Altenbach et al. 2011] H. Altenbach, V. A. Eremeyev, and L. P. Lebedev, "Micropolar shells as two-dimensional generalized continua models", pp. 23-55 in Mechanics of generalized continua, edited by H. Altenbach et al., Adv. Struct. Mater. 7, Springer, Heidelberg, 2011.

[Andreaus and Casini 2016] U. Andreaus and P. Casini, "Identification of multiple open and fatigue cracks in beam-like structures using wavelets on deflection signals", Contin. Mech. Thermodyn. 28:1-2 (2016), 361-378.

[Andreaus and Porfiri 2007] U. Andreaus and M. Porfiri, "Effect of electrical uncertainties on resonant piezoelectric shunting”, J. Intelligent Mat. Syst. Struct. 18:5 (2007), 477-485.

[Archimedes/Heath 1897] Archimedes, "On floating bodies", pp. 253-300 in The works of Archimedes, edited by T. L. Heath, Cambridge Univ. Press, 1897.

[Archimedes/Heath 1912] Archimedes, The method of Archimedes, recently discovered by Heiberg: a supplement to the works of Archimedes, 1897, edited by T. Heath, Cambridge Univ. Press, 1912.

[Auriault et al. 2009] J.-L. Auriault, C. Boutin, and C. Geindreau, Homogenization of coupled phenomena in heterogenous media, Wiley, Hoboken, NJ, 2009.

[Beirão da Veiga et al. 2008] L. Beirão da Veiga, J. Niiranen, and R. Stenberg, "A family of $C^{0}$ finite elements for Kirchhoff plates, II: Numerical results", Comput. Methods Appl. Mech. Engrg. 197:21-24 (2008), 1850-1864.

[Bensoussan et al. 2011] A. Bensoussan, J.-L. Lions, and G. Papanicolaou, Asymptotic analysis for periodic structures, AMS, Providence, RI, 2011. 
[Bîrsan et al. 2012] M. Bîrsan, H. Altenbach, T. Sadowski, V. A. Eremeyev, and D. Pietras, "Deformation analysis of functionally graded beams by the direct approach", Compos. B: Engrg. 43:3 (2012), 1315-1328.

[Boutin and Auriault 1993] C. Boutin and J.-L. Auriault, "Rayleigh scattering in elastic composite materials", Internat. J. Engrg. Sci. 31:12 (1993), 1669-1689.

[Boutin and Hans 2003] C. Boutin and S. Hans, "Homogenisation of periodic discrete medium: application to dynamics of framed structures”, Comp. Geotech. 30:4 (2003), 303-320.

[Boutin and Soubestre 2011] C. Boutin and J. Soubestre, "Generalized inner bending continua for linear fiber reinforced materials", Int. J. Solids Structures 48:3-4 (2011), 517-534.

[Boutin et al. 2010] C. Boutin, S. Hans, and C. Chesnais, "Generalized beams and continua: dynamics of reticulated structures", pp. 131-141 in Mechanics of generalized continua, edited by G. A. Maugin and A. V. Metrikine, Adv. Mech. Math. 21, Springer, New York, 2010.

[Caillerie 1984] D. Caillerie, "Thin elastic and periodic plates", Math. Methods Appl. Sci. 6:2 (1984), 159-191.

[Camar-Eddine and Seppecher 2001] M. Camar-Eddine and P. Seppecher, "Non-local interactions resulting from the homogenization of a linear diffusive medium", C. R. Acad. Sci. Paris Sér. I Math. 332:5 (2001), 485-490.

[Cao et al. 2008] J. Cao, R. Akkerman, P. Boisse, J. Chen, H. S. Cheng, E. F. de Graaf, J. L. Gorczyca, P. Harrison, G. Hivet, J. Launay, W. Lee, L. Liu, S. V. Lomov, A. Long, E. de Luycker, F. Morestin, J. Padvoiskis, X. Q. Peng, J. Sherwood, Tz. Stoilova, X. M. Tao, I. Verpoest, A. Willems, J. Wiggers, T. X. Yu, and B. Zhu, "Characterization of mechanical behavior of woven fabrics: experimental methods and benchmark results", Compos. A: Appl. Sci. Manuf. 39:6 (2008), 1037-1053.

[Carassale and Piccardo 2010] L. Carassale and G. Piccardo, "Non-linear discrete models for the stochastic analysis of cables in turbulent wind", Int. J. Non-Linear Mech. 45:3 (2010), 219-231.

[Carcaterra et al. 2015] A. Carcaterra, F. dell'Isola, R. Esposito, and M. Pulvirenti, "Macroscopic description of microscopically strongly inhomogeneous systems: a mathematical basis for the synthesis of higher gradients metamaterials", Arch. Ration. Mech. Anal. 218:3 (2015), 1239-1262.

[Casal 1966] P. Casal, "Principes variationnels en fluide compressible et en magnétodynamique des fluides", J. Méc., Paris 5 (1966), 149-161.

[Cazzani et al. 2016a] A. Cazzani, M. Malagù, and E. Turco, "Isogeometric analysis of plane-curved beams", Math. Mech. Solids 21:5 (2016), 562-577.

[Cazzani et al. 2016b] A. Cazzani, M. Malagù, E. Turco, and F. Stochino, "Constitutive models for strongly curved beams in the frame of isogeometric analysis", Math. Mech. Solids 21:2 (2016), 182-209.

[Cecchi and Rizzi 2001] A. Cecchi and N. L. Rizzi, "Heterogeneous elastic solids: a mixed homogenization-rigidification technique”, Internat. J. Solids Structures 38:1 (2001), 29-36.

[Chesnais et al. 2015] C. Chesnais, C. Boutin, and S. Hans, "Wave propagation and non-local effects in periodic frame materials: generalized continuum mechanics", Math. Mech. Solids 20:8 (2015), 929-958.

[d'Agostino et al. 2015] M. V. d'Agostino, I. Giorgio, L. Greco, A. Madeo, and P. Boisse, "Continuum and discrete models for structures including (quasi-)inexstensible elasticae with a view to the design and modeling of composite reinforcements", Int. J. Solids Structures 59 (2015), 1-17.

[D'Annibale et al. 2015a] F. D'Annibale, G. Rosi, and A. Luongo, "Linear stability of piezoelectriccontrolled discrete mechanical systems under nonconservative positional forces", Meccanica 50:3 (2015), 825-839. 
[D'Annibale et al. 2015b] F. D' Annibale, G. Rosi, and A. Luongo, "On the failure of the 'similar piezoelectric control' in preventing loss of stability by nonconservative positional forces", Z. Angew. Math. Phys. 66:4 (2015), 1949-1968.

[Del Vescovo and Giorgio 2014] D. Del Vescovo and I. Giorgio, "Dynamic problems for metamaterials: review of existing models and ideas for further research", Internat. J. Engrg. Sci. 80 (2014), 153-172.

[Della Corte et al. 2016] A. Della Corte, A. Battista, and F. dell'Isola, "Referential description of the evolution of a 2D swarm of robots interacting with the closer neighbors: perspectives of continuum modeling via higher gradient continua”, Int. J. Non-Linear Mech. 80 (2016), 209-220.

[dell'Isola and Placidi 2011] F. dell'Isola and L. Placidi, "Variational principles are a powerful tool also for formulating field theories", pp. 1-15 in Variational models and methods in solid and fluid mechanics, edited by F. dell'Isola and S. Gavrilyuk, CISM Courses and Lect. 535, Springer, Vienna, 2011.

[dell'Isola and Steigmann 2015] F. dell'Isola and D. Steigmann, "A two-dimensional gradient-elasticity theory for woven fabrics", J. Elasticity 118:1 (2015), 113-125.

[dell'Isola et al. 2009] F. dell'Isola, A. Madeo, and P. Seppecher, "Boundary conditions at fluidpermeable interfaces in porous media: a variational approach", Internat. J. Solids Structures 46:17 (2009), 3150-3164.

[dell'Isola et al. 2012] F. dell'Isola, P. Seppecher, and A. Madeo, "How contact interactions may depend on the shape of Cauchy cuts in $N$ th gradient continua: approach ' $a$ la d'Alembert", $Z$. Angew. Math. Phys. 63:6 (2012), 1119-1141.

[dell'Isola et al. 2015a] F. dell'Isola, U. Andreaus, and L. Placidi, "At the origins and in the vanguard of peridynamics, non-local and higher-gradient continuum mechanics: an underestimated and still topical contribution of Gabrio Piola", Math. Mech. Solids 20:8 (2015), 887-928.

[dell'Isola et al. 2015b] F. dell'Isola, T. Lekszycki, M. Pawlikowski, R. Grygoruk, and L. Greco, "Designing a light fabric metamaterial being highly macroscopically tough under directional extension: first experimental evidence", Z. Angew. Math. Phys. 66:6 (2015), 3473-3498.

[dell' Isola et al. 2015c] F. dell'Isola, P. Seppecher, and A. Della Corte, "The postulations à la d'Alembert and $\grave{a}$ la Cauchy for higher gradient continuum theories are equivalent: a review of existing results", Proc. A. 471:2183 (2015), art. id. 20150415, 25 pp.

[dell'Isola et al. 2016a] F. dell'Isola, A. Della Corte, and I. Giorgio, "Higher-gradient continua: the legacy of Piola, Mindlin, Sedov and Toupin and some future research perspectives", 2016. To appear in Math. Mech. Solids.

[dell'Isola et al. 2016b] F. dell'Isola, A. Della Corte, I. Giorgio, and D. Scerrato, "Pantographic 2D sheets: discussions of some numerical investigations and potential applications", Int. J. Non-Linear Mech. 80 (2016), 200-208.

[dell'Isola et al. 2016c] F. dell'Isola, A. Della Corte, L. Greco, and A. Luongo, "Plane bias extension test for a continuum with two inextensible families of fibres: a variational treatment with Lagrange multipliers and a perturbation design”, Int. J. Solids Structures 81 (2016), 1-12.

[dell'Isola et al. 2016d] F. dell'Isola, I. Giorgio, M. Pawlikowski, and N. L. Rizzi, "Large deformations of planar extensible beams and pantographic lattices: heuristic homogenization, experimental and numerical examples of equilibrium", Proc. Royal. Soc. A 472:2185 (2016), art. id. 20150790, $23 \mathrm{pp}$.

[dell'Isola et al. 2016e] F. dell'Isola, A. Madeo, and P. Seppecher, "Cauchy tetrahedron argument applied to higher contact interactions", Arch. Ration. Mech. Anal. 219:3 (2016), 1305-1341. 
[Dieudonné 1987] J. Dieudonné, Pour l'honneur de l'esprit humain: les mathématiques aujourd'hui, Librairie Hachette, Paris, 1987.

[Eremeyev 2016] V. A. Eremeyev, "On effective properties of materials at the nano- and microscales considering surface effects", Acta Mech. 227:1 (2016), 29-42.

[Eremeyev and Lebedev 2011] V. A. Eremeyev and L. P. Lebedev, "Existence theorems in the linear theory of micropolar shells", Z. Angew. Math. Mech. 91:6 (2011), 468-476.

[Federico and Grillo 2012] S. Federico and A. Grillo, "Elasticity and permeability of porous fibrereinforced materials under large deformations", Mech. Materials 44 (2012), 58-71.

[Gabriele et al. 2012] S. Gabriele, N. Rizzi, and V. Varano, "On the imperfection sensitivity of thinwalled frames", pp. 1-17, Paper 15 in Proceedings of the Eleventh International Conference on Computational Structures Technology, edited by B. H. V. Topping, Civil-Comp Proceedings 99, Civil-Comp Press, Slingshire, UK, 2012.

[Germain 1973] P. Germain, "The method of virtual power in continuum mechanics, II: Microstructure”, SIAM J. Appl. Math. 25:3 (1973), 556-575.

[Giorgio et al. 2015] I. Giorgio, R. Grygoruk, F. dell'Isola, and D. J. Steigmann, "Pattern formation in the three-dimensional deformations of fibered sheets", Mech. Res. Comm. 69 (2015), 164-171.

[Giorgio et al. 2016] I. Giorgio, A. Della Corte, F. dell'Isola, and D. J. Steigmann, "Buckling modes in pantographic lattices", Comptes Rendus Méc. 344:7 (2016), 487-501.

[Gouin and Debieve 1986] H. Gouin and J.-F. Debieve, "Variational principle involving the stress tensor in elastodynamics”, Int. J. Eng. Sci. 24:7 (1986).

[Greco and Cuomo 2013] L. Greco and M. Cuomo, "B-spline interpolation of Kirchhoff-Love space rods", Comput. Methods Appl. Mech. Engrg. 256 (2013), 251-269.

[Greco and Cuomo 2014] L. Greco and M. Cuomo, "An implicit $G^{1}$ multi patch B-spline interpolation for Kirchhoff-Love space rod”, Comput. Methods Appl. Mech. Engrg. 269 (2014), 173-197.

[Greco and Cuomo 2015] L. Greco and M. Cuomo, "Consistent tangent operator for an exact Kirchhoff rod model”, Contin. Mech. Thermodyn. 27:4 (2015), 861-877.

[Greco and Cuomo 2016] L. Greco and M. Cuomo, "An isogeometric implicit $G^{1}$ mixed finite element for Kirchhoff space rods", Comput. Methods Appl. Mech. Engrg. 298 (2016), 325-349.

[Grillo et al. 2015] A. Grillo, G. Wittum, A. Tomic, and S. Federico, "Remodelling in statistically oriented fibre-reinforced materials and biological tissues", Math. Mech. Solids 20:9 (2015), 11071129.

[Hans and Boutin 2008] S. Hans and C. Boutin, "Dynamics of discrete framed structures: a unified homogenized description”, J. Mech. Materials Struct. 3:9 (2008), 1709-1739.

[Harrison 2016] P. Harrison, "Modelling the forming mechanics of engineering fabrics using a mutually constrained pantographic beam and membrane mesh", Compos. A: Appl. Sci. Manuf. 81 (2016), $145-157$.

[Heath 1921a] Sir T. Heath, A history of Greek mathematics, I: From Thales to Euclid, Clarendon, Oxford, 1921.

[Heath 1921b] Sir T. Heath, A history of Greek mathematics, II: From Aristarchus to Diophantus, Clarendon, Oxford, 1921.

[Hero/Woodcroft 1851] B. Woodcroft (editor), The pneumatics of Hero of Alexandria, C. Whittingham, London, 1851.

[Launay et al. 2008] J. Launay, G. Hivet, A. V. Duong, and P. Boisse, "Experimental analysis of the influence of tensions on in plane shear behaviour of woven composite reinforcements", Composites Sci. Tech. 68:2 (2008), 506-515. 
[Lekszycki 1991] T. Lekszycki, "Application of variational methods in analysis and synthesis of viscoelastic continuous systems”, Mech. Struct. Machines 19:2 (1991), 163-192.

[Liew et al. 2000] J. Y. R. Liew, H. Chen, N. E. Shanmugam, and W. F. Chen, "Improved nonlinear plastic hinge analysis of space frame structures", Engrg. Struct. 22:10 (2000), 1324-1338.

[Madeo et al. 2015] A. Madeo, A. Della Corte, L. Greco, and P. Neff, "Wave propagation in pantographic 2D lattices with internal discontinuities”, Proc. Estonian Acad. Sci. 64:3S (2015), 325-330.

[McMahon et al. 2011] J. McMahon, A. Goriely, and M. Tabor, "Nonlinear morphoelastic plates, II: Exodus to buckled states", Math. Mech. Solids 16:8 (2011), 833-871.

[Melnik and Goriely 2013] A. V. Melnik and A. Goriely, "Dynamic fiber reorientation in a fiberreinforced hyperelastic material”, Math. Mech. Solids 18:6 (2013), 634-648.

[Mindlin 1964] R. D. Mindlin, "Micro-structure in linear elasticity", Arch. Rational Mech. Anal. 16:1 (1964), 51-78.

[Mindlin 1965] R. D. Mindlin, "Second gradient of strain and surface-tension in linear elasticity", Int. J. Solids Structures 1:4 (1965), 417-438.

[Misra and Singh 2013] A. Misra and V. Singh, "Micromechanical model for viscoelastic materials undergoing damage", Contin. Mech. Thermodyn. 25:2-4 (2013), 343-358.

[Nadler and Steigmann 2003] B. Nadler and D. J. Steigmann, "A model for frictional slip in woven fabrics", C. R. Méc. Acad. Sci. Paris 331:12 (2003), 797-804.

[Németh and Kocsis 2014] R. K. Németh and A. Kocsis, "Bielastic web of links: a discrete model of Csonka's beam", Int. J. Non-Linear Mech. 63 (2014), 49-59.

[Nikopour and Selvadurai 2014] H. Nikopour and A. P. S. Selvadurai, "Concentrated loading of a fibre-reinforced composite plate: experimental and computational modeling of boundary fixity", Compos. B: Engrg. 60 (2014), 297-305.

[Noor et al. 1978] A. K. Noor, M. S. Anderson, and W. H. Greene, "Continuum models for beamand platelike lattice structures", AIAA J. 16:12 (1978), 1219-1228.

[Piccardo et al. 2015] G. Piccardo, L. C. Pagnini, and F. Tubino, "Some research perspectives in galloping phenomena: critical conditions and post-critical behavior", Contin. Mech. Thermodyn. 27:1-2 (2015), 261-285.

[Pideri and Seppecher 1997] C. Pideri and P. Seppecher, "A second gradient material resulting from the homogenization of an heterogeneous linear elastic medium", Contin. Mech. Thermodyn. 9:5 (1997), 241-257.

[Piola 2014] G. Piola, The complete works of Gabrio Piola, I, edited by F. dell'Isola et al., Advanced Structured Materials 38, Springer, Cham, Switzerland, 2014.

[Pipkin 1980] A. C. Pipkin, "Some developments in the theory of inextensible networks", Quart. Appl. Math. 38:3 (1980), 343-355.

[Pipkin 1981] A. C. Pipkin, "Plane traction problems for inextensible networks", Quart. J. Mech. Appl. Math. 34:4 (1981), 415-429.

[Placidi 2015] L. Placidi, "A variational approach for a nonlinear 1-dimensional second gradient continuum damage model", Contin. Mech. Thermodyn. 27:4-5 (2015), 623-638.

[Placidi 2016] L. Placidi, "A variational approach for a nonlinear one-dimensional damage-elastoplastic second-gradient continuum model”, Contin. Mech. Thermodyn. 28:1-2 (2016), 119-137.

[Placidi et al. 2017] L. Placidi, U. Andreaus, and I. Giorgio, "Identification of two-dimensional pantographic structure via a linear D4 orthotropic second gradient elastic model", J. Eng. Math. 103:1 (2017), 1-21. 
[Rahali et al. 2015] Y. Rahali, I. Giorgio, J. F. Ganghoffer, and F. dell'Isola, "Homogenization à la Piola produces second gradient continuum models for linear pantographic lattices", Internat. J. Engrg. Sci. 97 (2015), 148-172.

[Rizzi and Varano 2011] N. L. Rizzi and V. Varano, "The effects of warping on the postbuckling behaviour of thin-walled structures”, Thin-Walled Struct. 49:9 (2011), 1091-1097.

[Rizzi et al. 2013] N. L. Rizzi, V. Varano, and S. Gabriele, "Initial postbuckling behavior of thinwalled frames under mode interaction”, Thin-Walled Struct. 68 (2013), 124-134.

[Russo 2004] L. Russo, The forgotten revolution: how science was born in 300 BC and why it had to be reborn, Springer, Berlin, 2004.

[Seppecher et al. 2011] P. Seppecher, J.-J. Alibert, and F. dell'Isola, "Linear elastic trusses leading to continua with exotic mechanical interactions", J. Phys: Conf. Series 319:1 (2011), art. id. 012018, $1-13$.

[Soubestre and Boutin 2012] J. Soubestre and C. Boutin, "Non-local dynamic behavior of linear fiber reinforced materials", Mech. Materials 55 (2012), 16-32.

[Steigmann and dell'Isola 2015] D. J. Steigmann and F. dell'Isola, "Mechanical response of fabric sheets to three-dimensional bending, twisting, and stretching", Acta Mech. Sin. 31:3 (2015), 373382 .

[Toupin 1964] R. A. Toupin, "Theories of elasticity with couple-stress", Arch. Rational Mech. Anal. 17:2 (1964), 85-112.

[Turco and Aristodemo 1998] E. Turco and M. Aristodemo, "A three-dimensional B-spline boundary element”, Comput. Methods Appl. Mech. Eng. 155:1-2 (1998), 119-128.

[Yang et al. 2011] Y. Yang, W. Y. Ching, and A. Misra, "Higher-order continuum theory applied to fracture simulation of nanoscale intergranular glassy film", J. Nanomech. Micromech. 1:2 (2011), $60-71$.

Received 9 Mar 2016. Revised 26 Oct 2016. Accepted 11 Jan 2017.

Claude Boutin: claude.boutin@entpe.fr

Département Génie Civil et Bâtiment - URA CNRS 1652, Ecole Nationale des Travaux Publics de l'Etat - Université de Lyon, rue Maurice Audin, 69518 Vaulx-en-Velin, France

FRANCESCO DELL'ISOLA: francesco.dellisola@uniroma1.it

Dept. di Ingegneria Strutturale e Geotecnica, Università di Roma "La Sapienza", Via Eudossiana 18, I-00184 Roma, Italy

IVAN GIORGIO: ivan.giorgio@uniroma1.it

Università di Roma "La Sapienza”, Via Eudossiana 18, I-00184 Roma, Italy

LUCA PLACIDI: luca.placidi@uninettunouniversity.net

International Telematic University Uninettuno, C.so Vittorio Emanuele II, 39, I-00186 Roma, Italy 


\title{
EXPLICIT FORMULAS FOR RELAXED DISARRANGEMENT DENSITIES ARISING FROM STRUCTURED DEFORMATIONS
}

\author{
Ana Cristina Barroso, José Matias, \\ MARCO MORANDOTTI AND DAVID R. OWEN
}

In this paper we derive explicit formulas for disarrangement densities of submacroscopic separations, switches, and interpenetrations in the context of firstorder structured deformations. Our derivation employs relaxation within one mathematical setting for structured deformations of a specific, purely interfacial density, and the formula we obtain agrees with one obtained earlier in a different setting for structured deformations. Coincidentally, our derivation provides an alternative method for obtaining the earlier result, and we establish new explicit formulas for other measures of disarrangements that are significant in applications.

1. Introduction

163

2. Proof of the upper-bound inequality 177

3. Aside on isotropic vectors 180

4. Completion of the proof of the upper-bound inequality 182

5. Additional explicit formulas for disarrangement densities 184

$\begin{array}{ll}\text { Acknowledgments } & 187\end{array}$

$\begin{array}{ll}\text { References } & 188\end{array}$

\section{Introduction}

Structured deformations provide a multiscale geometry that captures the contributions at the macrolevel of both smooth geometrical changes and nonsmooth geometrical changes (disarrangements) at submacroscopic levels. For each (first-order) structured deformation $(g, G)$ of a continuous body, the tensor field $G$ is known to be a measure of deformations without disarrangements, and $M:=\nabla g-G$ is known to be a measure of deformations due to disarrangements. The tensor fields $G$ and $M$ together deliver not only standard notions of plastic deformation, but $M$ and its curl deliver the Burgers vector field associated with closed curves in the body

\section{Communicated by Miroslav Šilhavý.}

MSC2010: primary 49J45; secondary 74A60, 74G65, 15A99.

Keywords: structured deformations, relaxation, disarrangements, interfacial density, bulk density, isotropic vectors. 
and the dislocation density field used in describing geometrical changes in bodies with defects. Recently, Owen and Paroni [2015] explicitly evaluated some relaxed energy densities arising in Choksi and Fonseca's [1997] energetics of structured deformations and thereby showed (1) $(\operatorname{tr} M)^{+}$, the positive part of $\operatorname{tr} M$, is a volume density of disarrangements due to submacroscopic separations, (2) $(\operatorname{tr} M)^{-}$, the negative part of $\operatorname{tr} M$, is a volume density of disarrangements due to submacroscopic switches and interpenetrations, and (3) $|\operatorname{tr} M|$, the absolute value of $\operatorname{tr} M$, is a volume density of all three of these nontangential disarrangements: separations, switches, and interpenetrations. The main contribution of the present research is to show that a different approach to the energetics of structured deformations, that due to Baía, Matias, and Santos [Baía et al. 2012], confirms the roles of $(\operatorname{tr} M)^{+}$, $(\operatorname{tr} M)^{-}$, and $|\operatorname{tr} M|$ established by Owen and Paroni. In doing so, we give an alternative, shorter proof of Owen and Paroni's results, and we establish additional explicit formulas for other measures of disarrangements.

To motivate our study and to provide necessary background, we briefly discuss in the following subsections of this introduction some concepts and results from the multiscale geometry of structured deformations. (Readers familiar with this material may wish to skip to Section 1E, where our main results are summarized.)

\section{A. Structured deformations and disarrangement densities in the setting of Del}

Piero and Owen. The need in continuum mechanics to include the effects of multiscale geometrical changes led Del Piero and Owen [1993] to a notion of structured deformations as triples $(\kappa, g, G)$, where

- the injective, piecewise continuously differentiable field $g$ maps the points of a continuous body into physical space and describes macroscopic changes in the geometry of the body,

- the piecewise continuous tensor field $G$ maps the body into the space of linear mappings on the translation space of physical space and satisfies the "accommodation inequality"

$$
0<C<\operatorname{det} G(x) \leq \operatorname{det} \nabla g(x) \quad \text { at each point } x,
$$

where $\nabla$ denotes the classical gradient operator, and

- $\kappa$ is a surface-like subset of the body that describes preexisting, unopened macroscopic cracks.

A geometrical interpretation of the field $G$ is provided by the approximation theorem [Del Piero and Owen 1993]: for each structured deformation $(\kappa, g, G)$, there exists a sequence of injective, piecewise smooth deformations $f_{n}$ and a sequence 
of surface-like subsets $\kappa_{n}$ of the body such that

$$
\begin{aligned}
g & =\lim _{n \rightarrow \infty} f_{n}, \\
G & =\lim _{n \rightarrow \infty} \nabla f_{n},
\end{aligned}
$$

and

$$
\kappa=\bigcup_{n=1}^{\infty} \bigcap_{p=n}^{\infty} \kappa_{p}
$$

The limits in (1-2) and (1-3) are taken in the sense of $L^{\infty}$ convergence. A sequence $n \mapsto f_{n}$ of piecewise smooth, injective functions satisfying (1-2) and (1-3) is called a determining sequence for the pair $(g, G)$, and each term $f_{n}$ is interpreted as describing the body divided into tiny pieces that may individually undergo smooth geometrical changes and that also may undergo disarrangements, i.e., may separate or slide relative to each other. In this context, we write $f_{n} \rightsquigarrow(g, G)$. From (1-3) we see that $G$ captures the effects at the macrolevel of smooth geometrical changes at submacroscopic levels, and we call $G$ the deformation without disarrangements.

Del Piero and Owen [1995] proved that for every structured deformation $(\kappa, g, G)$, for every determining sequence $n \mapsto f_{n}$ for $(g, G)$, and for every point $x$ where $g$ is differentiable and where $G$ is continuous there holds

$$
\lim _{r \rightarrow 0} \lim _{n \rightarrow \infty} \frac{\int_{J\left(f_{n}\right) \cap B_{r}(x)}\left[f_{n}\right](y) \otimes v(y) d \mathscr{H}^{N-1}(y)}{\left|B_{r}(x)\right|}=\nabla g(x)-G(x) .
$$

Here, $\mathscr{H}^{N-1}$ denotes the $(N-1)$-dimensional Hausdorff measure on $\mathbb{R}^{N}, B_{r}(x)$ denotes the open ball centered at $x$ of radius $r,\left|B_{r}(x)\right|$ denotes its volume (i.e., its $N$-dimensional Lebesgue measure), $J\left(f_{n}\right)$ denotes the jump set of $f_{n}$ (i.e., points where $f_{n}$ can suffer jump discontinuities), and $\left[f_{n}\right](y) \otimes v(y)$ is the tensor product of the jump [ $f_{n}$ ] of $f_{n}$ with the normal $v$ to the jump set. This result permits us to call the tensor

$$
M(x):=\nabla g(x)-G(x)
$$

the deformation due to disarrangements because it captures, in the limit as $n$ tends to infinity, the volume density of separations and slips between pieces of the body as described by the approximating deformations $f_{n}$. We may then regard the tensor field $M$ as a tensorial disarrangement density that, for every determining sequence $n \mapsto f_{n}$ for $(g, G)$, reflects the limits of interfacial discontinuities of the approximating deformations $f_{n}$. Moreover, (1-2) and (1-3) along with the definition of $M(1-5)$ yield the alternative formula for the disarrangement density:

$$
M=\nabla\left(\lim _{n \rightarrow \infty} f_{n}\right)-\lim _{n \rightarrow \infty} \nabla f_{n} .
$$


Consequently, $M$ quantitatively measures the lack of commutativity of the classical gradient $\nabla$ and the limit operator $\lim _{n \rightarrow \infty}$ for $L^{\infty}$ convergence.

The trivial algebraic relation

$$
\nabla g=G+M
$$

together with the identification relations (1-3) and (1-4) shows that the macroscopic deformation gradient $\nabla g$ has an additive decomposition into its part $G$ without disarrangements and its part $M$ due to disarrangements. Because $G$ has invertible values, (1-6) leads immediately to two multiplicative decompositions for $\nabla g$ :

$$
\nabla g=G\left(I+G^{-1} M\right)=\left(I+M G^{-1}\right) G .
$$

The disarrangement density $M$ and the deformation without disarrangements $G$ have an additional property significant in the description of defects and dislocations in a continuous body in three dimensions. We consider a smooth surface $\mathscr{S}$ with smooth bounding closed curve $\gamma$, both contained in a region in the body where $g$ and $G$ are smooth. The relation (1-6) and the smoothness of $g$ imply

$$
0=\oint_{\gamma} \nabla g(x) d x=\oint_{\gamma} G(x) d x+\oint_{\gamma} M(x) d x .
$$

The vector $\oint_{\gamma} M(x) d x$ measures the displacement due to disarrangements along $\gamma$ and may be called the Burgers vector [Del Piero and Owen 1993] for $\gamma$ arising from the given structured deformation. Application of Stokes' theorem to $\oint_{\gamma} G(x) d x$ and $\oint_{\gamma} M(x) d x$ and use of the previous relation yield the formulas for the Burgers vector:

$$
\oint_{\gamma} M(x) d x=\int_{\mathscr{S}} \operatorname{curl} M(x) v(x) d A_{x}=-\int_{\mathscr{S}} \operatorname{curl} G(x) v(x) d A_{x} .
$$

The second-order tensor field curl $M=-\operatorname{curl} G$ thus determines the Burgers vector associated with $\gamma$ for every closed curve and corresponds to familiar measures of dislocation density [Kröner 1958; Nye 1953]. In this manner, the disarrangement density tensor $M$ determines both the Burgers vector and the dislocation density tensor, both basic tools in modeling the effects of submacroscopic defects on the response of solids.

The tensorial relations (1-5) and (1-4) yield upon application of the trace operator the scalar relation

$$
\lim _{r \rightarrow 0} \lim _{n \rightarrow \infty} \frac{\int_{J\left(f_{n}\right) \cap B_{r}(x)}\left[f_{n}\right](y) \cdot v(y) d \mathscr{H}^{N-1}(y)}{\left|B_{r}(x)\right|}=\operatorname{tr} M(x)
$$

in which $\left[f_{n}\right](y) \cdot v(y)$ is the scalar product of the jump and of the normal at $y$. The formula (1-7) tells us that $\operatorname{tr} M$ is a scalar (bulk) disarrangement density that captures the components of the jumps of $f_{n}$ that are normal to the jump set. Moreover, 
this scalar disarrangement density at $x, \operatorname{tr} M(x)$, allows for cancellation of positive and negative contributions of $\left[f_{n}\right](y) \cdot v(y)$ at points $y$ near $x$ to the integral on the left-hand side of (1-7). Thus, $\operatorname{tr} M(x)$ does not distinguish between jumps with $\left[f_{n}\right](y) \cdot v(y)>0$ that pull apart small pieces of the body near $x$ and jumps with $\left[f_{n}\right](y) \cdot v(y)<0$ that cause small pieces near $x$ to switch places. Because the approximating $f_{n}$ are injective, the possibility for the case $\left[f_{n}\right](y) \cdot v(y)<0$ that $f_{n}$ can cause adjacent small pieces of the body to interpenetrate is ruled out.

Owen and Paroni [2015] refined the scalar disarrangement density $\operatorname{tr} M$ by replacing $\left[f_{n}\right](y) \cdot v(y)$ by its positive part throughout the jump set of $f_{n}$ or by its negative part throughout the jump set:

$$
\begin{aligned}
& \left(\left[f_{n}\right](y) \cdot v(y)\right)^{+}=\frac{1}{2}\left(\left|\left[f_{n}\right](y) \cdot v(y)\right|+\left[f_{n}\right](y) \cdot v(y)\right), \\
& \left(\left[f_{n}\right](y) \cdot v(y)\right)^{-}=\frac{1}{2}\left(\left|\left[f_{n}\right](y) \cdot v(y)\right|-\left[f_{n}\right](y) \cdot v(y)\right) .
\end{aligned}
$$

The field $\left(\left[f_{n}\right] \cdot v\right)^{+}$on the jump set is a scalar (interfacial) disarrangement density that measures separations of pieces of the body caused by $f_{n}$ while the field $\left(\left[f_{n}\right] \cdot v\right)^{-}$is a scalar (interfacial) disarrangement density that measures the switching of pieces of the body caused by $f_{n}$. Since

$$
\left|\left[f_{n}\right](y) \cdot v(y)\right|=\left(\left[f_{n}\right](y) \cdot v(y)\right)^{+}+\left(\left[f_{n}\right](y) \cdot v(y)\right)^{-},
$$

the field $\left|\left[f_{n}\right](y) \cdot v(y)\right|$ is a scalar disarrangement density that measures both separations and switches. We fix a part $\mathscr{P}$ of the body, integrate (1-8) or (1-9) over $J\left(f_{n}\right) \cap \mathscr{P}$, and use the formula (1-7) to obtain the relations

$$
\begin{aligned}
& \liminf _{n \rightarrow \infty} \int_{J\left(f_{n}\right) \cap \mathscr{P}}\left(\left[f_{n}\right](y) \cdot v(y)\right)^{ \pm} d \mathscr{H}^{N-1}(y) \\
& =\frac{1}{2} \liminf _{n \rightarrow \infty} \int_{J\left(f_{n}\right) \cap \mathscr{P}}\left|\left[f_{n}\right](y) \cdot v(y)\right| d \mathscr{H}^{N-1}(y) \\
& \quad \pm \frac{1}{2} \liminf _{n \rightarrow \infty} \int_{J\left(f_{n}\right) \cap \mathscr{P}}\left[f_{n}\right](y) \cdot v(y) d \mathscr{H}^{N-1}(y) \\
& =\frac{1}{2} \liminf _{n \rightarrow \infty} \int_{J\left(f_{n}\right) \cap \mathscr{P}}\left|\left[f_{n}\right](y) \cdot v(y)\right| d \mathscr{H}^{N-1}(y) \pm \frac{1}{2} \int_{\mathscr{P}} \operatorname{tr} M(x) d \mathscr{L}^{N}(x) .
\end{aligned}
$$

Consequently, the limiting behavior of the integral of $\left(\left[f_{n}\right](y) \cdot v(y)\right)^{ \pm}$in (1-10) as $n$ tends to $\infty$ is determined by the behavior of the integral of $\left|\left[f_{n}\right](y) \cdot v(y)\right|$, and we restrict our attention to the latter. We expect that

$$
\liminf _{n \rightarrow \infty} \int_{J\left(f_{n}\right) \cap \mathscr{P}}\left|\left[f_{n}\right](y) \cdot v(y)\right| d \mathscr{H}^{N-1}(y),
$$

unlike

$$
\liminf _{n \rightarrow \infty} \int_{J\left(f_{n}\right) \cap \Im}\left[f_{n}\right](y) \cdot v(y) d \mathscr{H}^{N-1}(y)
$$


will depend upon the choice of determining sequence for $(g, G)$. Therefore, we are led to consider the most economical manner in which separations and switches can arise among the determining sequences for $(g, G)$ :

$$
\begin{aligned}
\mathscr{V}^{|\cdot|}(g, G & ; \mathscr{P}) \\
& :=\inf \left\{\liminf _{n \rightarrow \infty} \int_{J\left(f_{n}\right) \cap \mathscr{P}}\left|\left[f_{n}\right](y) \cdot v(y)\right| d \mathscr{H}^{N-1}(y): f_{n} \rightsquigarrow(g, G)\right\} .
\end{aligned}
$$

The number $\mathscr{V}^{|\cdot|}(g, G ; \mathscr{P})$ so defined has the dimension of volume, and we call $\mathscr{V}^{l \cdot \mid}(g, G ; \mathscr{P})$ the (minimal) volume swept out by disarrangements in $\mathscr{P}$ for $(g, G)$. If we replace $|\cdot|$ everywhere in (1-11) by "+" or everywhere by "-", then we call the number $\mathscr{V}^{+}(g, G ; \mathscr{P})$ the (minimal) volume swept out by separations in $\mathscr{P}$ for $(g, G)$ and the number $\mathscr{V}^{-}(g, G ; \mathscr{P})$ the (minimal) volume swept out by switches in $\mathscr{P}$ for $(g, G)$. The formulas (1-10) imply the simple formulas

$$
\mathscr{V}^{ \pm}(g, G ; \mathscr{P})=\frac{1}{2} \mathscr{V}^{|\cdot|}(g, G ; \mathscr{P}) \pm \frac{1}{2} \int_{\mathscr{P}} \operatorname{tr} M(x) d \mathscr{L}^{N}(x)
$$

and, in view of the form of the second term on the right-hand side, raise the following basic question: does the volume swept out by disarrangements $\mathscr{V}^{|\cdot|}(g, G ; \mathscr{P})$ as defined in (1-11) have an associated disarrangement density which, when integrated over $\mathscr{P}$, recovers $\mathscr{V}^{\mid} \cdot \mid(g, G ; \mathscr{P})$ ? If so, what specific information can be obtained about the dependence of the integrand upon the structured deformation $(g, G)$ ?

While the setting for structured deformations described in this subsection is quite suitable for formulating refined field equations in continuum mechanics [Deseri and Owen 2003] that reflect the influence of submacroscopic geometrical changes in a body, this setting has not provided answers to questions such as the ones just raised. Part of the difficulty with the setting provided in [Del Piero and Owen 1993] lies in the choice of smoothness placed on $g$ and its approximates $f_{n}$ while another part lies in the requirement that $g$ and $f_{n}$ be injective. An alternative setting provided by Choksi and Fonseca [1997] was proposed for dealing with such questions and is described briefly in the next subsection.

\section{B. Structured deformations and disarrangement densities in Choksi and Fon-} seca's setting. We describe here a few essential elements of the treatment of structured deformations by Choksi and Fonseca [1997]. The articles [Choksi et al. 1999; Baía et al. 2012; 2011; Šilhavý 2015] also provide summaries of that treatment, and [Baía et al. 2012; 2011; Šilhavý 2015] provide alternative settings for structured deformations. The summary in [Choksi et al. 1999] is intended for those interested in immediate applications in continuum mechanics while [Baía et al. 2012] sets the stage for applications of structured deformations to thin bodies [Matias and Santos 2014]. The article [Šilhavý 2015] reexamines the results of [Choksi and 
Fonseca 1997] in a broader setting while providing refinements of counterparts of the approximation theorem and the identification relation (1-4).

According to Choksi and Fonseca, a structured deformation is a pair $(g, G)$ in which $g: \Omega \rightarrow \mathbb{R}^{N}$, with $\Omega$ an open subset of the space $\mathbb{R}^{N}$ of $N$-tuples of real numbers, and $G: \Omega \rightarrow \mathbb{R}^{N \times N}$, with $\mathbb{R}^{N \times N}$ the space of $N \times N$ matrices with real entries. The mapping $G$ is assumed to be integrable on $\Omega, G \in L^{1}\left(\Omega ; \mathbb{R}^{N \times N}\right)$, and $g$ is assumed to be in the space $S B V\left(\Omega ; \mathbb{R}^{N}\right)$, i.e., is a function of bounded variation with the additional property that its distributional derivative $D g$, as a bounded measure, has zero Cantor part:

$$
D g=\nabla g \mathscr{L}^{N}+[g] \otimes \nu \mathscr{H}^{N-1} .
$$

Here the integrable mapping $\nabla g$ is the density of the absolutely continuous part $\nabla g \mathscr{L}^{N}$ of $D g$ with respect to $N$-dimensional Lebesgue measure $\mathscr{L}^{N}$, and $[g] \otimes v$ is the density of the singular part $[g] \otimes \nu \mathcal{H}^{N-1}$ of $D g$ with respect to $(N-1)$ dimensional Hausdorff measure $\mathscr{H}^{N-1}$. The singular part is concentrated on $J(g)$, the jump set of $g$, and as usual, $[g]$ denotes the jump in $g$ and $v$ denotes the normal to the jump set $J(g)$. It is important to note that $\nabla g$ in the present setting is no longer the classical gradient of a smooth field and, consequently, need not be curlfree. Nevertheless, $\nabla g$ satisfies an integral version of the property of approximation by linear mappings that defines the classical gradient of smooth fields.

Choksi and Fonseca [1997] prove a version of the approximation theorem with approximating deformations $f_{n}$ also in $\operatorname{SBV}\left(\Omega ; \mathbb{R}^{N}\right)$ and with (1-2) and (1-3) replaced respectively by

$$
f_{n} \rightarrow g \quad \text { in } L^{1}\left(\Omega ; \mathbb{R}^{N}\right)
$$

and

$$
\nabla f_{n} \rightarrow G \quad \text { weakly in the sense of measures. }
$$

We note that no restriction in the form of the accommodation inequality (1-1) or in the form of a requirement of injectivity of $g$ or $f_{n}$ is imposed in the present context. We again use the term determining sequence to describe a sequence $n \mapsto f_{n}$ satisfying (1-13) and (1-14) for a given structured deformation $(g, G)$, and we again write $f_{n} \rightsquigarrow(g, G)$ when (1-13) and (1-14) both hold. The properties of distributional derivatives along with relations (1-12), (1-13), and (1-14) justify the calculation

$$
\begin{aligned}
\nabla g \mathscr{L}^{N}+[g] \otimes \nu \mathscr{H}^{N-1} & =D \lim _{n \rightarrow \infty} f_{n}=\lim _{n \rightarrow \infty} D f_{n} \\
& =\lim _{n \rightarrow \infty}\left(\nabla f_{n} \mathscr{L}^{N}+\left[f_{n}\right] \otimes \nu \mathcal{H}^{N-1}\right) \\
& =G \mathscr{L}^{N}+\lim _{n \rightarrow \infty}\left(\left[f_{n}\right] \otimes \nu \mathscr{H}^{N-1}\right),
\end{aligned}
$$

where the convergence indicated in the last three lines is weak convergence in the sense of measures. We conclude that the singular parts $\left[f_{n}\right] \otimes v \mathscr{H}^{N-1}$ of the 
approximating deformations $f_{n}$ converge in the same sense and that their limit satisfies

$$
\lim _{n \rightarrow \infty}\left(\left[f_{n}\right] \otimes v \mathscr{H}^{N-1}\right)=(\nabla g-G) \mathscr{L}^{N}+[g] \otimes \nu \mathscr{H}^{N-1} .
$$

In particular, the restriction of the limiting measure $\lim _{n \rightarrow \infty}\left(\left[f_{n}\right] \otimes v \mathscr{H}^{N-1}\right)$ to the complement of the jump set $J(g)$ agrees with the corresponding restriction of $(\nabla g-G) \mathscr{L}^{N}=M \mathscr{L}^{N}$. Consequently, the tensor field $M=\nabla g-G$ retains in this broader setting for structured deformations its identity as a tensor density of disarrangements for $(g, G)$. The formula (1-15) shows that when $M=\nabla g-G \neq 0$, while all of the measures $\left[f_{n}\right] \otimes v \mathscr{H}^{N-1}$ are supported on sets $J\left(f_{n}\right)$ of $\mathscr{L}^{N}$-measure zero and so have $\mathscr{L}^{N}$-parts zero, the limit measure $\lim _{n \rightarrow \infty}\left(\left[f_{n}\right] \otimes v \mathscr{H}^{N-1}\right)$ has $\mathscr{L}^{N}$-part $M \mathscr{L}^{N}$ nonzero. This observation points to the fact that the jump sets $J\left(f_{n}\right)$ can diffuse in the limit throughout the domain $\Omega$ so that the limiting measure $\lim _{n \rightarrow \infty}\left(\left[f_{n}\right] \otimes v \mathcal{H}^{N-1}\right)$ is supported in part on sets of positive $\mathscr{L}^{N}$-measure. This provides a counterpart in the $S B V$-setting to the relation (1-4) in which the limit of jumps on the left-hand side delivers the $\mathscr{L}^{N}$-density $M$. (See [Šilhavý 2015] for a detailed derivation of a counterpart of (1-4) in a somewhat broader setting for structured deformations than $S B V$.)

We briefly note that the scalar density of disarrangements $\operatorname{tr} M=\operatorname{tr}(\nabla g-G)$ that counts only normal components of jumps and that emerged in the previous setting also appears in the present setting when one takes the trace of every member of (1-15): if $f_{n} \rightsquigarrow(g, G)$, then

$$
\lim _{n \rightarrow \infty}\left(\left[f_{n}\right] \cdot v \mathscr{H}^{N-1}\right)=\operatorname{tr}(\nabla g-G) \mathscr{L}^{N}+[g] \cdot v \mathscr{H}^{N-1} .
$$

However, as was the case in the setting of Del Piero and Owen, replacement of $\left[f_{n}\right] \cdot v$ by $\left(\left[f_{n}\right] \cdot v\right)^{ \pm}$or by $\left|\left[f_{n}\right] \cdot v\right|$ need not yield a limit of the corresponding measures and, if a limit exists, the limit may depend upon the choice of determining sequence $n \mapsto f_{n}$. The setting of Choksi and Fonseca was formulated as a means of resolving these difficulties, and we summarize some aspects of that resolution in the next subsection.

1C. Relaxation of energies for structured deformations. In Section 1A we introduced the optimal function

$$
\mathscr{V}^{|\cdot|}(g, G ; \mathscr{P})=\inf \left\{\liminf _{n \rightarrow \infty} \int_{J\left(f_{n}\right) \cap \mathscr{P}}\left|\left[f_{n}\right](y) \cdot v(y)\right| d \mathscr{H}^{N-1}(y): f_{n} \rightsquigarrow(g, G)\right\} .
$$

Optimal functions arising from structured deformations can be analyzed using the results of Choksi and Fonseca [1997] on "relaxation of energies" for structured deformations. In that approach, the integral $\int_{J\left(f_{n}\right) \cap \mathscr{P}}\left|\left[f_{n}\right](y) \cdot v(y)\right| d \mathscr{H}^{N-1}(y)$ is 
replaced by an initial energy functional

$$
E\left(f_{n}\right)=\int_{\Omega} W\left(\nabla f_{n}(y)\right) d \mathscr{L}^{N}(y)+\int_{J\left(f_{n}\right) \cap \Omega} \psi\left(\left[f_{n}\right](y), v(y)\right) d \mathscr{H}^{N-1}(y)
$$

defined for $f_{n} \in S B V\left(\Omega ; \mathbb{R}^{N}\right)$. By imposing conditions on the initial bulk energy density $W$ and on the initial interfacial energy density $\psi$, the goal is to obtain for the relaxed energy $I(g, G)$ defined by

$$
\begin{aligned}
I(g, G):=\inf \left\{\liminf _{n \rightarrow \infty}(\right. & \int_{\Omega} W\left(\nabla f_{n}(y)\right) d \mathscr{L}^{N}(y) \\
& \left.\left.+\int_{J\left(f_{n}\right) \cap \Omega} \psi\left(\left[f_{n}\right](y), v(y)\right) d \mathscr{H}^{N-1}(y)\right): f_{n} \rightsquigarrow(g, G)\right\}
\end{aligned}
$$

a representation of the form

$$
I(g, G)=\int_{\Omega} H(\nabla g(y), G(y)) d \mathscr{L}^{N}(y)+\int_{J(g) \cap \Omega} h([g](y), v(y)) d \mathscr{H}^{N-1}(y)
$$

and to deduce properties of the relaxed bulk energy density $H$ and the relaxed interfacial energy density $h$. Because our present interest lies in the case of disarrangement densities, and not on the full energetics of structured deformations, we shall restrict our attention to the case $W=0$, and we record the following adaptation for the case $W=0$ of results from [Choksi and Fonseca 1997] (see [Owen and Paroni 2015, Theorem 3] for further comments and other adaptations).

Theorem 1.1. Let $S^{N-1}=\left\{v \in \mathbb{R}^{N}:|v|=1\right\}$. Let $\Omega$ be a bounded open subset of $\mathbb{R}^{N}$ and assume $\psi: \mathbb{R}^{N} \times S^{N-1} \rightarrow[0,+\infty)$ satisfies the following conditions:

(H1) There exists a constant $C>0$ such that, for all $(\xi, v) \in \mathbb{R}^{N} \times S^{N-1}$,

$$
0 \leq \psi(\xi, \nu) \leq C|\xi|
$$

(H2) $\psi(\cdot, v)$ is positively homogeneous of degree 1: for all $t>0$ and $(\xi, v) \in$ $\mathbb{R}^{N} \times S^{N-1}$, we have

$$
\psi(t \xi, v)=t \psi(\xi, v)
$$

(H3) $\psi(\cdot, v)$ is subadditive; i.e., for all $\xi_{1}, \xi_{2} \in \mathbb{R}^{N}$ and $v \in S^{N-1}$,

$$
\psi\left(\xi_{1}+\xi_{2}, v\right) \leq \psi\left(\xi_{1}, v\right)+\psi\left(\xi_{2}, v\right)
$$

Then, for any $p>1$, if we define 


$$
\begin{aligned}
& I(g, G):=\inf \left\{\liminf _{n \rightarrow \infty} \int_{J\left(u_{n}\right) \cap \Omega} \psi\left(\left[u_{n}\right], v\right) d \mathcal{H}^{N-1}:\right. \\
& u_{n} \in S B V\left(\Omega ; \mathbb{R}^{N}\right), u_{n} \rightarrow g \text { in } L^{1}\left(\Omega ; \mathbb{R}^{N}\right), \\
&\left.\nabla u_{n} \stackrel{*}{\rightarrow} G, \sup _{n}\left(\left|\nabla u_{n}\right|_{L^{p}\left(\Omega ; \mathbb{R}^{N \times N}\right)}+\left|D u_{n}\right|(\Omega)\right)<+\infty\right\},
\end{aligned}
$$

we have

$$
I(g, G)=\int_{\Omega} H(\nabla g(x), G(x)) d \mathscr{L}^{N}+\int_{J(g) \cap \Omega} h([g](x), v(x)) d \mathscr{H}^{N-1}(x),
$$

where

$$
\begin{aligned}
H(A, B):=\inf \left\{\int_{J(u) \cap Q} \psi([u], v) d \mathscr{H}^{N-1}:\right. & \\
& u \in S B V\left(Q ; \mathbb{R}^{N}\right),\left.u\right|_{\partial Q}=A x, \\
& \left.|\nabla u| \in L^{p}(Q), \int_{Q} \nabla u d \mathscr{L}^{N}=B\right\}
\end{aligned}
$$

and

$$
\begin{aligned}
h(\xi, \eta):=\inf \left\{\int_{J(u) \cap Q_{\eta}} \psi([u], v) d \mathscr{H}^{N-1}:\right. \\
\left.u \in \operatorname{SBV}\left(Q_{\eta} ; \mathbb{R}^{N}\right),\left.u\right|_{\partial Q_{\eta}}=u_{\xi, \eta}, \nabla u=0 \text { a.e. }\right\}
\end{aligned}
$$

with

$$
u_{\xi, \eta}(x):= \begin{cases}0 & \text { if }-\frac{1}{2} \leq x \cdot \eta<0 \\ \xi & \text { if } \quad 0 \leq x \cdot \eta<\frac{1}{2}\end{cases}
$$

Here, $Q=\left(-\frac{1}{2}, \frac{1}{2}\right)^{N}$ and $Q_{\eta}$ denotes the unit cube centered at the origin and with two faces normal to $\eta$.

On the right side of (1-17), we have corrected an inconsequential misprint that is present in the corresponding formula in Theorem 3 of [Owen and Paroni 2015].

Another approach to relaxation of energies for structured deformations in the full $B V$ setting is provided in [Baía et al. 2012]. A structured deformation in [Baía et al. 2012] is a pair

where

$$
(g, G) \in B V^{2}\left(\Omega ; \mathbb{R}^{N}\right) \times B V\left(\Omega ; \mathbb{R}^{N \times N}\right),
$$

$$
B V^{2}\left(\Omega ; \mathbb{R}^{N}\right):=\left\{u \in B V\left(\Omega ; \mathbb{R}^{N}\right): \nabla u \in B V\left(\Omega ; \mathbb{R}^{N \times N}\right)\right\} .
$$

The counterpart of the approximation theorem in this context asserts that there exists a sequence $f_{n} \in B V^{2}\left(\Omega ; \mathbb{R}^{N}\right)$ such that both $f_{n} \rightarrow g$ and $\nabla f_{n} \rightarrow G$ in the $L^{1}$-norm. In this case, we write $f_{n} \rightsquigarrow(g, G)$. 
The energy functional considered in [Baía et al. 2012], under assumptions on the initial bulk and surface energy densities similar to the ones in [Choksi and Fonseca 1997], reads

$$
\begin{aligned}
E\left(f_{n}\right)=\int_{\Omega} W\left(\nabla f_{n}(y), \nabla^{2} f_{n}(y)\right) d \mathscr{L}^{N} y & +\int_{J\left(f_{n}\right)} \psi\left(\left[f_{n}\right](y), v(y)\right) d \mathscr{H}^{N-1}(y) \\
& +\int_{J\left(\nabla f_{n}\right)} \psi_{1}\left(\left[\nabla f_{n}\right](y), v(y)\right) d \mathscr{H}^{N-1}(y),
\end{aligned}
$$

and the relaxed energy $I(g, G)$ is defined by

$$
I(g, G):=\inf \left\{\liminf _{n \rightarrow \infty} E\left(f_{n}\right): f_{n} \rightsquigarrow(g, G)\right\} .
$$

A crucial result in [Baía et al. 2012] is that (1-19) can be divided into two first-order relaxed energies, namely, $I(g, G)=I_{1}(g, G)+I_{2}(G)$, where the term $I_{1}(g, G)$ captures the structured deformation whereas $I_{2}(G)$ only depends on the deformation without disarrangements $G$. In the relevant case for the present paper, i.e., $W=\psi_{1}=0$, the results in [Baía et al. 2012] give $I_{2}=0$ and

$$
I_{1}(g, G):=\inf \left\{\liminf _{n \rightarrow \infty} \int_{J\left(f_{n}\right) \cap \Omega} \psi\left(\left[f_{n}\right](y), v(y)\right) d \mathscr{H}^{N-1}(y): f_{n} \rightsquigarrow(g, G)\right\} .
$$

Defining $S B V^{2}\left(\Omega ; \mathbb{R}^{N}\right):=\left\{u \in S B V\left(\Omega ; \mathbb{R}^{N}\right): \nabla u \in S B V\left(\Omega ; \mathbb{R}^{N \times N}\right)\right\}$, the following representation theorem holds

Theorem 1.2 [Baía et al. 2012, Theorem 3.2]. For every $(g, G) \in S B V^{2}\left(\Omega ; \mathbb{R}^{N}\right) \times$ $\operatorname{SBV}\left(\Omega ; \mathbb{R}^{N \times N}\right)$, given $\psi$ under the same hypotheses $(\mathrm{H} 1)-(\mathrm{H} 3)$ of Theorem 1.1,

$$
I(g, G)=\int_{\Omega} H(G(x)-\nabla g(x)) d \mathscr{L}^{N}+\int_{J(g) \cap \Omega} h([g](x), v(x)) d \mathscr{H}^{N-1}(x),
$$

where, given $A \in \mathbb{R}^{N \times N}, \xi \in \mathbb{R}^{N}$, and $\eta \in S^{N-1}$,

$$
\begin{aligned}
& H(A):=\inf \left\{\int_{J(u) \cap Q} \psi([u], v) d \mathscr{H}^{N-1}:\right. \\
& \left.u \in S B V^{2}\left(Q ; \mathbb{R}^{N}\right),\left.u\right|_{\partial Q}=0, \nabla u=A \text { a.e. in } Q\right\}
\end{aligned}
$$

and

$$
\begin{aligned}
h(\xi, \eta):=\inf \left\{\int_{J(u) \cap Q_{\eta}} \psi([u], v) d \mathcal{H}^{N-1}:\right. \\
\left.u \in S B V^{2}\left(Q_{\eta} ; \mathbb{R}^{N}\right),\left.u\right|_{\partial Q_{\eta}}=u_{\xi, \eta}, \nabla u=0 \text { a.e. in } Q\right\},
\end{aligned}
$$

with $u_{\xi, \eta}$ defined as in (1-18). 
Remark 1.3. It is worth noticing that the minimum problems defining (1-20) and (1-21) are formally performed in $S B V^{2}\left(\Omega ; \mathbb{R}^{N}\right)$, but the result is the same if $S B V^{2}$ is replaced in these relations by $S B V$, due to the requirement that $\nabla u$ be constant.

1D. Explicit formulas for relaxed disarrangement densities. Owen and Paroni [2015] applied Theorem 1.1 to the specific disarrangement densities $\left|\left[f_{n}\right](y) \cdot v(y)\right|$ and $\left(\left[f_{n}\right](y) \cdot v(y)\right)^{ \pm}$introduced in Section 1A and obtained for each of these densities an explicit formula for the corresponding relaxed disarrangement densities $H$ in (1-16) and $h$ in (1-17). Among their results [Owen and Paroni 2015, Theorem 4] is the following (obtained by setting $L(x)=I$ in their Theorem 4):

Theorem 1.4. The initial disarrangement densities

$$
\begin{aligned}
\psi^{|\cdot|}(\xi, v) & :=|\xi \cdot v|, \\
\psi^{ \pm}(\xi, v) & :=(\xi \cdot v)^{ \pm}
\end{aligned}
$$

satisfy the hypotheses $(\mathrm{H1})-(\mathrm{H} 3)$ in Theorem 1.1 and have relaxed disarrangement densities given by

$$
\begin{aligned}
H^{|\cdot|}(A, B) & =|\operatorname{tr}(A-B)|, & h^{|\cdot|}(\xi, v) & =|\xi \cdot v|=\psi^{|\cdot|}(\xi, v), \\
H^{ \pm}(A, B) & =(\operatorname{tr}(A-B))^{ \pm}, & h^{ \pm}(\xi, v) & =(\xi \cdot v)^{ \pm}=\psi^{ \pm}(\xi, v) .
\end{aligned}
$$

Specifically, when the minimal volume that is swept out by disarrangements $\mathscr{V}^{|\cdot|}(g, G ; \mathscr{P})$ is defined in the Choksi-Fonseca setting by (1-11), then (1-23) yields the explicit formula

$$
\begin{aligned}
& \mathscr{V}^{|\cdot|}(g, G ; \mathscr{P}) \\
& \quad=\int_{\mathscr{P}}|\operatorname{tr}(\nabla g(x)-G(x))| d \mathscr{L}^{N}(x)+\int_{J(g) \cap \mathscr{P}}|[g](x) \cdot v(x)| d \mathscr{H}^{N-1}(x)
\end{aligned}
$$

for the (minimal) volume swept out by separations and switches among approximations $f_{n}$ that determine $(g, G)$. Relation (1-24) provides answers in the setting of Choksi and Fonseca to the questions raised at the end of Section 1A: ${ }^{\mathscr{V}}|\cdot|(g, G ; \mathscr{P})$ has both a bulk disarrangement density $|\operatorname{tr}(\nabla g-G)|=|\operatorname{tr} M|$ and an interfacial disarrangement density $|[g] \cdot v|$. Similarly, Theorem 1.4 shows that the (minimal) volume swept out by separations alone, $\mathscr{V}^{+}(g, G ; \mathscr{P})$, has the bulk disarrangement density $(\operatorname{tr} M)^{+}$and the interfacial disarrangement density $([g] \cdot v)^{+}$, with a corresponding result for $\mathscr{V}^{-}(g, G ; \mathscr{P})$, the (minimal) volume swept out by switches and interpenetrations (the approximations $f_{n}$ in the Choksi-Fonseca setting are not required to be injective so that interpenetrations can arise there, unlike in the setting of Del Piero-Owen). 
1E. Summary of the research presented in the present article. In the proof of Theorem 1.4 given in [Owen and Paroni 2015], the significant part of the argument addresses the verification of the inequality

$$
H^{|\cdot|}(A, B) \leq|\operatorname{tr}(A-B)|,
$$

where $H^{|\cdot|}(A, B)$ is given by the right-hand side of (1-16) with $\psi\left([u], v_{u}\right)$ replaced by $\psi^{|\cdot|}([u], v)=|[u] \cdot v|$. This inequality was proved in [Owen and Paroni 2015] by constructing a family $u_{\varepsilon}$ of piecewise affine mappings on the unit cube $Q$, each of whose jump set $J\left(u_{\varepsilon}\right)$ is formed by two (planar) ends and by a lateral surface constructed from solution curves of the differential equation $\dot{x}=(A-B) x$. The lateral surface, by construction, contributes nothing to $\int_{J(u) \cap Q_{\eta}}|[u] \cdot v| d \mathscr{H}^{N-1}$, and the contributions of the two ends can be calculated explicitly for $A-B$ lying in a dense subset of $\mathbb{R}^{N \times N}$. Proposition 5.2 of [Choksi and Fonseca 1997] provides sufficient regularity of $H^{|\cdot|}(A, B)$ to establish (1-25) for all $A-B \in \mathbb{R}^{N \times N}$.

As one of the main results in this article, we provide an alternate, shorter proof of (1-25) that employs a different family $u_{\varepsilon}$ of piecewise affine mappings that does not involve solution curves of $\dot{x}=(A-B) x$. Our approach is based on the following observation. With $A, B \in \mathbb{R}^{N \times N}, p>1$, and $Q=\left(-\frac{1}{2}, \frac{1}{2}\right)^{N}$,

$$
\begin{gathered}
|\operatorname{tr}(A-B)| \leq \inf \left\{\int_{J(u)}|[u](x) \cdot v(x)| d \mathscr{H}^{N-1}(x): u \in S B V\left(Q ; \mathbb{R}^{N}\right),\right. \\
\left.u(x)=A x \text { on } \partial Q, \nabla u \in L^{p}(Q), \int_{Q} \nabla u(x) d \mathscr{L}^{N}(x)=B\right\} \\
\leq \inf \left\{\int_{J(u)}|[u](x) \cdot v(x)| d \mathscr{H}^{N-1}(x): u \in S B V\left(Q ; \mathbb{R}^{N}\right),\right. \\
u(x)=0 \text { on } \partial Q, \nabla u=B-A \text { a.e. }\} .
\end{gathered}
$$

The first inequality follows by moving the absolute value outside the integral and using the Gauss-Green theorem for the space $S B V\left(Q ; \mathbb{R}^{N}\right)$ of special functions of bounded variation while the second follows by noting that, if $u$ satisfies the last set of conditions, then the function $x \mapsto u(x)+A x$ satisfies the first set of conditions. In this paper, we wish to show that

$$
\begin{aligned}
\inf \left\{\int_{J(u)}|[u](x) \cdot v(x)| d \mathscr{H}^{N-1}(x): u \in S B V\left(Q ; \mathbb{R}^{N}\right),\right. \\
u(x)=0 \text { on } \partial Q, \nabla u=B-A \text { a.e. }\} \leq|\operatorname{tr}(A-B)|
\end{aligned}
$$

so that the two infima in (1-26) have common value $|\operatorname{tr}(A-B)|$.

The second main contribution of the present research concerns the alternative approach to structured deformations and to relaxed energies due to Baía, Matias, 
and Santos [Baía et al. 2012] discussed at the end of Section 1C. According to that discussion, the second infimum in (1-26) (see (1-20) and Remark 1.3)

$$
\begin{aligned}
& \inf \left\{\int_{J(u)}|[u](x) \cdot v(x)| d \mathscr{H}^{N-1}(x):\right. \\
& \left.\qquad u \in \operatorname{SBV}\left(Q ; R^{N}\right), u(x)=0 \text { on } \partial Q, \nabla u=B-A \text { a.e. in } Q\right\}
\end{aligned}
$$

is the bulk disarrangement density for the same interfacial disarrangement density $\psi^{|\cdot|}([u], v)(1-22)$ studied by Owen and Paroni in the setting of Choksi and Fonseca. Consequently, our proof of (1-27) establishes the equality of the bulk disarrangement densities obtained in two different settings for structured deformations. Thus, the geometrical significance of the expression $|\operatorname{tr}(A-B)|$ described in [Owen and Paroni 2015], namely, a volume density of volume swept out by nonsmooth, submacroscopic geometrical changes, is strengthened by the fact that the same expression arises from two different schemes of relaxation. We note that the two different schemes of relaxation also deliver the same formula for the (relaxed) interfacial disarrangement density $h: h=\psi^{|\cdot|}$ (see [Owen and Paroni 2015] for the routine verification that applies to both schemes).

The explicit formulas for disarrangement densities considered here in the context of structured deformations will provide scalar fields that can enter as variables in constitutive relations for the response of three-dimensional bodies. For this purpose, frame-indifferent variants of the specific fields obtained here are available through known factorizations of structured deformations in which the factor that tracks disarrangements is unchanged under changes in frame [Del Piero and Owen 1993]. Our explicit formulas are also starting points for the study of examples in other contexts involving structured deformations: second-order structured deformations [Owen and Paroni 2000] in which second gradients and their limits enter into submacroscopic changes in geometry as well as processes for dimension reduction [Matias and Santos 2014] in the presence of disarrangements that describe thin structures undergoing submacroscopic slips, separations, and switches.

In Section 2, we provide a "tilted cube" construction for the family $u_{\varepsilon}$ of functions employed in proving (1-27). The common orientation of the tilted cubes is determined in Section 3 by means of a known result on the isotropic vectors of symmetric linear mappings. The proof of (1-27) is completed in Section 4, and the paper concludes with Section 5 with some additional explicit formulas for disarrangement densities.

During the review of this article, the research of [Šilhavý 2016] was brought to our attention, in which explicit formulas for the bulk and interfacial relaxed energies are established for a broad class of purely interfacial initial energies that includes the ones studied here. 


\section{Proof of the upper-bound inequality}

In what follows, a proof of (1-27) is given. The proof requires the following instance of Lemma 4.3 in [Matias 2007].

Lemma 2.1. Let $M \in \mathbb{R}^{N \times N}$ and a bounded open set $\Omega \subset \mathbb{R}^{N}$ be given, with $\Omega$ having Lipshitz boundary. There exist a number $C(N)>0$, independent of $M$ and $\Omega$, and $u \in \operatorname{SBV}\left(\Omega ; \mathbb{R}^{N}\right)$ such that

(1) $\left.u\right|_{\partial \Omega}=0$,

(2) $\nabla u=M, \mathscr{L}^{N}$-a.e. on $\Omega$, and

(3) $\left|D^{s} u\right|(\Omega) \leq C(N)\|M\| \mathscr{L}^{N}(\Omega)$.

Here, $\nabla u$ and $D^{s} u$ denote the absolutely continuous and the singular parts of the distributional derivative $D u=\nabla u \mathscr{L}^{N}+D^{s} u$ of $u$, and $\left|D^{s} u\right|$ denotes the total variation of the singular part. In addition, $\|M\|:=\left(\operatorname{tr}\left(M^{T} M\right)\right)^{1 / 2}$ is the Euclidean norm of the matrix $M$. We shall now use the lemma to verify (1-27) for $M=A-B$. To this end, let an integer $n \geq 1$ be given and consider the frame

$$
\mathscr{F}_{n}:=Q \backslash \overline{\left(1-\frac{2}{n+2}\right) Q} \text {. }
$$

We may apply the lemma to obtain an $S B V$ function $u^{(n)}: \mathscr{F}_{n} \rightarrow \mathbb{R}^{N}$ such that

- $\left.u^{(n)}\right|_{\partial \mathscr{F}_{n}}=0$,

- $\nabla u^{(n)}=M, \mathscr{L}^{N}$-a.e. on $\mathscr{F}_{n}$, and

- the total variation $\int_{J\left(u^{(n)}\right)}\left|\left[u^{(n)}\right]\right|(x) d \mathscr{H}^{N-1}(x)$ of $u^{(n)}$ satisfies

$$
\int_{J\left(u^{(n)}\right)}\left|\left[u^{(n)}\right]\right|(x) d \mathscr{H}^{N-1}(x) \leq C(N)\|M\|\left(1-\left(1-\frac{2}{n+2}\right)^{N}\right) .
$$

In preparation for defining an appropriate function $u$ on $Q \backslash \overline{\mathscr{F}_{n}}=\left(1-\frac{2}{n+2}\right) Q$, we write $\widehat{M}:=\frac{1}{2}\left(M+M^{T}\right)$ for the symmetric part of $M$, and we choose an orthonormal basis $e_{i}, i=1, \ldots, N$, of $\mathbb{R}^{N}$ that consists of eigenvectors of $\widehat{M}$ :

$$
\widehat{M} e_{i}=\lambda_{i} e_{i}, \quad i=1, \ldots, N .
$$

We let $m$ be a positive integer and cover $\left(1-\frac{2}{n+2}\right) Q$ by a collection $\mathscr{C}_{n, m}$ of congruent, nonoverlapping open cubes $C_{n, m}^{k}, k=1, \ldots, K_{n, m}$, each of edge-length $1 / m$ and each with the $i$-th pair of opposite faces orthogonal to the unit vector $R e_{i}$, for $i=1, \ldots, N$. Here, $R$ is an orthogonal $N \times N$ matrix, $R R^{T}=R^{T} R=I$, to be determined presently. We require in addition that each cube $C_{n, m}^{k}$ satisfies

$$
\left(1-\frac{2}{n+2}\right) Q \cap C_{n, m}^{k} \neq \varnothing \text {. }
$$


We denote by $c_{n, m}^{k}$ the center of $C_{n, m}^{k}$, and we define $u_{n, m}:\left(1-\frac{2}{n+2}\right) Q \rightarrow \mathbb{R}^{N}$ by $u_{n, m}(x):= \begin{cases}M\left(x-c_{n, m}^{k}\right) & \text { if } x \in\left(1-\frac{2}{n+2}\right) Q \cap C_{n, m}^{k} \text { for some } k=1, \ldots, K_{n, m}, \\ 0 & \text { if } x \in\left(1-\frac{2}{n+2}\right) Q \backslash \bigcup_{k=1}^{K_{n, m}} C_{n, m}^{k} .\end{cases}$

Using standard reasoning, we conclude that $u_{n, m} \in S B V\left(\left(1-\frac{2}{n+2}\right) Q ; \mathbb{R}^{N}\right)$ with $\nabla u_{n, m}=M, \mathscr{L}^{N}$-a.e. on $\left(1-\frac{2}{n+2}\right) Q$. Moreover, the trace of $u_{n, m}$ on $\partial\left(\left(1-\frac{2}{n+2}\right) Q\right)$ is bounded pointwise by $(\sqrt{N} / 2 m)\|M\|$. Consequently, the function $u_{m}^{(n)}: Q \rightarrow \mathbb{R}^{N}$ defined by

$$
u_{m}^{(n)}(x):= \begin{cases}u^{(n)}(x) & \text { for } x \in \mathscr{F}_{n}, \\ u_{n, m}(x) & \text { for } x \in\left(1-\frac{2}{n+2}\right) Q\end{cases}
$$

belongs to $\operatorname{SBV}\left(Q ; \mathbb{R}^{N}\right)$, has gradient $M, \mathscr{L}^{N}$-a.e., and has zero trace on $\partial Q$. Moreover, the jump set of $u_{m}^{(n)}$ satisfies

$$
J\left(u_{m}^{(n)}\right) \subset J\left(u^{(n)}\right) \cup \partial\left(\left(1-\frac{2}{n+2}\right) Q\right) \cup J\left(u_{n, m}\right) .
$$

Since $u_{m}^{(n)}$ has outer trace 0 on $\partial\left(1-\frac{2}{n+2}\right) Q$, for $\mathscr{H}^{N-1}$-a.e. $x$ in $\partial\left(\left(1-\frac{2}{n+2}\right) Q\right)$

$$
\left|\left[u_{m}^{(n)}\right](x)\right| \leq \frac{\sqrt{N}}{m}\|M\|
$$

and, consequently,

$$
\int_{\partial((1-2 /(n+2)) Q)}\left|\left[u_{m}^{(n)}\right](x) \cdot v(x)\right| d \mathscr{H}^{N-1}(x) \leq \frac{\sqrt{N}}{m}\|M\| 2 N\left(1-\frac{2}{n+2}\right)^{N-1} .
$$

We note from (2-1) that

$$
\int_{J\left(u^{(n)}\right)}\left|\left[u_{m}^{(n)}\right](x) \cdot v(x)\right| d \mathscr{H}^{N-1}(x) \leq C(N)\|M\|\left(1-\left(1-\frac{2}{n+2}\right)^{N}\right)
$$

and we seek a corresponding estimate for $\int_{J\left(u_{n, m}\right)}\left|\left[u_{m}^{(n)}\right](x) \cdot v(x)\right| d \mathscr{H}^{N-1}(x)$. To this end, we note that

$$
J\left(u_{n, m}\right) \subset \bigcup_{k=1}^{K_{n, m}} \partial C_{n, m}^{k},
$$

and we shall seek an upper bound for $\int_{\bigcup_{k=1}^{K n, m} \partial C_{n, m}^{k}}\left|\left[u_{m}^{(n)}\right](x) \cdot v(x)\right| d \mathscr{H}^{N-1}(x)$. For each $k=1, \ldots, K_{n, m}$ and $i=1, \ldots, N$, we denote by $\phi_{n, m}^{k, i+}$ and $\phi_{n, m}^{k, i-}$ the two faces of the cube $C_{n, m}^{k} \in \mathscr{C}_{n, m}$ orthogonal to $R e_{i}$. We note that one face $\phi_{n, m}^{k, i+}$ of $C_{n, m}^{k}$ has outer normal $v_{i, k}^{+}=+R e_{i}$ while the opposite face $\phi_{n, m}^{k, i-}$ has outer normal $v_{i, k}^{-}=-R e_{i}$.

We suppose now that the face $\phi_{n, m}^{k, i+}$ of $C_{n, m}^{k} \in \mathscr{C}_{n, m}$ satisfies

$$
\phi_{n, m}^{k, i+} \subset\left(1-\frac{2}{n+2}\right) Q \text {. }
$$


Then there is a cube $C_{n, m}^{k^{\prime}} \in \mathscr{C}_{n, m}$ that shares the given face with $C_{n, m}^{k}$, and we have at each point $x \in \phi_{n, m}^{k, i+}$ the equality

$$
\begin{aligned}
{\left[u_{m}^{(n)}\right](x) \cdot v(x) } & =\left(M\left(x-c_{n, m}^{k^{\prime}}\right)-M\left(x-c_{n, m}^{k}\right)\right) \cdot v_{i, k}^{+}(x) \\
& =M\left(c_{n, m}^{k}-c_{n, m}^{k^{\prime}}\right) \cdot v_{i, k}^{+}(x) \\
& =M\left(-\frac{1}{m} v_{i, k}^{+}(x)\right) \cdot v_{i, k}^{+}(x)=-\frac{1}{m} \widehat{M} \operatorname{Re}_{i} \cdot R e_{i}
\end{aligned}
$$

so that

$$
\begin{aligned}
\int_{\phi_{n, m}^{k, i+}}\left|\left[u_{m}^{(n)}\right](x) \cdot v(x)\right| d \mathcal{H}^{N-1}(x) & =\int_{\phi_{n, m}^{k, i+}} \frac{1}{m}\left|\widehat{M} R e_{i} \cdot \operatorname{Re}_{i}\right| d \mathcal{H}^{N-1}(x) \\
& =\frac{1}{m^{N}}\left|\widehat{M} R e_{i} \cdot R e_{i}\right| .
\end{aligned}
$$

The same argument shows that if

$$
\phi_{n, m}^{k, i-} \subset\left(1-\frac{2}{n+2}\right) Q
$$

then

$$
\int_{\phi_{n, m}^{k, i-}}\left|\left[u_{m}^{(n)}\right](x) \cdot v(x)\right| d \mathscr{H}^{N-1}(x)=\frac{1}{m^{N}}\left|\widehat{M} R e_{i} \cdot R e_{i}\right| .
$$

If (2-5) holds for $i=1, \ldots, N$, then we may sum the last relation over $i$ to conclude that

$$
\begin{aligned}
\sum_{i=1}^{N} \int_{\phi_{n, m}^{k, i}}\left|\left[u_{m}^{(n)}\right](x) \cdot v(x)\right| d \mathscr{H}^{N-1}(x) & =\frac{1}{m^{N}} \sum_{i=1}^{N}\left|\widehat{M} R e_{i} \cdot R e_{i}\right| \\
& \geq \frac{1}{m^{N}}\left|\sum_{i=1}^{N} \widehat{M} R e_{i} \cdot R e_{i}\right| \\
& =\frac{1}{m^{N}}\left|\sum_{i=1}^{N} R^{T} \widehat{M} R e_{i} \cdot e_{i}\right| \\
& =\frac{1}{m^{N}}\left|\operatorname{tr}\left(R^{T} \widehat{M} R\right)\right|=\frac{1}{m^{N}}|\operatorname{tr} M| .
\end{aligned}
$$

In (2-8), equality holds if and only if all of the numbers $\widehat{M} R e_{i} \cdot R e_{i}, i=1, \ldots, N$, have the same sign:

$$
\left(\widehat{M} R e_{i} \cdot R e_{i}\right)\left(\widehat{M} R e_{j} \cdot R e_{j}\right) \geq 0 \text { for } i, j=1, \ldots, N .
$$

The last two inequalities lead us to consider the problem

$$
\text { find } \min _{R R^{T}=I} \sum_{i=1}^{N}\left|\widehat{M} R e_{i} \cdot R e_{i}\right| \geq|\operatorname{tr} \widehat{M}|=|\operatorname{tr} M|,
$$

with equality holding if and only if there is an orthogonal matrix $R$ satisfying (2-9). 


\section{Aside on isotropic vectors}

We note that the sign inequality (2-9) suggests looking for unit vectors $v$ such that

$$
\widehat{M} v \cdot v=0,
$$

the isotropic vectors for $\widehat{M}$ [Ciblak and Lipkin 1998]. In particular, in the special case $\operatorname{tr} \widehat{M}=0$, the existence of $N$ mutually orthogonal isotropic vectors $v_{1}, \ldots, v_{N}$ would ensure that the matrix $R$ defined by $R e_{i}=v_{i}$ for $i=1, \ldots, N$ would satisfy (2-10) in the form $0=0$. More generally, even when $\operatorname{tr} \widehat{M} \neq 0$, the existence of isotropic vectors is useful. In fact, the symmetric matrix $\widehat{M}-\frac{1}{N}(\operatorname{tr} \widehat{M}) I$ has zero trace, so we suppose that there exist $N$ mutually orthogonal isotropic unit vectors $v_{1}, \ldots, v_{N}$ for $\widehat{M}-\frac{1}{N}(\operatorname{tr} \widehat{M}) I$. The relation (3-1) with $\widehat{M}$ replaced by $\widehat{M}-\frac{1}{N}(\operatorname{tr} \widehat{M}) I$ then becomes

$$
0=\left(\widehat{M}-\frac{1}{N}(\operatorname{tr} \widehat{M}) I\right) v_{i} \cdot v_{i}=\widehat{M} v_{i} \cdot v_{i}-\frac{1}{N} \operatorname{tr} \widehat{M}
$$

so that $\widehat{M} v_{i} \cdot v_{i}=\frac{1}{N} \operatorname{tr} \widehat{M}$ for $i=1, \ldots, N$. Again, if we define a linear mapping $R$ on $\mathbb{R}^{N}$ by $R e_{i}=v_{i}$ for $i=1, \ldots, N$, then $R$ is orthogonal, it satisfies the sign inequality for $\widehat{M}$ (2-9), and it delivers equality in (2-10) in the form $\sum_{i=1}^{N}\left|\frac{1}{N} \operatorname{tr} \widehat{M}\right|=|\operatorname{tr} \widehat{M}|$.

The following result [Ciblak and Lipkin 1998, Corollary 15] provides the desired existence of complete orthonormal sets of isotropic vectors.

Theorem 3.1. A symmetric matrix $A \in \mathbb{R}^{N \times N}$ possesses an orthonormal set of $N$ isotropic vectors if and only if $\operatorname{tr} A=0$.

This theorem and the preceding discussion permit us to conclude: for every matrix $M \in \mathbb{R}^{N \times N}$,

$$
\begin{aligned}
\min _{R R^{T}=I} \sum_{i=1}^{N}\left|M R e_{i} \cdot R e_{i}\right| & =\min _{R R^{T}=I} \sum_{i=1}^{N}\left|\widehat{M} R e_{i} \cdot R e_{i}\right| \\
& =|\operatorname{tr} \widehat{M}|=|\operatorname{tr} M|,
\end{aligned}
$$

and a minimizing rotation matrix $R$ is one carrying the orthonormal basis of $\mathbb{R}^{N}$ consisting of eigenvectors of $\widehat{M}$ into an orthonormal basis of $\mathbb{R}^{N}$ consisting of isotropic vectors of $\widehat{M}-\frac{1}{N}(\operatorname{tr} \widehat{M}) I$. For this minimizing rotation matrix, we have

$$
\left|\widehat{M} R e_{i} \cdot R e_{i}\right|=\frac{1}{N}|\operatorname{tr} M| \quad \text { for } i=1, \ldots, N .
$$

We remark that minimizers are not unique, in general, even when one eliminates trivial permutations of isotropic vectors. In fact, for $N=3$, there are examples of minimizers for which two of the three terms in $\sum_{i=1}^{3}\left|\widehat{M} R e_{i} \cdot R e_{i}\right|$ vanish, while the third equals $|\operatorname{tr} M|$, so that only two of the three vectors $R e_{i}$ are isotropic vectors for $\widehat{M}$. 
For the convenience of the reader, we provide the recursive step used in proving the existence of orthonormal bases made up of isotropic vectors for a traceless symmetric matrix $A \in \mathbb{R}^{N \times N}$. We interpret $A$ in the usual way as a linear mapping on $\mathbb{R}^{N}$, endowed with the standard inner product. Then the nullspace $\operatorname{Ker} A$ of $A$ and its orthogonal complement $(\operatorname{Ker} A)^{\perp}$ are complementary $A$-invariant subspaces of $\mathbb{R}^{N}$, and all vectors in $\operatorname{Ker} A$ are isotropic vectors for $A$. If $(\operatorname{Ker} A)^{\perp}$ is the zero subspace, then $A=0$ and every vector in $\mathbb{R}^{N}$ is an isotropic vector for $A$, and every orthonormal basis of $\mathbb{R}^{N}$ meets the desired requirement. If $(\operatorname{Ker} A)^{\perp}$ is not the zero subspace, then we seek additional isotropic vectors for $A$ in $(\operatorname{Ker} A)^{\perp}$. To this end, the traceless symmetric linear mapping $A \neq 0$ has both positive and negative eigenvalues so that

$$
\min _{|u|=1} A u \cdot u<0<\max _{|u|=1} A u \cdot u
$$

and since the unit sphere in $\mathbb{R}^{N}$ is connected and since the quadratic form $u \mapsto A u \cdot u$ is continuous, there exists a unit vector $v_{1} \in \mathbb{R}^{N}$ such that $A v_{1} \cdot v_{1}=0$. Writing $v_{1}$ as a sum of two orthogonal vectors, one in $\operatorname{Ker} A$ and the other in $(\operatorname{Ker} A)^{\perp}$, and using the invariance of $(\operatorname{Ker} A)^{\perp}$ under $A$ shows that we may without loss of generality assume that $v_{1} \in(\operatorname{Ker} A)^{\perp}$. The linear $\operatorname{span} \operatorname{Lsp}\left(\operatorname{Ker} A \cup\left\{v_{1}\right\}\right)$ has dimension one larger than that of $\operatorname{Ker} A$ and consists solely of isotropic vectors for $A$. Consequently, we need to search for isotropic vectors of $A$ in $\left(\operatorname{Lsp}\left(\operatorname{Ker} A \cup\left\{v_{1}\right\}\right)\right)^{\perp}$ which has dimension one less than $(\operatorname{Ker} A)^{\perp}$. To proceed further, we define a linear mapping $A_{1}$ on $\mathbb{R}^{N}$ by

$$
A_{1}=A-v_{1} \otimes A v_{1}-A v_{1} \otimes v_{1}
$$

where the formula $(a \otimes b) v:=(b \cdot v) a$, for all $a, b, v \in \mathbb{R}^{N}$, defines the standard tensor product $a \otimes b \in \operatorname{Lin}\left(\mathbb{R}^{N} ; \mathbb{R}^{N}\right)$. From the fact that $v_{1}$ is an isotropic vector for $A$ and from the formula $\operatorname{tr}(a \otimes b)=a \cdot b$, it is easy to see that $A_{1}$ is traceless; because $(a \otimes b)^{T}=b \otimes a$, it follows that $A_{1}$ is symmetric. In addition, if $v \in$ $\left(\operatorname{Lsp}\left(\operatorname{Ker} A \cup\left\{v_{1}\right\}\right)\right)^{\perp}$ is an isotropic vector for $A_{1}$, then we not only have $v \cdot v_{1}=0$ but also

$$
\begin{aligned}
0 & =A_{1} v \cdot v \\
& =\left(A v-\left(A v_{1} \cdot v\right) v_{1}-\left(v_{1} \cdot v\right) A v_{1}\right) \cdot v \\
& =A v \cdot v-\left(A v_{1} \cdot v\right)\left(v_{1} \cdot v\right)-\left(v_{1} \cdot v\right)\left(A v_{1} \cdot v\right) \\
& =A v \cdot v
\end{aligned}
$$

Thus, every isotropic vector for $A_{1}$ that is in $\left(\operatorname{Lsp}\left(\operatorname{Ker} A \cup\left\{v_{1}\right\}\right)\right)^{\perp}$ is an isotropic vector for $A$, and $\operatorname{dim}\left(\left(\operatorname{Lsp}\left(\operatorname{Ker} A \cup\left\{v_{1}\right\}\right)\right)^{\perp}\right)=\operatorname{dim}\left((\operatorname{Ker} A)^{\perp}\right)-1$. To be able to apply the foregoing considerations to $A_{1}$, we need only show that $\left(\operatorname{Lsp}\left(\operatorname{Ker} A \cup\left\{v_{1}\right\}\right)\right)^{\perp}$ is invariant under $A_{1}$. To this end, let $v \in\left(\operatorname{Lsp}\left(\operatorname{Ker} A \cup\left\{v_{1}\right\}\right)\right)^{\perp}, v_{\kappa} \in \operatorname{Ker} A$, and 
$\alpha \in \mathbb{R}$ be given, and consider

$$
\begin{aligned}
A_{1} v \cdot\left(v_{\kappa}+\alpha v_{1}\right) & =A_{1} v \cdot v_{\kappa}+A_{1} v \cdot \alpha v_{1} \\
& =v \cdot A_{1} v_{\kappa}+\alpha v \cdot A_{1} v_{1} \\
& =0+\alpha v \cdot\left(A v_{1}-\left(A v_{1} \otimes v_{1}\right) v_{1}-\left(v_{1} \otimes A v_{1}\right) v_{1}\right) \\
& =\alpha v \cdot\left(A v_{1}-\left(v_{1} \cdot v_{1}\right) A v_{1}-\left(A v_{1} \cdot v_{1}\right) v_{1}\right) \\
& =\alpha v \cdot\left(A v_{1}-A v_{1}-0\right)=0 .
\end{aligned}
$$

We may conclude that $A_{1} v \in\left(\operatorname{Lsp}\left(\operatorname{Ker} A \cup\left\{v_{1}\right\}\right)\right)^{\perp}$ as desired. In the third line of the above computation, we have used the side calculation

$$
\begin{aligned}
v \cdot A_{1} v_{\kappa} & =v \cdot\left(A-v_{1} \otimes A v_{1}-A v_{1} \otimes v_{1}\right) v_{\kappa} \\
& =v \cdot A v_{\kappa}-\left(A v_{1} \cdot v_{\kappa}\right)\left(v \cdot v_{1}\right)-\left(v_{1} \cdot v_{\kappa}\right)\left(v \cdot A v_{1}\right)=0
\end{aligned}
$$

The first term on the last line vanishes because $v_{\kappa} \in \operatorname{Ker} A$, the second vanishes because $v \in\left(\operatorname{Lsp}\left(\operatorname{Ker} A \cup\left\{v_{1}\right\}\right)\right)^{\perp}$, and the third vanishes because $v_{1} \in(\operatorname{Ker} A)^{\perp}$. The search for isotropic vectors for $A$ on the $A$-invariant subspace $(\operatorname{Ker} A)^{\perp}$ may now be replaced by the search for isotropic vectors for $A_{1}$ on the $A_{1}$-invariant subspace $\left(\operatorname{Lsp}\left(\operatorname{Ker} A \cup\left\{v_{1}\right\}\right)\right)^{\perp}$ of dimension one less than that of $(\operatorname{Ker} A)^{\perp}$.

\section{Completion of the proof of the upper-bound inequality}

We may use (3-2) and the formulas (2-6) and (2-7) to conclude: if $C_{n, m}^{k}$ has a face $\phi_{n, m}^{k, i \pm} \subset\left(1-\frac{2}{N+2}\right) Q$, then

$$
\int_{\phi_{n, m}^{k, i \pm}}\left|\left[u_{m}^{(n)}\right](x) \cdot v(x)\right| d \mathscr{H}^{N-1}(x)=\frac{|\operatorname{tr} M|}{N m^{N}}=\frac{|\operatorname{tr} M|}{N} \mathscr{L}^{N}\left(C_{n, m}^{k}\right) .
$$

On the other hand, if a face $\phi_{n, m}^{k, i \pm}$ of $C_{n, m}^{k} \in \mathscr{C}_{n, m}$ fails to satisfy $\phi_{n, m}^{k, i \pm} \subset\left(1-\frac{2}{N+2}\right) Q$, then the argument used to verify (4-1) may be applied to $\phi_{n, m}^{k, i \pm} \cap\left(1-\frac{2}{n+2}\right) Q$ to conclude that

$$
\int_{\phi_{n, m}^{k, i \pm} \cap(1-2 /(n+2)) Q}\left|\left[u_{m}^{(n)}\right](x) \cdot v(x)\right| d \mathscr{H}^{N-1}(x) \leq \frac{|\operatorname{tr} M|}{N} \mathscr{L}^{N}\left(C_{n, m}^{k}\right) .
$$

We now consider the cube $C_{n, m}^{1} \in \mathscr{C}_{n, m}$ and choose $V_{n, m}^{1}$, one of its $2^{N}$ vertices. Exactly $N$ faces $\phi^{1, j}, j=1, \ldots, N$, of $C_{n, m}^{1}$ meet at $V_{n, m}^{1}$. Because each cube $C_{n, m}^{k} \in \mathscr{C}_{n, m}$ for $k=1, \ldots, K_{n, m}$ can be obtained from $C_{n, m}^{1}$ by a unique translation $T_{k}$, the choices $C_{n, m}^{1}$ and $V_{n, m}^{1}$ induce via $T_{k}$ an assignment of $N$ faces $\phi^{k, j}$, $j=1, \ldots, N$, to $C_{n, m}^{k}$. It is easy to show that for all $k, k^{\prime}=1, \ldots, K_{n, m}$

$$
k^{\prime} \neq k \Longrightarrow\left\{\phi^{k^{\prime}, j}: j=1, \ldots, N\right\} \cap\left\{\phi^{k, j}: j=1, \ldots, N\right\}=\varnothing,
$$


i.e., the set of $N$ faces assigned to different cubes are disjoint. If we now apply the mapping

$$
C_{n, m}^{k} \mapsto\left\{\phi^{k, j}: j=1, \ldots, N\right\}
$$

to each cube in the collection

$$
\mathscr{C}_{n, m}^{\mathrm{int}}:=\left\{C_{n, m}^{k} \in \mathscr{C}_{n, m}: C_{n, m}^{k} \subset\left(1-\frac{2}{N+2}\right) Q\right\}
$$

then all of the faces $\phi^{k, j}$ so obtained will be included in $\left(1-\frac{2}{N+2}\right) Q$, and we may apply (4-1) to each such face to obtain for each $C_{n, m}^{k} \in \mathscr{C}_{n, m}^{\text {int }}$

$$
\sum_{j=1}^{N} \int_{\phi^{k, j}}\left|\left[u_{m}^{(n)}\right](x) \cdot v(x)\right| d \mathscr{H}^{N-1}(x)=N \frac{|\operatorname{tr} M|}{N} \mathscr{L}^{N}\left(C_{n, m}^{k}\right)=|\operatorname{tr} M| \mathscr{L}^{N}\left(C_{n, m}^{k}\right) .
$$

We may sum both sides over the cubes $C_{n, m}^{k} \in \mathscr{C}_{n, m}^{\text {int }}$ to obtain

$$
\sum_{C_{n, m}^{k} \in \mathscr{C}_{n, m}^{\text {int }}} \sum_{j=1}^{N} \int_{\phi^{k, j}}\left|\left[u_{m}^{(n)}\right](x) \cdot v(x)\right| d \mathscr{H}^{N-1}(x)=|\operatorname{tr} M| \mathscr{L}^{N}\left(\bigcup_{C_{n, m}^{k} \in \mathscr{C}_{n, m}^{\text {int }}} C_{n, m}^{k}\right) .
$$

The faces represented on the left-hand side need not include all of $J\left(u_{n, m}\right) \subset$ $\bigcup_{k=1}^{K_{n, m}} \partial C_{n, m}^{k}$, because some faces of cubes $C_{n, m}^{k} \in \mathscr{C}_{n, m}^{\text {int }}$ that are also faces of cubes $C_{n, m}^{k^{\prime}} \in \mathscr{C}_{n, m} \backslash \mathscr{C}_{n, m}^{\text {int }}$ are left out, while proper subsets $\phi_{n, m}^{k, i \pm} \cap\left(1-\frac{2}{n+2}\right) Q$ of faces $\phi_{n, m}^{k, i \pm}$ also are left out. However, for those parts of $J\left(u_{n, m}\right)$, we may use (4-1) and

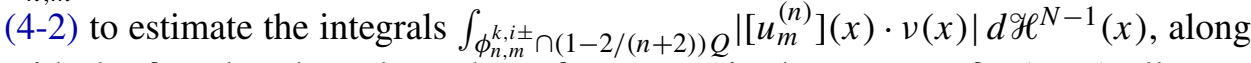
with the fact that the cubes whose faces contain these parts of $J\left(u_{n, m}\right)$ all must contain points of $\partial\left(1-\frac{2}{n+2}\right) Q$ and must together cover $\partial\left(1-\frac{2}{n+2}\right) Q$. Combining all of these contributions to $\int_{J\left(u_{n, m}\right)}\left|\left[u_{m}^{(n)}\right](x) \cdot v(x)\right| d \mathscr{H}^{N-1}(x)$, we obtain

$$
\begin{aligned}
0 & \leq \int_{J\left(u_{n, m}\right)}\left|\left[u_{m}^{(n)}\right](x) \cdot v(x)\right| d \mathscr{H}^{N-1}(x)-|\operatorname{tr} M| \mathscr{L}^{N}\left(\bigcup_{C_{n, m}^{k} \in \mathscr{C}_{n, m}^{\mathrm{int}}} C_{n, m}^{k}\right) \\
& \leq 2|\operatorname{tr} M| \mathscr{L}^{N}\left(\bigcup_{C_{n, m}^{k} \in\left(\mathscr{C}_{n, m} \backslash \mathscr{G}_{n, m}^{\mathrm{int}}\right)} C_{n, m}^{k}\right) .
\end{aligned}
$$

The factor of $2=2 N / N$ in the last expression reflects the fact that the $\mathscr{L}^{N}$-measure of some of the cubes in the collection $\mathscr{C}_{n, m} \backslash \mathscr{C}_{n, m}^{\text {int }}$ has been counted more than once but no more than $2 N$ times through the use of the bound (4-2). The relations (4-3), 
(2-2), (2-3), and (2-4) now yield the relation

$$
\begin{array}{r}
0 \leq \int_{J\left(u_{m}^{(n)}\right)}\left|\left[u_{m}^{(n)}\right](x) \cdot v(x)\right| d \mathscr{H}^{N-1}(x)-|\operatorname{tr} M| \mathscr{L}^{N}\left(\bigcup_{C_{n, m}^{k} \in \mathscr{C}_{n, m}^{\text {int }}} C_{n, m}^{k}\right) \\
\leq 2|\operatorname{tr} M| \mathscr{L}^{N}\left(\bigcup_{C_{n, m}^{k} \in\left(\mathscr{C}_{n, m} \backslash \mathscr{C}_{n, m}^{\text {int }}\right)} C_{n, m}^{k}\right)+\frac{\sqrt{N}}{m}\|M\| 2 N\left(1-\frac{2}{n+2}\right)^{N-1} \\
+C(N)\|M\|\left(1-\left(1-\frac{2}{n+2}\right)^{N}\right) .
\end{array}
$$

We in turn use (4-4) to obtain an upper bound for

$$
\int_{J\left(u_{m}^{(n)}\right)}\left|\left[u_{m}^{(n)}\right](x) \cdot v(x)\right| d \mathscr{H}^{N-1}(x) .
$$

Let $\varepsilon>0$ be given, choose $n$ so large that $C(N)\|M\|\left(1-\left(1-\frac{2}{n+2}\right)^{N}\right)<\varepsilon$, and for such an $n$, choose $m$ so large that $(\sqrt{N} / m)\|M\| 2 N\left(1-\frac{2}{n+2}\right)^{N-1}<\varepsilon$. Because $\left(1-\frac{2}{n+2}\right) Q$ has finite $\mathscr{L}^{N}$-measure, we may choose $m$ larger if necessary so that the cover $\mathscr{C}_{n, m}$ of $\left(1-\frac{2}{n+2}\right) Q$ satisfies $\mathscr{L}^{N}\left(\bigcup_{C_{n, m}^{k} \in \mathscr{C}_{n, m}} C_{n, m}^{k}\right)<$ $\mathscr{L}^{N}\left(\left(1-\frac{2}{n+2}\right) Q\right)+\varepsilon<1+\varepsilon$. Finally, because $\partial\left(1-\frac{2}{n+2}\right) Q$ has zero $\mathscr{L}^{N}$-measure and is covered by $\mathscr{C}_{n, m} \backslash \mathscr{C}_{n, m}^{\text {int }}$, we may again choose $m$ larger, if necessary, so that $2|\operatorname{tr} M| \mathscr{L}^{N}\left(\bigcup_{C_{n, m}^{k} \in\left(\mathscr{C}_{n, m} \backslash \mathscr{G}_{n, m}^{\text {int }}\right)} C_{n, m}^{k}\right)<\varepsilon$. We conclude that for $n$ and $m$ so chosen

$$
\int_{J\left(u_{m}^{(n)}\right)}\left|\left[u_{m}^{(n)}\right](x) \cdot v(x)\right| d \mathscr{H}^{N-1}(x)<|\operatorname{tr} M|(1+\varepsilon)+3 \varepsilon=|\operatorname{tr} M|+(|\operatorname{tr} M|+3) \varepsilon
$$

and, since $\varepsilon>0$ was arbitrary, that (1-27) holds.

\section{Additional explicit formulas for disarrangement densities}

Our discussion above shows that the particular choice of interfacial measure of disarrangements

$$
\int_{J(u) \cap \Omega}|[u] \cdot v| d \mathscr{H}^{N-1}
$$

for deformations $u$ of a region $\Omega \subset \mathbb{R}^{N}$ leads in both the Choksi-Fonseca relaxation scheme [1997] and in the Baía-Matias-Santos relaxation scheme [Baía et al. 2012] to the same bulk density of disarrangements

$$
\int_{\Omega}|\operatorname{tr}(\nabla g-G)| d \mathscr{L}^{N}
$$

for structured deformations $(g, G)$ of that region. Moreover, our analysis here provides an alternative to the proof of this result given in [Owen and Paroni 2015]. In that article, it was observed that replacement of $|[u] \cdot v|$ by its positive part $([u] \cdot v)^{+}=\frac{1}{2}(|[u] \cdot v|+[u] \cdot v)$ results in the replacement of $|\operatorname{tr}(\nabla g-G)|$ by its positive part $(\operatorname{tr}(\nabla g-G))^{+}=\frac{1}{2}(|\operatorname{tr}(\nabla g-G)|+\operatorname{tr}(\nabla g-G))$ in the relaxed 
bulk disarrangement density. (An analogous result holds for the negative parts, obtained by replacing "+" by "-" in the definition of the positive parts.) As pointed out in [Owen and Paroni 2015], $(\operatorname{tr}(\nabla g-G)(x))^{+}$may now be interpreted as the minimum volume fraction at a point $x \in \Omega$ that can be swept out by submacroscopic separations associated with deformations $u_{n}$ approximating the structured deformation $(g, G)$. Moreover, $(\operatorname{tr}(\nabla g-G)(x))^{-}$is the minimum volume fraction at $x$ swept out by submacroscopic switches and interpenetrations so that $|\operatorname{tr}(\nabla g-G)(x)|=(\operatorname{tr}(\nabla g-G)(x))^{+}+(\operatorname{tr}(\nabla g-G)(x))^{-}$is the minimum volume fraction swept out by submacroscopic separations, switches, and interpenetrations.

The presence of the inner product $[u] \cdot v$ in the initial interfacial density (5-1) tells us that only normal components of jumps will contribute and that alternative initial interfacial densities are required in order to capture contributions of tangential components of jumps. In the remainder of this section, we shall provide alternative initial interfacial densities that not only capture contributions of tangential components of jumps but also lead to specific formulas for the relaxed bulk disarrangement density via the "tilted cube" construction provided in Sections 2 and 4 above.

Let $a \in \mathbb{R}^{N}$ be given, and consider the replacement for (5-1)

$$
\int_{J(u) \cap \Omega}|[u] \cdot a| d \mathscr{H}^{N-1}
$$

in which the normal component $[u] \cdot v$ of the jump in $u$ is replaced by the component $[u] \cdot a$ in the direction of $a$. To again follow the relaxation scheme in [Baía et al. 2012], we let $A, B \in \mathbb{R}^{N \times N}$ be given and require not only $u \in \operatorname{SBV}\left(Q, \mathbb{R}^{N}\right)$ but also

$$
\left.u\right|_{\partial Q}=0, \quad \nabla u=B-A, \quad \mathscr{L}^{N} \text {-a.e. in } Q .
$$

We now may use the Gauss-Green formula and (5-3) to write

$$
\begin{aligned}
\int_{J(u) \cap Q}|[u] \cdot a| d \mathscr{H}^{N-1} & =\int_{J(u) \cap Q}|([u \cdot a]) v| d \mathscr{H}^{N-1} \\
& \geq\left|\int_{J(u) \cap Q}([u \cdot a]) v d \mathscr{H}^{N-1}\right| \\
& =\left|-\int_{Q} \nabla(u \cdot a) d \mathscr{L}^{N}+\int_{\partial Q}(u \cdot a) v d \mathscr{H}^{N-1}\right| \\
& =\left|-\int_{Q}(\nabla u)^{T} a d \mathscr{L}^{N}+\int_{\partial Q}(0 \cdot a) v d \mathscr{H}^{N-1}\right| \\
& =\left|(B-A)^{T} a\right| .
\end{aligned}
$$


For the "tilted-cube" construction provided in Sections 2 and 4, we replace the matrix $M$ by $B-A$, and the relation (2-6) here has the counterpart

$$
\begin{aligned}
\int_{\phi_{n, m}^{k, i+}}\left|\left[u_{m}^{(n)}\right](x) \cdot a\right| d \mathscr{H}^{N-1}(x) & =\int_{\phi_{n, m}^{k, i+}}\left|\left(\left[u_{m}^{(n)}\right](x) \cdot a\right) v(x)\right| d \mathscr{H}^{N-1}(x) \\
& =\int_{\phi_{n, m}^{k, i+}} \frac{1}{m}\left|\left((B-A) R e_{i} \cdot a\right) R e_{i}\right| d \mathcal{H}^{N-1}(x) \\
& =\frac{1}{m^{N}}\left|\left(R e_{i} \cdot(B-A)^{T} a\right) R e_{i}\right|,
\end{aligned}
$$

and this formula leads to the counterpart of (2-8)

$$
\begin{aligned}
\sum_{i=1}^{N} \int_{\phi_{n, m}^{k, i+}}\left|\left[u_{m}^{(n)}\right](x) \cdot a\right| d \mathscr{H}^{N-1}(x) & =\frac{1}{m^{N}} \sum_{i=1}^{N}\left|\left(R e_{i} \cdot(B-A)^{T} a\right) R e_{i}\right| \\
& \geq \frac{1}{m^{N}}\left|\sum_{i=1}^{N}\left(R e_{i} \cdot(B-A)^{T} a\right) R e_{i}\right| \\
& =\frac{1}{m^{N}}\left|(B-A)^{T} a\right| .
\end{aligned}
$$

The method employed in Sections 2 and 4 (where the symbol $M$ was used in place of $B-A$ ) then requires the choice of a rotation $R$ for which equality holds in the second line of (5-4). If $(B-A)^{T} a \neq 0$, we may choose $R$ to be any rotation satisfying $R e_{1}=(B-A)^{T} a /\left|(B-A)^{T} a\right|$, and this requirement is then met because $\left(R e_{i} \cdot(B-A)^{T} a\right) R e_{i}=0$ for $i=2, \ldots, N$. If $(B-A)^{T} a=0$, then $R$ can be chosen arbitrarily; for example, $R=I$ suffices.

These observations show that the analysis in Section 4 for (5-1) may be carried out step by step for the alternative initial density (5-2), provided that everywhere in Section 4 we replace $|\operatorname{tr} M|=|\operatorname{tr}(B-A)|$ by $\left|(B-A)^{T} a\right|$, the Euclidean norm of the vector $(B-A)^{T} a$. If we now define

$$
\begin{aligned}
H(A, B, a):=\inf \left\{\int_{J(u)}|[u](x) \cdot a| d \mathcal{H}^{N-1}(x): u\right. & \in S B V\left(Q ; \mathbb{R}^{N}\right), \\
& \left.\left.u\right|_{\partial Q}=0, \nabla u=B-A \text { a.e. }\right\},
\end{aligned}
$$

then our observations amount to the formula

$$
H(A, B, a)=\left|(B-A)^{T} a\right|
$$

for the relaxed bulk energy density corresponding to the initial interfacial energy (5-2) and arising from the scheme in [Baía et al. 2012]. Moreover, an argument similar to that used in establishing (1-26) shows that the formula (5-5) also holds for the relaxed bulk disarrangement density according to [Choksi and Fonseca 1997]. In 
the context of a given structured deformation $(g, G)$ on a region $\Omega$, (5-5) implies that the particular choice of initial interfacial disarrangement

$$
\int_{J(u) \cap \Omega}|[u] \cdot a| d \mathscr{H}^{N-1}
$$

for deformations $u$ of a region $\Omega \subset \mathbb{R}^{N}$ leads in both the Choksi-Fonseca relaxation scheme [1997] and in the Baía-Matias-Santos relaxation scheme [Baía et al. 2012] to the same relaxed bulk disarrangement density

$$
\int_{\Omega}\left|(\nabla g-G)^{T} a\right| d \mathscr{L}^{N}
$$

for structured deformations $(g, G)$ of that region. The integral in (5-6) represents the most economical way of introducing jumps in the direction of $a$ while approaching in the limit the given structured deformation $(g, G)$, including both jumps normal and tangential to the discontinuity surfaces of approximating deformations $u$.

We also note the formula

$$
\max _{i=1, \ldots, N} H\left(A, B, \delta_{i}\right)=\|B-A\|_{\text {row max }}
$$

where on the left $\delta_{1}, \ldots, \delta_{N}$ denote the standard basis of $\mathbb{R}^{N}$ and on the right $\|B-A\|_{\text {row max }}$ denotes the maximum of the Euclidean norms of the rows of $B-A$. The mapping $\|\cdot\|_{\text {row max }}: \mathbb{R}^{N \times N} \rightarrow \mathbb{R}$ turns out to be a norm on $\mathbb{R}^{N \times N}$, and our interpretation of the integral in (5-6) leads us to interpret the integral

$$
\int_{\Omega}\|(\nabla g-G)(x)\|_{\text {row max }} d \mathscr{L}^{N}(x)
$$

as a bulk measure of disarrangements that takes into account at each $x \in \Omega$ the direction $\delta_{i(x)}$ that maximizes the relaxed bulk energy densities $H\left(\nabla g(x), G(x), \delta_{i}\right)$ for $i=1, \ldots, N$. The bulk disarrangement density $\max _{i=1, \ldots, N} H\left(A, B, \delta_{i}\right)=$ $\|B-A\|_{\text {row max }}$ satisfies

$$
\begin{array}{r}
\max _{i=1, \ldots, N} H\left(A, B, \delta_{i}\right) \leq \inf \left\{\max _{i=1, \ldots, N} \int_{J(u)}\left|[u](x) \cdot \delta_{i}\right| d \mathscr{H}^{N-1}(x): u \in S B V\left(Q ; \mathbb{R}^{N}\right),\right. \\
\left.\left.u\right|_{\partial Q}=0, \nabla u=B-A \text { a.e. }\right\}
\end{array}
$$

and need not be the relaxed bulk energy density corresponding to the initial interfacial energy $\max _{i=1, \ldots, N} \int_{J(u)}\left|[u](x) \cdot \delta_{i}\right| d \mathscr{H}^{N-1}(x)$.

\section{Acknowledgments}

The authors warmly thank the Center for Nonlinear Analysis (NSF grants numbers DMS-0405343 and DMS-0635983) at Carnegie Mellon University and the 
Centre for Mathematical Analysis, Geometry, and Dynamical Systems (FCT grant UID/MAT/04459/2013) at Instituto Superior Técnico, where this research was carried out. The research of Barroso, Matias, and Morandotti was partially supported by the Fundação para a Ciência e a Tecnologia (Portuguese Foundation for Science and Technology) through the CMU-Portugal Program under grant FCTUTA_CMU/MAT/0005/2009 “Thin Structures, Homogenization, and Multiphase Problems". The research of Barroso was partially supported by the Fundação para a Ciência e a Tecnologia through grant PEst_OE/MAT/UI0209/2013. The research of Morandotti was partially supported by the European Research Council through the ERC Advanced Grant "QuaDynEvoPro", grant agreement 290888. Morandotti is a member of the Progetto di Ricerca GNAMPA-INdAM 2015 "Fenomeni critici nella meccanica dei materiali: un approccio variazionale" (INdAM-GNAMPA Project 2015 "Critical phenomena in the mechanics of materials: a variational approach").

\section{References}

[Baía et al. 2011] M. Baía, J. Matias, and P. M. Santos, "A survey on structured deformations", São Paulo J. Math. Sci. 5:2 (2011), 185-201.

[Baía et al. 2012] M. Baía, J. Matias, and P. M. Santos, "A relaxation result in the framework of structured deformations in a bounded variation setting", Proc. Roy. Soc. Edinburgh Sect. A 142:2 (2012), 239-271.

[Choksi and Fonseca 1997] R. Choksi and I. Fonseca, "Bulk and interfacial energy densities for structured deformations of continua", Arch. Rational Mech. Anal. 138:1 (1997), 37-103.

[Choksi et al. 1999] R. Choksi, G. Del Piero, I. Fonseca, and D. Owen, "Structured deformations as energy minimizers in models of fracture and hysteresis", Math. Mech. Solids 4:3 (1999), 321-356.

[Ciblak and Lipkin 1998] N. Ciblak and H. Lipkin, "Orthonormal isotropic vector bases", in Proceedings of DETC'98: 1998 ASME Design Engineering Technical Conferences (Atlanta, 1998), ASME International, New York, 1998.

[Del Piero and Owen 1993] G. Del Piero and D. R. Owen, "Structured deformations of continua", Arch. Rational Mech. Anal. 124:2 (1993), 99-155.

[Del Piero and Owen 1995] G. Del Piero and D. R. Owen, "Integral-gradient formulae for structured deformations", Arch. Rational Mech. Anal. 131:2 (1995), 121-138.

[Deseri and Owen 2003] L. Deseri and D. R. Owen, "Toward a field theory for elastic bodies undergoing disarrangements", J. Elasticity 70:1-3 (2003), 197-236.

[Kröner 1958] E. Kröner, Kontinuumstheorie der Versetzungen und Eigenspannungen, Ergebnisse angewandten Math. 5, Springer, Berlin, 1958.

[Matias 2007] J. Matias, "Differential inclusions in $\operatorname{SBV}_{0}(\Omega)$ and applications to the calculus of variations", J. Convex Anal. 14:3 (2007), 465-477.

[Matias and Santos 2014] J. Matias and P. M. Santos, "A dimension reduction result in the framework of structured deformations", Appl. Math. Optim. 69:3 (2014), 459-485.

[Nye 1953] J. F. Nye, "Some geometrical relations in dislocated crystals", Acta Metall. 1:2 (1953), $153-162$. 
[Owen and Paroni 2000] D. R. Owen and R. Paroni, "Second-order structured deformations", Arch. Ration. Mech. Anal. 155:3 (2000), 215-235.

[Owen and Paroni 2015] D. R. Owen and R. Paroni, "Optimal flux densities for linear mappings and the multiscale geometry of structured deformations", Arch. Ration. Mech. Anal. 218:3 (2015), $1633-1652$.

[Šilhavý 2015] M. Šilhavý, "On the approximation theorem for structured deformations from $B V(\Omega)$ ", Math. Mech. Complex Syst. 3:1 (2015), 83-100.

[Šilhavý 2016] M. Šilhavý, "Relaxed disarrangements densities for structured deformations”, preprint IM-2016-53, Institute of Mathematics, CAS, 2016, Available at http://tinyurl.com/silhavy.

Received 8 Jul 2016. Accepted 31 Oct 2016.

ANA CRISTINA BARROSO: acbarroso@ciencias.ulisboa.pt

Departamento de Matemática and Centro de Matemática, Aplicações Fundamentais e Investigação Operacional, Faculdade de Ciências da Universidade de Lisboa, Campo Grande, Edifício C6, Piso 1, 1749-016 Lisboa, Portugal

José Matias: jose.c.matias@tecnico.ulisboa.pt

Departamento de Matemática, Instituto Superior Técnico, Avenida Rovisco Pais 1, 1049-001 Lisboa, Portugal

MARCO MORANDOTTI: marco.morandotti@ma.tum.de

Fakultät für Mathematik, Technische Universität München, Boltzmannstraße 3, 85748 Garching bei München, Germany

DAVID R. OWEN: do04@andrew. cmu .edu

Department of Mathematical Sciences, Carnegie Mellon University, 5000 Forbes Avenue, Pittsburgh, PA 15213, United States 



\title{
THE GENERAL FORM OF THE RELAXATION OF A PURELY INTERFACIAL ENERGY FOR STRUCTURED DEFORMATIONS
}

\author{
MiroslaV ŠILhaVÝ
}

\begin{abstract}
This paper deals with the relaxation of energies of media with structured deformations introduced by Del Piero and Owen (1993; 1995). Structured deformations provide a multiscale geometry that captures the contributions at the macrolevel of both smooth and nonsmooth geometrical changes (disarrangements) at submacroscopic levels. The paper examines the special case of Choksi and Fonseca's (1997) energetics of structured deformations in which the unrelaxed energy does not contain the bulk contribution. Thus, the energy is purely interfacial but of a general form. New formulas for the relaxed bulk and interfacial energies are proved. The bulk relaxed energy is shown to coincide with the subadditive envelope of the unrelaxed interfacial energy while the relaxed interfacial energy is the restriction of the envelope to rank-1 tensors. Moreover, it is shown that the minimizing sequence required to define the bulk energy in the relaxation scheme of Choksi and Fonseca (1997) can be realized in the more restrictive class required in the relaxation scheme of Baía, Matias and Santos (2012), thus establishing the equality of relaxed energies of the two approaches for general purely interfacial energies. The relaxations of the specific interfacial energies of Owen and Paroni (2015) and Barroso, Matias, Morandotti and Owen (2017) are simple consequences of our general results.
\end{abstract}

\section{Introduction}

This paper deals with the relaxation of nonclassical continua modeled as media with structured deformations introduced by Del Piero and Owen [1993; 1995]. ${ }^{1}$ In their original setting, a structured deformation is a triplet $(\mathcal{K}, g, G)$ of objects of the following nature. The set $\mathcal{K} \subset \boldsymbol{R}^{3}$, the crack site, is a subset of vanishing Lebesgue measure of the reference region $\Omega$, the map $g: \Omega \backslash \mathcal{K} \rightarrow \boldsymbol{R}^{3}$, the deformation map, is piecewise continuously differentiable and injective, and $G$ is a piecewise

\section{Communicated by Gianpietro Del Piero.}

MSC2010: primary 49J45; secondary 74A60, 74G65, 15A99.

Keywords: structured deformations, relaxation, subadditive envelope, interfacial energy, bulk energy, functions of measures.

${ }^{1}$ The reader is referred to the proceedings [Del Piero and Owen 2004] and to the recent survey [Baía et al. 2011] for additional references and for further developments. 
continuous map from $\Omega \backslash \mathcal{K}$ to the set of invertible second-order tensors describing deformation without disarrangements.

Within this context, simple deformations are triples $(\mathcal{K}, g, \nabla g)$ where $g$ is a piecewise smooth injective map with jump discontinuities describing partial or full separation of pieces of the body. In view of this, in the general case of a structured deformation $(\mathcal{K}, g, G)$, the tensor

$$
H=\nabla g-G,
$$

the deformation due to disarrangements, measures the departure of $(\mathcal{K}, g, G)$ from the simple deformation $(\mathcal{K}, g, \nabla g)$.

Choksi and Fonseca [1997] introduced into the theory of structured deformations energy considerations and the ideas of relaxation. For further studies in one- and multidimensional settings, see [Del Piero 2001; 2004]. It is well-known that the existing techniques of relaxation of the calculus of variations and continuum mechanics are unable to cope with injectivity requirements. Accordingly, Choksi and Fonseca neglect the injectivity requirement; in addition, they assume weaker regularity. In their interpretation, structured deformations are pairs $(g, G)$ where $g: \Omega \rightarrow \boldsymbol{R}^{n}$ is a special $\boldsymbol{R}^{n}$-valued map of bounded variation from the space $S B V(\Omega)$ and $G: \Omega \rightarrow$ Lin is an integrable Lin-valued map from the space $\mathrm{七}^{1}(\Omega$, Lin $) .^{2}$ Thus,

$$
S D(\Omega):=S B V(\Omega) \times \longleftarrow^{1}(\Omega, \text { Lin })
$$

is the set of all structured deformations. Structured deformations of the form $(g, \nabla g)$ with $g \in S B V(\Omega)$ are called simple deformations in this paper.

The relaxation starts from the energy

$$
E(g)=\int_{\Omega} W(\nabla g) d \mathcal{V}+\int_{J(g)} \psi\left(\llbracket g \rrbracket, v_{g}\right) d \mathcal{A}
$$

of a simple deformation $g \in S B V(\Omega)$. Here $\mathcal{V}$ and $\mathcal{A}$ are the Lebesgue measure and the $(n-1)$-dimensional Hausdorff measure in $\boldsymbol{R}^{n}$ and $\nabla g$ is the absolutely continuous part of the derivative (= gradient) $\mathrm{D} g$ of $g$, while the singular part

$$
\mathrm{D}^{\mathrm{s}} g:=\llbracket g \rrbracket \otimes v_{g} \mathcal{A}\llcorner J(g)
$$

is a tensor-valued singular measure describing the discontinuities of $g$; that part is formed from the jump set $J(g) \subset \Omega$ of $g$, the jump $\llbracket g \rrbracket$ of $g$ on $J(g)$ and the normal $v_{g}$ to $J(g)$. The reader is referred to (24), below, for a detailed description of these objects. The material is characterized by the bulk energy density $W: \operatorname{Lin} \rightarrow \boldsymbol{R}$ and

${ }^{2}$ For brevity of notation, we omit the target spaces and write $S B V(\Omega) \equiv S B V\left(\Omega, \boldsymbol{R}^{n}\right)$ and $\mathrm{七}^{1}(\Omega$, Lin $) \equiv L^{1}(\Omega$, Lin $)$. See Section 3 for more notation and detailed definitions. 
by the interfacial (or cohesion) energy $\psi: \boldsymbol{D}_{n} \rightarrow \boldsymbol{R}$, where we denote

$$
\boldsymbol{D}_{n}=\boldsymbol{R}^{n} \times \boldsymbol{S}^{n-1} .
$$

The approximation theorem of Del Piero and Owen [1993, Theorem 5.8] says that every structured deformation is a well-defined limit of simple deformations. In the framework of Choksi and Fonseca [1997] (see also [Šilhavý 2015]), this means that corresponding to each structured deformation $(g, G) \in S D(\Omega)$ there exists a sequence $\left(g_{k}, \nabla g_{k}\right) \in S D(\Omega)$ (i.e., with $g_{k}$ in $\left.S B V(\Omega)\right)$ such that

$$
\begin{gathered}
g_{k} \rightarrow g \quad \text { in } \longleftarrow^{1}\left(\Omega, \boldsymbol{R}^{n}\right), \\
\nabla g_{k} \rightarrow^{*} G \quad \text { in } \mathcal{M}(\Omega, \text { Lin }), \\
\sup \left\{\left|\nabla g_{k}\right|_{\bigsqcup^{1}\left(\Omega, \boldsymbol{R}^{n}\right)}: k=1, \ldots\right\}<\infty .
\end{gathered}
$$

The relaxed energy of a structured deformation $(g, G) \in S D(\Omega)$ is defined by

$$
I(g, G)=\inf \left\{\liminf _{k \rightarrow \infty} E\left(g_{k}\right): g_{k} \in S B V(\Omega) \text { satisfies }(2)\right\} .
$$

Thus, a sequence approaching the above infimum realizes the most economical way to build up the deformation $(g, G)$ using approximations in $S B V$. The relaxation theorem of Choksi and Fonseca [1997, Theorems 2.6 and 2.17, Remark 3.3] says that, under some assumptions on $W$ and $\psi$ (a particular case of which are Assumptions 2.1, below), the relaxed energy admits the integral representation

$$
I(g, G)=\int_{\Omega} H(\nabla g, G) d \mathcal{V}+\int_{J(g)} h\left(\llbracket g \rrbracket, v_{g}\right) d \mathcal{A}
$$

where $H$ and $h$ are some functions determined explicitly in the cited theorems (Theorem 2.2 presents formulas for $H$ and $h$ for a particular case).

This paper deals with the relaxation of energy functions $E$ for which the bulk contribution vanishes, i.e., with energy functions of the form

$$
E(g)=\int_{J(g)} \psi\left(\llbracket g \rrbracket, v_{g}\right) d \mathcal{A}
$$

for each $g \in S B V(\Omega)$. The main result, Theorem 2.3, below, gives explicit descriptions of the functions $H$ and $h$ from (4) and applies them to give simplified proofs of two particular cases Examples 2.5 and 2.6 given previously in [Owen and Paroni 2015; Barroso et al. 2017].

\section{The main result and examples}

We make the following standing hypotheses about $\psi$.

2.1. Assumptions. (i) The function $\psi: \boldsymbol{D}_{n} \rightarrow \boldsymbol{R}$ is continuous. 
(ii) We have $\psi(-a,-b)=\psi(a, b)$ and

$$
0 \leq \psi(a, b) \leq C_{1}|a|
$$

for every $(a, b) \in \boldsymbol{D}_{n}$ and some $C_{1}>0$.

(iii) The function $\psi(\cdot, v)$ is subadditive and positively homogeneous for each $v \in S^{n-1}$.

To ease the statements of the results, we extend any function $\zeta: \boldsymbol{D}_{n} \rightarrow[0, \infty)$ to an identically denoted function $\zeta: \boldsymbol{R}^{n} \times \boldsymbol{R}^{n} \rightarrow[0, \infty)$ by homogeneity with respect to the second variable, i.e., by assuming that the extended function satisfies

$$
\zeta(a, t b)=t \zeta(a, b)
$$

for any $t \geq 0$ and $(a, b) \in \boldsymbol{R}^{n} \times \boldsymbol{R}^{n}$. This convention applies in particular to the functions $\psi$ and $h$.

We need some notation to formulate the main results. Let $Q=\left(-\frac{1}{2}, \frac{1}{2}\right)^{n}$, and for every $M \in \operatorname{Lin}$, let $w_{M}: \partial Q \rightarrow \boldsymbol{R}^{n}$ be given by

$$
w_{M}(x)=M x \quad \text { for every } x \in \partial Q \text {. }
$$

Furthermore, if $(a, b) \in \boldsymbol{D}_{n}$, let $Q_{b}$ be any cube with unit edge, center at $0 \in \boldsymbol{R}^{n}$ and two faces normal to $b$, and let $z_{a, b}: Q_{b} \rightarrow \boldsymbol{R}^{n}$ be the map defined by

$$
z_{a, b}(x)=\frac{1}{2} a(\operatorname{sgn}(x \cdot b)+1), \quad x \in Q_{b} .
$$

Finally, if $u \in S B V(\Omega)$, let us put

$$
\Psi\left(\mathrm{D}^{\mathrm{s}} u\right):=\int_{J(u)} \psi\left(\llbracket u \rrbracket, v_{u}\right) d \mathcal{A} .
$$

The following statement is a particular case $W=0$ of the relaxation theorem of Choksi and Fonseca [1997, Theorems 2.6 and 2.17, Remark 3.3].

2.2. Theorem. The effective energies $H$ and $h$ are given by

$$
H(A, B)=\inf \left\{\Psi\left(\mathrm{D}^{\mathrm{s}} u\right): u \in S B V(Q), u=w_{A} \text { on } \partial Q, \int_{Q} \nabla u d \mathcal{V}=B\right\}
$$

for each $A, B \in$ Lin and

$$
h(a, b)=\inf \left\{\Psi\left(\mathrm{D}^{\mathrm{s}} u\right): u \in \operatorname{SBV}\left(Q_{b}\right), u=z_{a, b} \text { on } \partial Q_{b}, \nabla u=0 \text { on } Q_{b}\right\}
$$

for each $(a, b) \in \boldsymbol{D}_{n}$.

The following theorem, the main result of this paper, shows that the functions $H$ and $h$ admit a much more explicit description in terms of a single function $\Phi$. 
2.3. Theorem. The functions $H$ and $h$ in Theorem 2.2 are given by

$$
\begin{gathered}
H(A, B)=\Phi(A-B), \\
h(a, b)=\Phi(a \otimes b)
\end{gathered}
$$

for every $A, B \in \operatorname{Lin}$ and $(a, b) \in \boldsymbol{D}_{n}$, where $\Phi$ is a subadditive and positively homogeneous function on Lin defined by each of the following equivalent Assertions (i)-(iv); moreover, for dyadic arguments, we have an additional Assertion (v).

(i) $\Phi$ is the biggest subadditive function on Lin satisfying

$$
\Phi(a \otimes b) \leq \psi(a, b) \quad \text { for every }(a, b) \in \boldsymbol{D}_{n} ;
$$

i.e.,

$\Phi(M)=\sup \{\Theta(M): \Theta$ is subadditive on Lin

$$
\text { and } \left.\Theta(a \otimes b) \leq \psi(a, b) \text { for every }(a, b) \in \boldsymbol{D}_{n}\right\} \text {. }
$$

(ii) For every $M \in$ Lin, $^{3}$

$$
\Phi(M)=\inf \left\{\sum_{i=1}^{m} \psi\left(a_{i}, b_{i}\right):\left(a_{i}, b_{i}\right) \in \boldsymbol{D}_{n}, i=1, \ldots, m, \sum_{i=1}^{m} a_{i} \otimes b_{i}=M\right\} .
$$

(iii) For every $M \in$ Lin,

$$
\Phi(M)=\inf \left\{\Psi\left(\mathrm{D}^{\mathrm{s}} u\right): u \in S B V(Q), u=w_{M} \text { on } \partial Q, \nabla u=0 \text { on } Q\right\} .
$$

(iv) For every $M \in$ Lin,

$$
\Phi(M)=\inf \left\{\Psi\left(\mathrm{D}^{\mathrm{s}} u\right): u \in S B V(Q), u=w_{M} \text { on } \partial Q, \int_{Q} \nabla u d \mathcal{V}=0\right\} .
$$

(v) For arguments of the form $a \otimes b$, where $(a, b) \in \boldsymbol{D}_{n}$, $\Phi(a \otimes b)=\inf \left\{\Psi\left(\mathrm{D}^{\mathrm{s}} u\right): u \in \operatorname{SBV}\left(Q_{b}\right), u=z_{a, b}\right.$ on $\partial Q_{b}, \nabla u=0$ on $\left.Q_{b}\right\}$.

The proof of Theorem 2.3 is given in Sections 5 and 6, below.

2.4. Remarks. (a) Since the pointwise supremum of any family of subadditive functions is subadditive (e.g., [Hille and Phillips 1957, Theorem 7.2.2]), (15) really defines a subadditive function.

(b) Among the above characterizations of $\Phi$, the closely related novel forms (i) and (ii) must be considered as the most important. The main advantage of (i) and (ii) is that they establish connections to the wealth of results of the convexity theory. These will be employed to analyze the examples to be formulated below.

3 Throughout the paper, the letter $m$ denotes any positive integer. 
(c) In one dimension, one can orient the normals to jumps to be always the vector +1 (rather than -1 ) and hence the dependence of $\psi$ on the second variable can be suppressed: $\psi=\psi(a), a \in \boldsymbol{R}$. Assumption 2.1(iii) then says that $\psi$ is subadditive and positively homogeneous. Thus, the subadditive envelope $\Phi$ of $\psi$ is $\psi$ itself, and all mentions of a subadditive envelope can be avoided. This is not the case if Assumption 2.1(iii) is relaxed. Indeed, working in one dimension, Del Piero [2001; 2004] calculated the relaxation of the energy (1) with the interfacial energy $\psi$ of a general form, avoiding Assumption 2.1(iii). His main result contains the subadditive envelope of $\psi$ also. In light of the above discussion, this envelope plays a different but related role. The relaxation of a purely interfacial energy of a more general form than that postulated in Assumptions 2.1 in arbitrary dimension will be treated in a future paper.

(d) The expressions in (iii)-(v) already occurred previously, albeit without noting that they are mutually equivalent and equivalent to (i) and (ii), except for some particular cases to be mentioned below. The formula for $H$ in (13a) with $\Phi$ defined in (iv) and the formula for $h$ in (13b) with $\Phi$ defined in (v) are direct consequences of Choksi and Fonseca's expressions in (11) and (12). The formula for $H$ with $\Phi$ given by (iii) crops up in the relaxation schemes by Baía, Matias and Santos [Baía et al. 2012, (3.2)] and by Barroso, Matias, Morandotti and Owen [Barroso et al. 2017, Theorem 3.2]. The relaxation schemes in the last two papers require among other things higher regularity of structured deformations and are not strictly comparable with that of Choksi and Fonseca described above.

(e) The infimum (iii) could be, in principle, bigger than (iv). Nevertheless, the infima are generally the same. This has been established previously in [Barroso et al. 2017] for the special choices of $\psi$ described in the following examples, which motivated the present study.

2.5. Example [Owen and Paroni 2015, Theorem 4, particular case $L=\mathrm{I}$ ]. If

$$
\psi_{|\cdot|}(a, b)=|a \cdot b| \quad \text { and } \quad \psi_{ \pm}(a, b)=\{a \cdot b\}_{ \pm}
$$

for every $(a, b) \in \boldsymbol{D}_{n}$, where $\{\cdot\}_{+}$and $\{\cdot\}_{-}$denote the positive and negative parts of a real number, then

$$
\begin{aligned}
\Phi_{|\cdot|}(M) & =|\operatorname{tr} M|, \\
\Phi_{ \pm}(M) & =\{\operatorname{tr} M\}_{ \pm}
\end{aligned}
$$

for every $M \in$ Lin. The effective energies $H_{|\cdot|}, H_{ \pm}, h_{|\cdot|}$ and $h_{ \pm}$are determined through $\Phi_{|\cdot|}$ and $\Phi_{ \pm}$by (13).

As shown in [Owen and Paroni 2015], $\{\operatorname{tr} M\}_{+}$is a volume density of disarrangements due to submacroscopic separations, $\{\operatorname{tr} M\}_{-}$is a volume density of 
disarrangements due to submacroscopic switches and interpenetrations, and $|\operatorname{tr} M|$ is a volume density of all three of these nontangential disarrangements: separations, switches and interpenetrations. The evaluation in [Owen and Paroni 2015] of $H$ (equivalently, of $\Phi$ ) for (21) is rather complicated; a recent paper by Barroso, Matias, Morandotti and Owen [Barroso et al. 2017] presents some simplification and the realization of the minimizing sequence in the narrower class (iv) in Theorem 2.3 mentioned earlier. Our version of the derivation, which includes the minimizing sequence from (iv) via Theorem 2.3 also, is given in Section 7.

2.6. Example [Barroso et al. 2017, (5.3)]. If

$$
\psi(a, b)=|a \cdot p|
$$

for $(a, b) \in \boldsymbol{D}_{n}$, where $p \in \boldsymbol{R}^{n}$ is a fixed vector, then

$$
\Phi(M)=\left|M^{\mathrm{T}} p\right|
$$

for any $M \in \operatorname{Lin}$.

\section{Notation and functions of bounded variation}

We denote by $\boldsymbol{Z}$ the set of integers, by $\boldsymbol{N}$ the set of positive integers, by $\boldsymbol{S}^{n-1}$ the unit sphere in $\boldsymbol{R}^{n}$ and by Lin the set of all linear transformations from $\boldsymbol{R}^{n}$ into itself, often identified with the set of $n \times n$ matrices with real elements. We use the symbols "." and " $|\cdot|$ " to denote the scalar product and the euclidean norm on $\boldsymbol{R}^{n}$ and on Lin. The latter are defined by $A \cdot B:=\operatorname{tr}\left(A B^{\mathrm{T}}\right)$ and $|A|=\sqrt{A \cdot A}$ where $A^{\mathrm{T}} \in \mathrm{Lin}$ is the transpose of $A$ and tr denotes the trace.

A real-valued function $f$ defined on a vector space $\mathrm{X}$ is said to be subadditive if $f(x+y) \leq f(x)+f(y)$ for every $x, y \in \mathrm{X}$ and positively homogeneous if $f(t x)=t f(x)$ for every $t \geq 0$ and $x \in \mathrm{X}$.

If $\Omega$ is an open subset of $\boldsymbol{R}^{n}$, we denote by $\longleftarrow^{1}(\Omega$, Lin) the space of Lin-valued integrable maps on $\Omega$. We denote by $\mathcal{M}(\Omega$, Lin) the set of all (finite) Lin-valued measures on $\Omega$. If $\mu \in \mathcal{M}(\Omega, \operatorname{Lin})$, we denote by $\mu\llcorner B$ the restriction of $\mu$ to a Borel set $B \subset \Omega$. If $G, G_{k} \in \mathrm{t}^{1}(\Omega, \mathrm{Lin}), k=1,2, \ldots$, we say that $G_{k}$ converges to $G$ in the sense of measures, and write

$$
G_{k} \rightarrow^{*} G \text { in } \mathcal{M}(\Omega, \text { Lin }),
$$

if $\int_{\Omega} G_{k} \cdot H d \mathcal{V} \rightarrow \int_{\Omega} G \cdot H d \mathcal{V}$ for every continuous map $H: \boldsymbol{R}^{n} \rightarrow$ Lin which vanishes outside $\Omega$.

We state some basic definitions and properties of the space $B V(\Omega)=B V\left(\Omega, \boldsymbol{R}^{n}\right)$ of maps of bounded variation and of the space $S B V(\Omega)=S B V\left(\Omega, \boldsymbol{R}^{n}\right)$, special maps of bounded variation. For more details, see [Ambrosio et al. 2000; Evans and Gariepy 1992; Ziemer 1989; Federer 1969]. 
We define the set $B V(\Omega)$ as the set of all $u \in L^{1}(\Omega)=L^{1}\left(\Omega, \boldsymbol{R}^{n}\right)$ such that there exists a measure $\mathrm{D} u \in \mathcal{M}(\Omega$, Lin) satisfying

$$
\int_{\Omega} u \cdot \operatorname{div} T d \mathcal{V}=-\int_{\Omega} T \cdot d \mathrm{D} u
$$

for each infinitely differentiable map $T: \boldsymbol{R}^{n} \rightarrow \boldsymbol{R}^{n \times n}$ which vanishes outside some compact subset of $\Omega$. Here $\operatorname{div} T$ is an $\boldsymbol{R}^{n}$-valued map on $\Omega$ given by $(\operatorname{div} T)_{i}=$ $\sum_{j=1}^{n} T_{i j, j}$, where the comma followed by an index $j$ denotes the partial derivative with respect to $j$-th variable. The measure $\mathrm{D} u$ is uniquely determined and called the weak (or generalized) derivative of $u$. We shall need the following form of the Gauss-Green theorem for $B V$ : if $\Omega$ is a domain with lipschitzian boundary and $u \in B V(\Omega)$, then there exists an $\mathcal{A}$ integrable map $u^{\partial \Omega}: \partial \Omega \rightarrow \boldsymbol{R}^{n}$ such that

$$
\mathrm{D} u(\Omega) \equiv \int_{\Omega} d \mathrm{D} u=\int_{\partial(\Omega)} u^{\partial \Omega} \otimes v_{\Omega} d \mathcal{A},
$$

where $v_{\Omega}$ is the outer normal to $\partial \Omega$. The map $u^{\partial \Omega}$ is determined within a change on a set of $\mathcal{A}$ measure 0 and is called the trace of $u$.

We define the set $\operatorname{SBV}(\Omega)$ as the set of all $u \in B V(\Omega)$ for which $\mathrm{D} u$ has the form

$$
\mathrm{D} u=\nabla u \mathcal{V}\left\llcorner\Omega+\llbracket u \rrbracket \otimes v_{u} \mathcal{A}\llcorner J(u)\right.
$$

where $\nabla u$, the absolutely continuous part of $\mathrm{D} u$, is a map in $亡^{1}(\Omega$, Lin) and the term

$$
\mathrm{D}^{\mathrm{s}} u:=\llbracket u \rrbracket \otimes v_{u} \mathcal{A}\llcorner J(u)
$$

on the right-hand side of (24) is called the jump (or singular) part of Du. The objects $J(u) \subset \Omega, \llbracket u \rrbracket: J(u) \rightarrow \boldsymbol{R}^{n}$ and $v_{u}: J(u) \rightarrow \boldsymbol{S}^{n-1}$ are called the jump set of $u$, the jump of $u$ and the normal to $J(u)$, respectively. Here $J(u)$ is the set of all $x \in \Omega$ for which there exist $v_{u}(x) \in \boldsymbol{S}^{n-1}$ and $u^{ \pm}(x) \in \boldsymbol{R}^{n}$ such that we have the approximate limits

$$
u^{ \pm}(x)=\operatorname{ap}_{\substack{y \rightarrow x \\ y \in \boldsymbol{H}^{ \pm}\left(x, v_{u}(x)\right)}} u(x)
$$

where $\boldsymbol{H}^{ \pm}\left(x, v_{u}(x)\right)=\left\{y \in \boldsymbol{R}^{n}: \pm(y-x) \cdot v_{u}(x)>0\right\}$. For a given $x \in \Omega$, either the triplet $\left(v_{u}, u^{+}, u^{-}\right)=\left(v_{u}(x), u^{+}(x), u^{-}(x)\right)$ does not exist or it is uniquely determined to within the change $\left(v_{u}, u^{+}, u^{-}\right) \mapsto\left(-v_{u}, u^{-}, u^{+}\right)$. With one of these choices, one puts $\llbracket u \rrbracket=u^{+}-u^{-}$and notes that $\llbracket u \rrbracket \otimes v_{u}$ is unique.

Finally, we denote by $\langle r\rangle$ the integral part of $r \in \boldsymbol{R}$. Clearly,

$$
\begin{aligned}
& r-1 \leq\langle r\rangle \leq r, \\
& 0 \leq r-\langle r\rangle \leq 1 .
\end{aligned}
$$


Writing $r=k t$, where $t \in \boldsymbol{R}$ and $k>0$, and dividing by $k$, we obtain

$$
0 \leq t-\langle k t\rangle / k \leq 1 / k
$$

and hence

$$
\langle k t\rangle / k \rightarrow t \quad \text { as } k \rightarrow \infty
$$

uniformly in $t \in \boldsymbol{R}$.

\section{Preliminary results}

We put

$$
\begin{aligned}
& \mathcal{C}(M):=\left\{u \in \operatorname{SBV}(Q): u=w_{M} \text { on } \partial Q, \nabla u=0 \text { on } Q\right\}, \\
& \mathcal{B}(M):=\left\{u \in \operatorname{SBV}(Q): u=w_{M} \text { on } \partial Q, \int_{Q} \nabla u d \mathcal{V}=0\right\}
\end{aligned}
$$

for any $M \in$ Lin. We start with the following preliminary results.

4.1. Proposition. If $A, B \in \operatorname{Lin}$ and $u \in \mathcal{B}(A)$ and $v \in \mathcal{B}(B)$, then $u+v \in \mathcal{B}(A+B)$ and

$$
\Psi\left(\mathrm{D}^{\mathrm{s}} u+\mathrm{D}^{\mathrm{s}} v\right) \leq \Psi\left(\mathrm{D}^{\mathrm{s}} u\right)+\Psi\left(\mathrm{D}^{\mathrm{s}} v\right)
$$

if $(J(u) \cap J(v))=0$, then we have the equality sign in (28).

Proof. We have

$$
J(u+v)=K_{u} \cup K_{v} \cup L
$$

where

$$
L=J(u) \cap J(v), \quad K_{u}=J(u) \backslash K, \quad K_{v}=J(v) \backslash K .
$$

Next, we observe that on $L$ we have $v_{u}(x)= \pm v_{v}(x)$ for $\mathcal{A}$-almost every $x \in L$; since we have a freedom in the choice of the sign of $v_{v}$, we assume $v_{u}(x)=v_{v}(x)$ and denote $\mu=v_{u}$ on $L$. Then

$$
[u+v] \otimes v_{u+v}= \begin{cases}{[u] \otimes v_{u}} & \text { on } K_{u}, \\ {[v] \otimes v_{v}} & \text { on } K_{v}, \\ ([u]+[v]) \otimes \mu & \text { on } L .\end{cases}
$$

By the subadditivity of $\psi$,

$$
\psi([u]+[v], \mu) \leq \psi([u], \mu)+\psi([v], \mu)=\psi\left([u], v_{u}\right)+\psi\left([v], v_{v}\right)
$$

and hence (30) provides

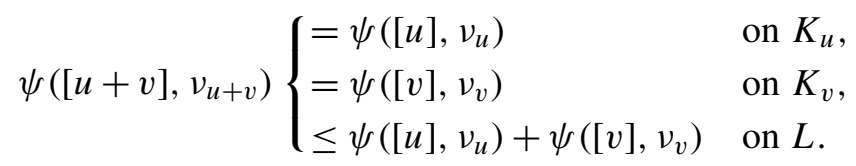


Integrating over $J(u+v)$ and using (29), we obtain

$$
\begin{aligned}
\Psi\left(\mathrm{D}^{\mathrm{s}} u+\mathrm{D}^{\mathrm{s}} v\right) & =\int_{J(u+v)} \psi\left([u+v], v_{u+v}\right) d \\
& \leq \int_{K_{u}} \psi\left([u], v_{u}\right) d+\int_{K_{v}} \psi\left([v], v_{v}\right) d \\
& \quad+\int_{L} \psi\left([u], v_{u}\right) d+\int_{L} \psi\left([v], v_{v}\right) d \\
& =\Psi\left(\mathrm{D}^{\mathrm{s}} u\right)+\Psi\left(\mathrm{D}^{\mathrm{s}} v\right),
\end{aligned}
$$

which completes the proof of (28).

4.2. Remark. If the interfacial energy density $\psi$ has the special form

$$
\psi(a, b)=\Lambda(a \otimes b)
$$

where $\Lambda: \operatorname{Lin} \rightarrow[0, \infty)$ is a subadditive and positively homogeneous function, then $\Psi\left(\mathrm{D}^{\mathrm{s}} u\right)$ is given by

$$
\Psi\left(\mathrm{D}^{\mathrm{s}} u\right)=\Lambda\left(\mathrm{D}^{\mathrm{s}} u\right)
$$

where $\mathrm{D}^{\mathrm{s}} u:=\llbracket u \rrbracket \otimes v_{u}\llcorner J(u)$ is the singular part of the derivative $\mathrm{D} u$ of $u$ and

$$
\Lambda\left(\mathrm{D}^{\mathrm{s}} u\right):=\int_{J(u)} \Lambda\left(\llbracket u \rrbracket \otimes v_{u}\right) d
$$

is an instance of Reshetnyak's [1968] functional $\mu \mapsto \Lambda(\mu)$ of a measure $\mu \in$ $\mathcal{M}(Q$, Lin $)$; see, e.g., [Ambrosio et al. 2000, (2.29)]. The subadditivity and positive homogeneity of $\Phi$ (asserted in Theorem 2.3) is then an instance of the general result [Ambrosio et al. 2000, Proposition 2.37] asserting the same properties of the functional $\mu \mapsto \Lambda(\mu)$. Indeed, if $M_{i} \in$ Lin and $u_{i} \in \mathcal{A}\left(M_{i}\right), i=1,2$, then $u_{1}+u_{2} \in \mathcal{A}\left(M_{1}+M_{2}\right)$ and therefore

$$
\Phi\left(M_{1}+M_{2}\right) \leq \Lambda\left(\mathrm{D}^{\mathrm{s}}\left(u_{1}+u_{2}\right)\right)=\Lambda\left(\mathrm{D}^{\mathrm{s}} u_{1}+\mathrm{D}^{\mathrm{s}} u_{2}\right) \leq \Lambda\left(\mathrm{D}^{\mathrm{s}} u_{1}\right)+\Lambda\left(\mathrm{D}^{\mathrm{s}} u_{2}\right)
$$

taking the infimum over all $u_{1} \in \mathcal{A}\left(M_{1}\right)$ and $u_{2} \in \mathcal{A}\left(M_{2}\right)$ gives

$$
\Phi\left(M_{1}+M_{2}\right) \leq \Phi\left(M_{1}\right)+\Phi\left(M_{2}\right) .
$$

The positive homogeneity follows similarly. We note that the interfacial energies in Examples 2.5 and 2.6 have the form (31), but this is not the case generally.

The following elementary result records some formulas to be employed below.

4.3. Remark. Let $\Omega \subset \boldsymbol{R}^{n}$ be an open bounded set with lipschitzian boundary. A countable family $\Omega_{\alpha}, \alpha \in N$, of pairwise disjoint subsets of $\Omega$ with lipschitzian boundaries is said to be a partition of $\Omega$ if one can write $\Omega=\bigcup_{\alpha=1}^{\infty} \Omega_{\alpha}$ to within a set of null Lebesgue measure. Let us agree to say that $\varphi \in L^{1}(\Omega, \boldsymbol{R})$ is piecewise constant if there exists a partition $\Omega_{\alpha}$ such that $\varphi$ is constant on each $\Omega_{\alpha}$. If $v_{\alpha}$ is 
the outer normal to $\Omega_{\alpha}$ and if $a_{\alpha}$ is the value of $\varphi$ on $\Omega_{\alpha}$, then $\varphi \in B V(\Omega, \boldsymbol{R})$ if and only if

$$
\sum_{(\alpha, \beta) \in \mathcal{I}} \int_{\partial \Omega_{\alpha} \cap \partial \Omega_{\beta}}\left|a_{\alpha}-a_{\beta}\right| d \mathcal{A}<\infty,
$$

where

$$
\mathcal{I}=\left\{(\alpha, \beta) \in N^{2}: \alpha<\beta,\left(\partial \Omega_{\alpha} \cap \partial \Omega_{\beta}\right)>0\right\} .
$$

If this is the case, we have the formulas

$$
\begin{aligned}
J(\varphi) & =\bigcup_{(\alpha, \beta) \in \mathcal{I}}\left(\partial \Omega_{\alpha} \cap \partial \Omega_{\beta}\right), \\
\llbracket \varphi \rrbracket v_{\varphi} & =\left(a_{\alpha}-a_{\beta}\right) v_{\beta} \quad \text { on } \partial \Omega_{\alpha} \cap \partial \Omega_{\beta} \text { for any }(\alpha, \beta) \in \mathcal{I}, \\
\mathrm{D} \varphi & =\llbracket \varphi \rrbracket v_{\varphi}\llcorner J(\varphi)
\end{aligned}
$$

to within changes on sets of null $\mathcal{A}$ measure. The total variation (mass) $\mathrm{M}(\mathrm{D} \varphi)$ of $\mathrm{D} \varphi$ is equal to the sum in (32).

Proof. Assume that (32) holds, and prove that $\varphi \in B V(\Omega, \boldsymbol{R})$ and that the three formulas above hold. We note that if (32) holds then $\mu:=\llbracket \varphi \rrbracket v_{\varphi}\llcorner J(\varphi)$ is a ("finite") measure in $\mathcal{M}\left(\Omega, \boldsymbol{R}^{n}\right)$. Let us prove that $\mu$ is the weak derivative of $\varphi$, which will also prove $\varphi \in B V(\Omega, \boldsymbol{R})$. Thus, we have to prove that

$$
\int_{\Omega} \varphi \nabla f d \mathcal{V}=-\int_{J(\varphi)} f \llbracket \varphi \rrbracket d \mathcal{A}
$$

for every class-infinity function $f$ with support in $\Omega$. The application of the GaussGreen theorem to each of the sets $\Omega_{\alpha}$ provides

$$
\int_{\Omega_{\alpha}} \varphi \nabla f d \mathcal{V} \equiv a_{\alpha} \int_{\Omega_{\alpha}} \nabla f d \mathcal{V}=a_{\alpha} \int_{\partial \Omega_{\alpha}} f v_{\alpha} d \mathcal{A} .
$$

Summing these equations over all $\alpha$ and using that $v_{\alpha}=-v_{\beta}$, one obtains (34) and hence we have $\varphi \in B V(\Omega, \boldsymbol{R})$, (33) and all the remaining assertions of the remark. The converse implication is proved by reversing the above arguments.

\section{The function $\Phi$}

The goal of this section is to prove that the functions defined in items (i)-(iv) of Theorem 2.3 coincide. We denote these functions by $\Phi_{1}, \Phi_{2}, \Phi_{3}$ and $\Phi_{4}$, respectively, and prove that they are the same by establishing the cycle of relations

$$
\Phi_{1} \geq \Phi_{2} \geq \Phi_{3} \geq \Phi_{4}=\Phi_{1} .
$$

5.1. Proposition. $\Phi_{1} \geq \Phi_{2}$. 
Proof. It is easy to show that $\Phi_{2}$ is a subadditive function. Thus, the definition of $\Phi_{1}$ gives the assertion.

The proof of the following lemma contains a construction of the central minimizing sequence $u_{k} \in \mathcal{C}(M)$ for Theorem 2.3(iii). This sequence will be defined as the superposition of (a slight modification of) the sequence of step deformations $s_{k}, k=1, \ldots$, defined on $Q$ by

$$
s_{k}(x)=k^{-1} a\langle k x \cdot b\rangle,
$$

$x \in Q$. Clearly, $\nabla s_{k}=0$, and in view of (27),

$$
s_{k}(x) \rightarrow a(x \cdot b) \quad \text { on } Q
$$

as $k \rightarrow \infty$. Thus, $s_{k}$ satisfies the boundary condition $s_{k}=w_{a \otimes b}$ on $\partial Q$ in the asymptotic sense; however, the definition of $\mathcal{C}(a \otimes b)$ requires the exact form of that boundary condition. For this reason, we have to slightly modify $s_{k}$ near the boundary $\partial Q$ without violating the equation $\nabla s_{k}=0$.

5.2. Lemma. If $M \in \operatorname{Lin}$ and $\left(a_{i}, b_{i}\right) \in \boldsymbol{D}_{n}, i=1, \ldots, m$, satisfy

$$
M=\sum_{i=1}^{m} a_{i} \otimes b_{i},
$$

then there exists a sequence $u_{k} \in \mathcal{C}(M), k=1, \ldots$, such that

$$
\limsup _{k \rightarrow \infty} \Psi\left(\mathrm{D}^{\mathrm{s}} u_{k}\right) \leq \sum_{i=1}^{m} \psi\left(a_{i}, b_{i}\right) .
$$

We refer to Remark 5.3 for a mild condition on the sequence $\left(a_{i}, b_{i}\right)$ that guarantees that the lim sup in (36) strengthens to lim and the inequality sign to the equality sign.

Proof. We shall first construct the sequence $u_{k}$ for the particular case when $M=$ $a \otimes b$ is a dyad and then superimpose the sequences corresponding to the dyads $a_{i} \otimes b_{i}, i=1, \ldots, m$, to obtain the general case. Thus, let $(a, b) \in \boldsymbol{D}_{n}$ and construct a sequence $u_{k} \in \mathcal{C}(a \otimes b), k=1, \ldots$, such that

$$
\lim _{k \rightarrow \infty} \Psi\left(\mathrm{D}^{\mathrm{s}} u_{k}\right)=\psi(a, b) .
$$

Introduce the sets

$$
C_{k}=\left(1-k^{-2}\right) Q, \quad L_{l}=\left(1-(l+1)^{-2}\right) Q \backslash\left(1-l^{-2}\right) Q,
$$

$k, l \in N$, and observe that

$$
Q=C_{k} \cup \bigcup_{l=k}^{\infty} L_{l}
$$


with mutually disjoint summands for any $k \in N$. Here the product $t S$ of a set $S \subset \boldsymbol{R}^{n}$ and a real number $t$ is defined by $t S=\{t x: x \in S\}$. Equation (39) presents a decomposition of $Q$ into the main set $C_{k}$, which is a large subset of $Q$ for large $k$, while $L_{k}, L_{k+1}, \ldots$ present infinitely many rectangular layers filling the gap $Q \backslash C_{k}$ and becoming more and more refined towards the boundary of $Q$.

We use these sets $C_{k}, L_{k}, L_{k+1}, \ldots$ to define a sequence of scalar functions $\varphi_{k}: Q \rightarrow \boldsymbol{R}, k=2, \ldots$, by

$$
\varphi_{k}(x)= \begin{cases}(k-1)^{-2}\left\langle(k-1)^{2} x \cdot b\right\rangle & \text { if } x \in C_{k}, \\ l^{-2}\left\langle l^{2} x \cdot b\right\rangle & \text { if } x \in L_{l} \text { for some } l \geq k .\end{cases}
$$

Let us use Remark 4.3 to prove that $\varphi_{k} \in B V(Q, \boldsymbol{R})$. Clearly, $\varphi_{k}$ is a piecewise constant function in the sense of that remark. Using (25a), one finds that $x \cdot b-1 \leq$ $\varphi_{k}(x) \leq x \cdot b$; hence, $\left|\varphi_{k}\right|$ is bounded on $Q$ and thus $\varphi_{k} \in L^{1}(Q, \boldsymbol{R})$. It remains to verify (32). Let us show that in the present case (32) reads

$$
\int_{J\left(\varphi_{k}\right)}\left|\llbracket \varphi_{k} \rrbracket\right| d \mathcal{A}<\infty
$$

where

$$
J\left(\varphi_{k}\right)=C_{k}^{\circ} \cup \bigcup_{l=k}^{\infty}\left(L_{l}^{\circ} \cup L_{l}^{\partial}\right)
$$

is the jump set, with

$$
\begin{aligned}
& C_{k}^{\circ}=\left\{x \in C_{k}: k^{2} x \cdot b \in Z\right\}, \\
& L_{l}^{\circ}=\left\{x \in L_{l}: l^{2} x \cdot b \in Z\right\}, \quad L_{l}^{\partial}=\left(1-l^{-2}\right) \partial Q,
\end{aligned}
$$

and on $J\left(\varphi_{k}\right)$

$$
\llbracket \varphi_{k} \rrbracket v_{\varphi_{k}}= \begin{cases}(k-1)^{-2} b & \text { on } C_{k}^{\circ}, \\ l^{-2} b & \text { on } L_{l}^{\circ} \text { where } l \geq k, \\ \eta_{l} v_{k} & \text { on } L_{l}^{\partial} \text { where } l \geq k\end{cases}
$$

is the jump and normal to the jump set, with

$$
\eta_{l}(x)=l^{-2}\left\langle l^{2} x \cdot b\right\rangle-(l-1)^{-2}\left\langle(l-1)^{2} x \cdot b\right\rangle
$$

and with $v_{k}$ denoting the outer normal to the scaled cube $\left(1-k^{-2}\right) Q$. Equations (42) and (45) follow from the identities given in Remark 4.3. One has to enumerate the regions of constancy of $\varphi_{k}$ in an arbitrary way to obtain the system of sets $\Omega_{\alpha}$, $\alpha=1, \ldots$, and use the formulas of that remark. The details are left to the reader. This establishes the equivalence of the inequalities (32) and (41). To prove that 
(41) really holds, one finds from (45) that

$$
\int_{J\left(u_{k}\right)}\left|\llbracket \varphi_{k} \rrbracket\right| d \mathcal{A}=(k-1)^{-2} \mathcal{A}\left(C_{k}^{\circ}\right)+\sum_{l=k}^{\infty} l^{-2} \mathcal{A}\left(L_{l}^{\circ}\right)+\sum_{l=k}^{\infty} \int_{L_{l}^{\partial}}\left|\eta_{l}\right| d \mathcal{A} .
$$

We estimate the terms $\mathcal{A}\left(C_{k}^{\circ}\right), \mathcal{A}\left(L_{l}^{\circ}\right)$ and $\int_{L_{l}^{\partial}}\left|\eta_{l}(x)\right| d \mathcal{A}$ as follows. First, prove that

$$
\begin{aligned}
\left|\mathcal{A}\left(C_{k}^{\circ}\right)-(k-1)^{2} \mathcal{L}^{n}\left(C_{k}\right)\right| & \leq 2 n, \\
\left|\mathcal{A}\left(L_{l}^{\circ}\right)-l^{2} \mathcal{L}^{n}\left(L_{l}\right)\right| & \leq 4 n
\end{aligned}
$$

and hence

$$
\mathcal{A}\left(C_{k}^{\circ}\right) \leq 2 n+(k-1)^{2} \mathcal{L}^{n}\left(C_{k}\right), \quad \mathcal{A}\left(L_{l}^{\circ}\right) \leq 4 n+l^{2} \mathcal{L}^{n}\left(L_{l}\right) .
$$

Let us prove (48b); the proof of (48a) is similar. Let $\omega: L_{l} \rightarrow \boldsymbol{R}$ be defined by

$$
\omega(x)=l^{2} x \cdot b-\left\langle l^{2} x \cdot b\right\rangle, \quad x \in L_{l} .
$$

Then $\omega \in B V\left(L_{l}, \boldsymbol{R}\right), \mathrm{D} \omega=l^{2} b-b \mathcal{A}\left\llcorner L_{l}^{\circ}\right.$, and hence the Gauss-Green theorem yields

$$
\mathrm{D} \omega\left(L_{l}\right)=l^{2} \mathcal{L}^{n}\left(L_{l}\right) b-b \mathcal{A}\left(L_{l}^{\circ}\right)=\int_{\partial L_{l}} \omega v_{L_{l}} d \mathcal{A},
$$

from which

$$
\left|m \mathcal{L}^{n}\left(L_{l}\right)-\mathcal{A}\left(L_{l}^{\circ}\right)\right| \leq \int_{\partial L_{l}}|\omega| d \mathcal{A}
$$

We now observe that $|\omega| \leq 1$ on $\partial L_{l}$ and $\partial L_{l}=L_{l+1}^{\partial} \cup L_{l}^{\partial}$. Thus,

$$
\int_{\partial L_{l}}|\omega| d \mathcal{A} \leq \mathcal{A}\left(L_{l+1}^{\partial}\right)+\mathcal{A}\left(L_{l}^{\partial}\right) \leq 4 n
$$

since, elementarily, $\mathcal{A}\left(L_{l+1}^{\partial}\right) \leq 2 n$ and $\mathcal{A}\left(L_{l}^{\partial}\right) \leq 2 n$. Thus, we have (48b). Next prove that

$$
\left|\eta_{l}(x)\right| \leq 2(l-1)^{-2} \quad \text { on } L_{l}^{\partial} .
$$

Indeed, writing

$$
\left|\eta_{l}(x)\right|=\left|\left(l^{-2}\left\langle l^{2} x \cdot b\right\rangle-x \cdot b\right)-\left((l-1)^{-2}\left\langle(l-1)^{2} x \cdot b\right\rangle-x \cdot b\right)\right|,
$$

using the triangle inequality and the inequality (26) twice, with $t=x \cdot b$ and $k=l^{2}$ and $k=(l-1)^{2}$, one obtains

$$
\left|\eta_{l}(x)\right| \leq l^{-2}+(l-1)^{-2} \leq 2(l-1)^{-2}
$$

and hence

$$
\int_{L_{l}^{\partial}}\left|\eta_{l}\right| d \mathcal{A} \leq 2(l-1)^{-2} \mathcal{A}\left(L_{l}^{\partial}\right) \leq 4 n(l-1)^{-2} .
$$


The estimates (49) and (51) and the formula (47) provide

$$
\begin{aligned}
\int_{J\left(u_{k}\right)}\left|\llbracket \varphi_{k} \rrbracket\right| d \mathcal{A} & \leq 2 n(k-1)^{-2}+\mathcal{L}^{n}\left(C_{k}\right) \\
& +\sum_{l=k}^{\infty}\left(4 n l^{-2}+\mathcal{L}^{n}\left(L_{l}\right)\right)+\sum_{l=k}^{\infty} 4 n(l-1)^{-2} \\
& \leq 1+2 n(k-1)^{-2}+8 n \sum_{l=k}^{\infty}(l-1)^{-2}<\infty,
\end{aligned}
$$

where we have used

$$
\mathcal{L}^{n}\left(C_{k}\right)+\sum_{l=k}^{\infty} \mathcal{L}^{n}\left(L_{l}\right)=\mathcal{L}^{n}(Q)=1 .
$$

Thus, we have (41); hence, $\varphi_{k} \in B V(\Omega, \boldsymbol{R})$ for every $k$ and

$$
\mathrm{D} \varphi_{k}=\llbracket \varphi_{k} \rrbracket v_{\varphi_{k}} \mathcal{A}\left\llcorner J\left(\varphi_{k}\right)\right.
$$

and

$$
\nabla \varphi_{k}=0
$$

Finally, note that the boundary trace $\varphi_{k}^{\partial}$ of $\varphi_{k}$ on $\partial Q$ satisfies

$$
\varphi_{k}^{\partial}(x)=x \cdot b \quad \text { for every } x \in \partial Q .
$$

While a rigorous proof of this can be given by using the essential limit of $\varphi_{k}$ at $x \in Q$, we here only note that the definition of $\varphi_{k}$ yields that

$$
\lim _{j \rightarrow \infty} \varphi_{k}\left(x_{j}\right)=x \cdot b
$$

for any $x \in \partial Q$ and any sequence $x_{j} \in Q$ converging to $x$. For this it suffices to note that in view of (39) one finds that $x_{j}$ must belong to some $L_{l}$ for some $l=l(j) \geq k$. The limit $x_{j} \rightarrow x$ then implies that $l(k) \rightarrow \infty$, and then the definition (40) and the formula (27) provide (54).

We define the sequence $u_{k}: Q \rightarrow \boldsymbol{R}^{n}, k=2, \ldots$, by

$$
u_{k}(x)=a \varphi_{k}(x)
$$

for every $x \in Q$. By $\varphi_{k} \in S B V(Q, \boldsymbol{R})$ and by (52) and (53), we have $u_{k} \in \mathcal{C}(a \otimes b)$. Further, $\llbracket u_{k} \rrbracket=\llbracket \varphi_{k} \rrbracket a \otimes v_{\varphi_{k}} ;$ consequently, by (45),

$$
\psi\left(\llbracket u_{k} \rrbracket, v_{u_{k}}\right)= \begin{cases}(k-1)^{-2} \psi(a, b) & \text { on } C_{k}^{\circ}, \\ l^{-2} \psi(a, b) & \text { on } L_{l}^{\circ} \text { for any } l \geq k, \\ \psi\left(\eta_{l} a, v_{l}\right) & \text { on } L_{l}^{\partial} \text { for any } l \geq k\end{cases}
$$


and hence

$$
\Psi\left(\mathrm{D}^{\mathrm{s}} u_{k}\right)=\int_{J\left(u_{k}\right)} \psi\left(\llbracket u_{k} \rrbracket, v_{u_{k}}\right) d \mathcal{A}=(k-1)^{-2} \psi(a, b) \mathcal{A}\left(C_{k}^{\circ}\right)+\rho_{k},
$$

where

$$
\rho_{k}=\sum_{l=k}^{\infty} l^{-2} \psi(a, b) \mathcal{A}\left(L_{l}^{\circ}\right)+\sum_{l=k}^{\infty} \int_{L_{l}^{\partial}} \psi\left(\eta_{l} a, v_{l}\right) d \mathcal{A} .
$$

Dividing (48a) by $(k-1)^{2}$, we obtain

$$
(k-1)^{-2} \mathcal{A}\left(C_{k}^{\circ}\right) \rightarrow 1
$$

since $\mathcal{L}^{n}\left(C_{k}\right) \rightarrow 1$. Using (6), we obtain that the nonnegative number $\rho_{k}$ is bounded by (a constant multiple of) the quantity

$$
\begin{aligned}
d_{k} & =\sum_{l=k}^{\infty} l^{-2} \mathcal{A}\left(L_{l}^{\circ}\right)+\sum_{l=k}^{\infty} \int_{L_{l}^{\partial}}\left|\eta_{l}\right| d \mathcal{A} \\
& \leq \sum_{l=k}^{\infty} \mathcal{L}^{n}\left(L_{l}\right)+2 n(k-1)^{-2}+4 n \sum_{l=k}^{\infty}(l-1)^{-2} \\
& \leq k^{-2}+2 n(k-1)^{-2}+4 n \sum_{l=k}^{\infty}(l-1)^{-2}
\end{aligned}
$$

and hence $\rho_{k} \rightarrow 0$. Equations (55) and (56) then yield (37).

We now complete the proof in the general case. By the preceding part of the proof, for each $i \in\{1, \ldots, m\}$, there exists a sequence $u_{k}^{i} \in \mathcal{C}\left(a_{i} \otimes b_{i}, 0\right), k=1, \ldots$, such that

$$
\Psi\left(\mathrm{D}^{\mathrm{s}} u_{k}^{i}\right) \rightarrow \psi\left(a_{i}, b_{i}\right)
$$

as $k \rightarrow \infty$. Define $u_{k}:=\sum_{i=1}^{m} u_{k}^{i}$ for every $k$. By (28),

$$
\Psi\left(\mathrm{D}^{\mathrm{s}} u_{k}\right) \leq \sum_{i=1}^{m} \Psi\left(\mathrm{D}^{\mathrm{s}} u_{k}^{i}\right)
$$

Hence,

$$
\limsup _{k \rightarrow \infty} \Psi\left(\mathrm{D}^{\mathrm{s}} u_{k}\right) \leq \lim _{k \rightarrow \infty} \sum_{i=1}^{m} \Psi\left(\mathrm{D}^{\mathrm{s}} u_{k}^{i}\right)=\sum_{i=1}^{m} \psi\left(a_{i}, b_{i}\right)
$$

by (57).

5.3. Remark. If the sequence $\left(a_{i}, b_{i}\right)$ satisfies the condition

$$
b_{i} \neq b_{j} \quad \text { and } \quad b_{i} \neq-b_{j} \quad \text { whenever } 1 \leq i<j \leq m,
$$


then the sequence $u_{k}$ can be chosen as to satisfy, instead of the inequality (36), the equality

$$
\lim _{k \rightarrow \infty} \Psi\left(\mathrm{D}^{\mathrm{s}} u_{k}\right)=\sum_{i=1}^{m} \psi\left(a_{i}, b_{i}\right) .
$$

Indeed, the inspection of the proof of Lemma 5.2 shows that the source of the inequality (36) is the subadditivity in (58) which cannot be replaced by the equality unless the discontinuity sets $J\left(u_{i}\right)$ pairwise intersect on a set of null $\mathcal{A}$ measure (see Proposition 4.1). Condition (59) guarantees that. However, inequality (36) suffices for our purposes.

5.4. Proposition. $\Phi_{2} \geq \Phi_{3} \geq \Phi_{4}$.

Proof. To prove $\Phi_{2} \geq \Phi_{3}$, we take any sequence $\left(a_{i}, b_{i}\right) \in \boldsymbol{D}_{n}, i=1, \ldots, m$, such that $\sum_{i=1}^{m} a_{i} \otimes b_{i}=M$ and consider the infimum as in the definition of $\Phi_{2}$ in (16). Hence, for the given sequence $\left(a_{i}, b_{i}\right) \in \boldsymbol{D}_{n}$, we construct a sequence of maps $u_{k} \in \mathcal{C}(M), k=1, \ldots$, as in Lemma 5.2. Then

$$
\Phi_{3}(M) \leq \Psi\left(\mathrm{D}^{\mathrm{s}} u_{k}\right)
$$

by the definition of $\Phi_{3}$. Letting $k \rightarrow \infty$ and using (36), we obtain

$$
\Phi_{3}(M) \leq \sum_{i=1}^{m} \psi\left(a_{i}, b_{i}\right) .
$$

Taking the infimum over all sequences $a_{i}$ and $b_{i}$, one obtains from the definition of $\Phi_{2}$ the inequality $\Phi_{3}(M) \leq \Phi_{2}(M)$. The inequality $\Phi_{3} \geq \Phi_{4}$ is immediate.

5.5. Proposition. $\Phi_{4}=\Phi_{1}$.

Proof. We seek to prove that $\Phi_{4}$ is the biggest subadditive function satisfying $\Phi_{4}(a \otimes b) \leq \psi(a, b)$ for any $(a, b) \in \boldsymbol{D}_{n}$. To prove the subadditivity of $\Phi_{4}$, let $A, B \in$ Lin and $u \in \mathcal{B}(A)$ and $v \in \mathcal{B}(B)$. Proposition 4.1 and (17) yield $u+v \in$ $\mathcal{B}(A+B)$ and

$$
\Phi_{4}(A+B) \leq \Psi\left(\mathrm{D}^{\mathrm{s}} u+\mathrm{D}^{\mathrm{s}} v\right) \leq \Psi\left(\mathrm{D}^{\mathrm{s}} u\right)+\Psi\left(\mathrm{D}^{\mathrm{s}} v\right) .
$$

Taking the infimum over all $u$ and $v$ then gives the subadditivity

$$
\Phi_{4}(A+B) \leq \Phi_{4}(A)+\Phi_{4}(B) .
$$

Next we note that the biggest subadditive function $\Theta$ such that

$$
\Theta(a \otimes b) \leq \psi(a, b)
$$

for any $(a, b) \in \boldsymbol{D}_{n}$ is automatically positively homogeneous; thus, it suffices to prove the maximality of $\Phi_{4}$ among all subadditive and positively homogeneous functions satisfying (60). Thus, let $\Theta$ be such a function and let $M \in \operatorname{Lin}$ and 
$u \in \mathcal{B}(M)$. Then by (60) and by Jensen's inequality for positively homogeneous subadditive functions,

$$
\begin{aligned}
\Psi\left(\mathrm{D}^{\mathrm{s}} u\right) & :=\int_{J(u)} \psi\left(\llbracket u \rrbracket, v_{u}\right) d \mathcal{A} \\
& \geq \int_{J(u)} \Theta\left(\llbracket u \rrbracket \otimes v_{u}\right) d \mathcal{A} \\
& \geq \Theta\left(\int_{J(u)} \llbracket u \rrbracket \otimes v_{u} d \mathcal{A}\right) .
\end{aligned}
$$

We now combine the boundary condition $u=w_{M}$ on $\partial Q$ and relation $\int_{Q} \nabla u d \mathcal{L}^{n}=0$ with the Gauss-Green theorem to obtain

$$
\begin{aligned}
\int_{J(u)} \llbracket u \rrbracket \otimes v_{u} d \mathcal{A} & =\int_{J(u)} \llbracket u \rrbracket \otimes v_{u} d \mathcal{A}+\int_{Q} \nabla u d \mathcal{L}^{n} \\
& =\int_{Q} 1 d \mathrm{D} u \\
& =\int_{\partial Q} M x \otimes v_{Q} d \mathcal{A}=M .
\end{aligned}
$$

Thus, (61) yields

$$
\Psi\left(\mathrm{D}^{\mathrm{s}} u\right) \geq \Theta(M) .
$$

Taking the infimum over all $u \in \mathcal{B}(M)$, we obtain $\Phi_{4}(M) \geq \Theta(M)$.

This proves $\Phi_{1}=\Phi_{2}=\Phi_{3}=\Phi_{4}$. We define the function $\Phi$ by $\Phi=\Phi_{1}$.

\section{Completion of the proof of Theorem 2.3}

For this section, we put, for every $(a, b) \in \boldsymbol{D}_{n}$,

$$
\mathrm{C}(a, b):=\left\{u \in \operatorname{SBV}\left(Q_{b}\right): u=z_{a, b} \text { on } \partial Q_{b}, \nabla u=0 \text { on } Q_{b}\right\}
$$

and denote by $\Phi_{5}(a, b)$ the infimum in (19). We then extend $\Phi_{5}$ to $\boldsymbol{R}^{n} \times \boldsymbol{R}^{n}$ by homogeneity in the second variable.

6.1. Proposition. We have $H(A, B)=\Phi(A-B)$ for every $A, B \in$ Lin.

Proof. We employ Theorem 2.2 and the definition of $\Phi$ in (18). Invoking (11), we take any $u \in S B V(Q)$ satisfying $u=w_{A}$ on $\partial Q$, and $\int_{Q} \nabla u d \mathcal{L}^{n}=B$. Then $v$, given by $v(x)=u(x)-B x, x \in Q$, satisfies $v \in \mathcal{B}(A-B)$ and $\Psi\left(\mathrm{D}^{\mathrm{s}} u\right)=\Psi\left(\mathrm{D}^{\mathrm{s}} v\right)$.

6.2. Lemma. We have $\Phi_{5}(a, b) \leq \Phi(a \otimes b)$ for every $(a, b) \in \boldsymbol{D}_{n}$.

Proof. Let $(a, b) \in \boldsymbol{D}_{n}$, and let $\left(a_{i}, b_{i}\right) \in \boldsymbol{D}_{n}, i=1, \ldots, m$, be a sequence satisfying

$$
a \otimes b=\sum_{i=1}^{m} a_{i} \otimes b_{i} .
$$


Our goal is to construct a sequence $u_{k} \in \mathrm{C}(a, b), k=1, \ldots$, such that

$$
\limsup _{k \rightarrow \infty} \int_{J\left(u_{k}\right)} \psi\left(\llbracket u_{k} \rrbracket, v_{u_{k}}\right) d \mathcal{A} \leq \sum_{i=1}^{m} \psi\left(a_{i}, b_{i}\right) .
$$

To define $u_{k}$, let

$$
P=\left\{x \in \boldsymbol{R}^{n}: x \cdot b=0\right\}
$$

be the plane through the origin perpendicular to $b$, let $\Pi$ be the projection from $\boldsymbol{R}^{n}$ onto $P$, let

$$
F=P \cap Q_{b},
$$

and put

$$
B_{k}=\left\{x \in \boldsymbol{R}^{n}: \Pi(x) \in\left(1-k^{-1}\right) F, 0 \leq x \cdot b<k^{-1}\right\}
$$

for any $k \in N$. Define $u_{k}: Q_{b} \rightarrow \boldsymbol{R}^{n}$ by

$$
u_{k}(x)= \begin{cases}v_{k}(x) & \text { if } x \in B_{k}, \\ z_{a, b}(x) & \text { else, }\end{cases}
$$

$x \in Q_{b}$, where

$$
v_{k}(x)=\sum_{i=1}^{m} k^{-1} a_{i}\left\langle k^{2} x \cdot b_{i}\right\rangle \quad \text { for any } x \in \boldsymbol{R}^{n} \text { and } k \in N .
$$

Employing Remark 4.2, we see that $u_{k} \in S B V\left(Q_{b}\right)$; furthermore, clearly, $u_{k}=z_{a, b}$ on $\partial Q_{b}$ and $\nabla u_{k}=0$ on $Q_{b}$; hence, $u_{k} \in \mathrm{C}(a, b)$.

We proceed to prove (63). We have

$$
J\left(u_{k}\right)=N_{k} \cup M_{k} \cup L_{k} \cup S_{k},
$$

where

$$
\begin{aligned}
N_{k} & =F \backslash\left(1-k^{-1}\right) F, \\
M_{k} & =\left\{x \in \partial B: 0<x \cdot b<k^{-1}\right\}, \\
S_{k} & =\left\{x \in \boldsymbol{R}^{n}: \Pi(x) \in\left(1-k^{-1}\right) F, x \cdot b=k^{-1}\right\}, \\
L_{k} & =\bigcup_{i=1}^{m} L_{k}^{i} \quad \text { where } L_{k}^{i}=\left\{x \in B_{k}: k^{2} x \cdot b_{i} \in Z\right\} .
\end{aligned}
$$

The jump of $u_{k}$ and the normal to the jump set are

$$
\llbracket u_{k} \rrbracket(x) v_{u_{k}}(x)= \begin{cases}k^{-1} \sum_{i=1}^{m} a_{i} \otimes b_{i} 1_{L_{k}^{i}}(x) & \text { if } x \in L_{k}, \\ a \otimes b & \text { if } x \in N_{k}, \\ \left(a-v_{k}(x)\right) \otimes v_{k} & \text { if } x \in M_{k}, \\ \left(a-v_{k}(x)\right) \otimes b & \text { if } x \in S_{k},\end{cases}
$$


$x \in J\left(u_{k}\right)$, where $v_{k}$ is the outer normal to $B_{k}$ and $1_{L_{k}^{i}}$ is the characteristic function of the set $L_{k}^{i}$. Hence, the subadditivity of $\psi$ in the first variable yields

$$
\int_{L_{k}} \psi\left(\llbracket u_{k} \rrbracket, v_{u_{k}}\right) d \mathcal{A} \leq k^{-1} \sum_{i=1}^{m} \psi\left(a_{i}, b_{i}\right) \mathcal{A}\left(L_{k}^{i}\right) ;
$$

consequently

$$
\begin{aligned}
\int_{J\left(u_{k}\right)} \psi\left(\llbracket u_{k} \rrbracket, v_{u_{k}}\right) d \mathcal{A} \leq k^{-1} \sum_{i=1}^{m} \psi\left(a_{i}, b_{i}\right) \mathcal{A}( & \left.L_{k}^{i}\right)+\psi(a, b) \mathcal{A}\left(N_{k}\right) \\
& \left.+\int_{M_{k}} \psi\left(a-v_{k}(x)\right), v_{k}\right) d \mathcal{A} \\
& \left.+\int_{S_{k}} \psi\left(a-v_{k}(x)\right), b\right) d \mathcal{A} .
\end{aligned}
$$

Let us now analyze the terms on the right-hand side of (67). Using the considerations as in the proof of Lemma 5.2 (see (48) and (49)), one finds that

$$
k^{-1} \mathcal{A}\left(L_{k}^{i}\right) \rightarrow 1
$$

as $k \rightarrow \infty$ for every $i=1, \ldots, m$. Thus,

$$
k^{-1} \sum_{i=1}^{m} \psi\left(a_{i}, b_{i}\right) \mathcal{A}\left(L_{k}^{i}\right) \rightarrow \sum_{i=1}^{m} \psi\left(a_{i}, b_{i}\right) .
$$

Further,

$$
\psi(a, b) \mathcal{A}\left(N_{k}\right) \rightarrow 0
$$

since, obviously,

$$
\mathcal{A}\left(N_{k}\right) \rightarrow 0
$$

Next note that, by (62) and (26),

$$
\begin{aligned}
\left|k a(x \cdot b)-v_{k}(x)\right| & =\left|k a(x \cdot b)-\sum_{i=1}^{m} k^{-1} a_{i}\left\langle k^{2} x \cdot b_{i}\right\rangle\right| \\
& =\left|k \sum_{i=1}^{m} a_{i}\left(x \cdot b_{i}\right)-k^{-2} a_{i}\left\langle k^{2} x \cdot b_{i}\right\rangle\right| \\
& \leq\left|k \sum_{i=1}^{m}\right| a_{i}||\left(x \cdot b_{i}\right)-k^{-2}\left\langle k^{2} x \cdot b_{i}\right\rangle|| \\
& \leq k^{-1} \sum_{i=1}^{m}\left|a_{i}\right| .
\end{aligned}
$$


Then if $x \in M_{k}$,

$$
\begin{aligned}
\left|a-v_{k}(x)\right| & \leq|a-k a(x \cdot b)|+\left|k a(x \cdot b)-v_{k}(x)\right| \\
& \leq|a|+k|a||x \cdot b|+k^{-1} \sum_{i=1}^{m}\left|a_{i}\right| \\
& \leq|a|+|a|+k^{-1} \sum_{i=1}^{m}\left|a_{i}\right|
\end{aligned}
$$

since $k|x \cdot b| \leq 1$ on $M_{k}$. Thus, $\left|a-v_{k}(x)\right| \leq c<\infty$ for any $x \in M_{k}$ and any $k=1, \ldots$ A combination with $(6)$ and

$$
\mathcal{A}\left(M_{k}\right) \rightarrow 0
$$

then provides

$$
\left.\int_{M_{k}} \psi\left(a-v_{k}(x)\right), v_{k}\right) d \mathcal{A} \rightarrow 0 .
$$

Similarly, if $x \in S_{k}$, then $k x \cdot b=1$ and hence

$$
\left|a-v_{k}(x)\right| \leq\left|k a(x \cdot b)-v_{k}(x)\right| \leq k^{-1} \sum_{i=1}^{m}\left|a_{i}\right| \rightarrow 0 .
$$

Thus, (6) yields

$$
\left.\int_{S_{k}} \psi\left(a-v_{k}(x)\right), b\right) d \mathcal{A} \rightarrow 0
$$

since $\mathcal{A}\left(S_{k}\right) \leq 1$ for all $k$. Consequently, a combination of (67) with (68)-(71) provides (63) and hence the definition of $\Phi_{5}$ gives

$$
\Phi_{5}(a, b) \leq \sum_{i=1}^{m} \psi\left(a_{i}, b_{i}\right)
$$

for any sequence $\left(a_{i}, b_{i}\right)$ satisfying (62). Taking the infimum of the right-hand side over all such sequences and using the definition of $\Phi_{2} \equiv \Phi$, we obtain the assertion.

6.3. Lemma. We have $\Phi_{5}(a, b) \geq \Phi(a \otimes b)$ for every $(a, b) \in \boldsymbol{D}_{n}$.

Proof. Let $u \in \mathrm{C}(a, b)$. Then, by Jensen's inequality,

$$
\begin{aligned}
\int_{J(u)} \psi\left(\llbracket u \rrbracket, v_{u}\right) d \mathcal{A} & \geq \int_{J(u)} \Phi\left(\llbracket u \rrbracket \otimes v_{u}\right) d \mathcal{A} \\
& \geq \Phi\left(\int_{J(u)} \llbracket u \rrbracket \otimes v_{u} d \mathcal{A}\right) \\
& =\Phi(a \otimes b)
\end{aligned}
$$


since the boundary condition $u=z_{a, b}$ on $\partial Q_{b}$ implies

$$
\int_{J(u)} \llbracket u \rrbracket \otimes v_{u} d \mathcal{A}=a \otimes b .
$$

That is, we have

$$
\int_{J(u)} \psi\left(\llbracket u \rrbracket, v_{u}\right) d \mathcal{A} \geq \Phi(a \otimes b)
$$

for every $u \in \mathrm{C}(a, b)$. Taking the infimum, we obtain $\Phi_{5}(a, b) \geq \Phi(a \otimes b)$.

6.4. Proposition. We have $h(a, b)=\Phi(a \otimes b)$ for every $(a, b) \in \boldsymbol{D}_{n}$.

Proof. This follows immediately from (12) and (19).

This completes the proof of Theorem 2.3.

\section{Derivation of the examples}

Derivation of Example 2.5 and (21). We consider $\psi_{|\cdot|}(a, b)=|a \cdot b|$ first and prove (21a). Clearly, the function $\Theta(M)=|\operatorname{tr} M|$ is a subadditive function satisfying (14) with $\psi=\psi_{|\cdot|}$ and hence (16) gives $\Phi_{|\cdot|}(M) \geq|\operatorname{tr} M|$ for any $M \in$ Lin. To prove the opposite inequality, we note that the definition (15) of $\Phi_{|\cdot|}$ gives

$$
\psi_{|\cdot|}(a, b)=\Theta(a \otimes b) \leq \Phi_{|\cdot|}(a \otimes b) \leq \psi_{|\cdot|}(a, b)
$$

for every $(a, b) \in \boldsymbol{D}_{n}$ and hence

$$
\Phi_{|\cdot|}(a \otimes b)=|a \cdot b| \quad \text { and in particular } \quad \Phi_{|\cdot|}(a \otimes b)=0 \quad \text { if } a \cdot b=0,
$$

which determines $\Phi_{|\cdot|}$ on tensor products $a \otimes b$. As a consequence, if $N \in$ Lin can be written as

$$
N=\sum_{i=1}^{m} a_{i} \otimes b_{i}
$$

where $\left(a_{i}, b_{i}\right) \in \boldsymbol{R}^{n} \times \boldsymbol{R}^{n}, i=1, \ldots, m$, where

$$
a_{i} \cdot b_{i}=0 \text { for all } i=1, \ldots, m,
$$

then $\Phi_{|\cdot|}(N)=0$ since

$$
0 \leq \Phi_{|\cdot|}(N) \leq \sum_{i=1}^{m} \Phi_{|\cdot|}\left(a_{i} \otimes b_{i}\right) \leq \sum_{i=1}^{m} \psi\left(a_{i}, b_{i}\right)=\sum_{i=1}^{m}\left|a_{i} \cdot b_{i}\right|=0 .
$$

To determine $\Phi_{|\cdot|}$ on a general $M \in$ Lin, we write $M=A+W$ where $A$ and $W$ are the symmetric and skew parts of $M$. Let $e_{1}, \ldots, e_{n}$ be an orthonormal basis of eigenvectors of $A$ with the eigenvalues $\lambda_{i}$; hence, $A=\sum_{i=1}^{n} \lambda_{i} e_{i} \otimes e_{i}$. Then

$$
M=B+N
$$


where

$$
\begin{aligned}
& B=(\operatorname{tr} M) e_{1} \otimes e_{1}, \\
& N=W+\sum_{i=2}^{n} \lambda_{i}\left(e_{i} \otimes e_{1}-e_{1} \otimes e_{i}-\left(e_{1}+e_{i}\right) \otimes\left(e_{1}-e_{i}\right)\right) .
\end{aligned}
$$

Since $W$ is a linear combination of the dyads $e_{i} \otimes e_{j}, 1 \leq i \neq j \leq n$, one sees that $N$ is of the form (72)-(73) and hence $\Phi_{|\cdot|}(N)=0$; consequently

$$
\Phi_{|\cdot|}(M) \leq \Phi_{|\cdot|}(B)+\Phi_{|\cdot|}(N)=\Phi_{|\cdot|}(B)=\psi\left((\operatorname{tr} M) e_{1}, e_{1}\right)=|\operatorname{tr} M| .
$$

Equations 13 complete the proof of (21a).

To prove the two equations in (21b), we employ (21a) and (21b) as follows. One has $\psi_{ \pm}(a, b)=\frac{1}{2}(|a \cdot b| \pm a \cdot b)$, and hence, if $\left(a_{i}, b_{i}\right) \in \boldsymbol{D}_{n}$ and $M \in$ Lin satisfy $\sum_{i=1}^{m} a_{i} \otimes b_{i}=M$, then

$$
\sum_{i=1}^{m} \psi_{ \pm}\left(a_{i}, b_{i}\right)=\frac{1}{2}\left(\sum_{i=1}^{m} \psi_{|\cdot|}\left(a_{i}, b_{i}\right) \pm \operatorname{tr} M\right) .
$$

Taking the infimum as in (16) and using the above evaluation of $\Phi_{|\cdot|}$ gives

$$
\Phi_{ \pm}(M)=\frac{1}{2}\left(\Phi_{|\cdot|}(M) \pm \operatorname{tr} M\right)=\frac{1}{2}(|\operatorname{tr} M| \pm \operatorname{tr} M)=\{\operatorname{tr} M\}_{ \pm},
$$

which is (21b).

Derivation of Example 2.6 and (23). The function $\Theta(M)=\left|M^{\mathrm{T}} p\right|$ is a subadditive function satisfying (14), and we obtain in the same way as in the proof of Example 2.5 that $\Phi(M) \geq\left|M^{\mathrm{T}} p\right|$ for any $M \in \operatorname{Lin}$ and

$$
\Phi(a \otimes b)=|a \cdot p| \quad \text { and in particular } \quad \Phi(a \otimes b)=0 \quad \text { if } a \cdot p=0 .
$$

To prove $\Phi(M) \leq\left|M^{\mathrm{T}} p\right|$, we assume without loss of generality that $|p|=1$ and let $\left\{p, e_{2}, \ldots e_{n}\right\}$ be any orthonormal basis. In view of $\mathbf{1}=p \otimes p+\sum_{i=2}^{n} e_{i} \otimes e_{i}$,

$$
M=\mathbf{1} M=p \otimes M^{\mathrm{T}} p+\sum_{i=2}^{n} e_{i} \otimes M^{\mathrm{T}} e_{i} ;
$$

normalizing the second members of the dyads, we obtain

$$
M=\left|M^{\mathrm{T}} p\right| p \otimes \operatorname{sgn}\left(M^{\mathrm{T}} p\right)+\sum_{i=2}^{n}\left|M^{\mathrm{T}} e_{i}\right| e_{i} \otimes \operatorname{sgn}\left(M^{\mathrm{T}} e_{i}\right) .
$$

The subadditivity of $\Phi$ provides

$$
\Phi(M) \leq \Phi\left(\left|M^{\mathrm{T}} p\right| p \otimes \operatorname{sgn}\left(M^{\mathrm{T}} p\right)\right)+\sum_{i=2}^{n} \Phi\left(\left|M^{\mathrm{T}} e_{i}\right| e_{i} \otimes \operatorname{sgn}\left(M^{\mathrm{T}} e_{i}\right)\right)=\left|M^{\mathrm{T}} p\right|
$$

by (74). Thus, $\Phi(M) \leq\left|M^{\mathrm{T}} p\right|$ and the proof of (23) is complete. 


\section{Acknowledgments}

I thank David Owen for a detailed discussion on the previous version of this work. His stimulating questions contributed to the final version of the results presented here. Also, David Owen and the two anonymous reviewers pointed out to me that it is possible to replace my original coercivity assumption on $\psi$ in Assumption 2.1(ii) by mere nonnegativity, which is necessary to cover Examples 2.5 and 2.6 and which is possible by [Choksi and Fonseca 1997, Remark 3.3]. I am also thankful for the support of the institutional research plan RVO 67985840.

\section{References}

[Ambrosio et al. 2000] L. Ambrosio, N. Fusco, and D. Pallara, Functions of bounded variation and free discontinuity problems, Clarendon, New York, 2000.

[Baía et al. 2011] M. Baía, J. Matias, and P. M. Santos, "A survey on structured deformations", São Paulo J. Math. Sci. 5:2 (2011), 185-201.

[Baía et al. 2012] M. Baía, J. Matias, and P. M. Santos, "A relaxation result in the framework of structured deformations in a bounded variation setting", Proc. Roy. Soc. Edinburgh A 142:2 (2012), 239-271.

[Barroso et al. 2017] A. C. Barroso, J. Matias, M. Morandotti, and D. R. Owen, "Explicit formulas for relaxed disarrangement densities arising from structured deformations", Math. Mech. Complex Syst. 5:2 (2017), 163-189.

[Choksi and Fonseca 1997] R. Choksi and I. Fonseca, "Bulk and interfacial energy densities for structured deformations of continua”, Arch. Rational Mech. Anal. 138:1 (1997), 37-103.

[Del Piero 2001] G. Del Piero, “The energy of a one-dimensional structured deformation", Math. Mech. Solids 6:4 (2001), 387-408.

[Del Piero 2004] G. Del Piero, "Foundations of the theory of structured deformations", pp. 125-175 in Multiscale modeling in continuum mechanics and structured deformations, edited by G. Del Piero and D. R. Owen, International Centre for Mechanical Sciences 447, Springer, Vienna, 2004.

[Del Piero and Owen 1993] G. Del Piero and D. R. Owen, "Structured deformations of continua", Arch. Rational Mech. Anal. 124:2 (1993), 99-155.

[Del Piero and Owen 1995] G. Del Piero and D. R. Owen, "Integral-gradient formulae for structured deformations", Arch. Rational Mech. Anal. 131:2 (1995), 121-138.

[Del Piero and Owen 2004] G. Del Piero and D. R. Owen (editors), Multiscale modeling in continuum mechanics and structured deformations, International Centre for Mechanical Sciences 447, Springer, Vienna, 2004.

[Evans and Gariepy 1992] L. C. Evans and R. F. Gariepy, Measure theory and fine properties of functions, CRC, Boca Raton, FL, 1992.

[Federer 1969] H. Federer, Geometric measure theory, Grundlehren der math. Wissenschaften 153, Springer, Berlin, 1969.

[Hille and Phillips 1957] E. Hille and R. S. Phillips, Functional analysis and semi-groups, rev. ed., AMS Colloquium Publications 31, American Mathematical Society, Providence, RI, 1957.

[Owen and Paroni 2015] D. R. Owen and R. Paroni, "Optimal flux densities for linear mappings and the multiscale geometry of structured deformations", Arch. Ration. Mech. Anal. 218:3 (2015), $1633-1652$. 
[Reshetnyak 1968] Yu. G. Reshetnyak, "The weak convergence of completely additive vector-valued set functions”, Siberian Math. J. 9:6 (1968), 1039-1045.

[Šilhavý 2015] M. Šilhavý, "On the approximation theorem for structured deformations from $B V(\Omega)$ ", Math. Mech. Complex Syst. 3:1 (2015), 83-100.

[Ziemer 1989] W. P. Ziemer, Weakly differentiable functions: Sobolev spaces and functions of bounded variation, Graduate Texts in Math. 120, Springer, Berlin, 1989.

Received 25 Oct 2016. Revised 7 Mar 2017. Accepted 16 May 2017.

MIROSLAV ŠILHAVÝ: silhavy@math.cas.cz

Institute of Mathematics, Academy of Sciences of the Czech Republic, Žitná 25, 11567 Pragua 1, Czech Republic 



\section{Guidelines for Authors}

Authors may submit manuscripts in PDF format on-line at the submission page.

Originality. Submission of a manuscript acknowledges that the manuscript is original and and is not, in whole or in part, published or under consideration for publication elsewhere. It is understood also that the manuscript will not be submitted elsewhere while under consideration for publication in this journal.

Language. Articles in MEMOCS are usually in English, but articles written in other languages are welcome.

Required items. A brief abstract of about 150 words or less must be included. It should be selfcontained and not make any reference to the bibliography. If the article is not in English, two versions of the abstract must be included, one in the language of the article and one in English. Also required are keywords and a Mathematics Subject Classification or a Physics and Astronomy Classification Scheme code for the article, and, for each author, postal address, affiliation (if appropriate), and email address if available. A home-page URL is optional.

Format. Authors are encouraged to use $\mathrm{IAT}_{\mathrm{E} X}$ and the standard amsart class, but submissions in other varieties of $\mathrm{T}_{\mathrm{E}} \mathrm{X}$, and exceptionally in other formats, are acceptable. Initial uploads should normally be in PDF format; after the refereeing process we will ask you to submit all source material.

References. Bibliographical references should be complete, including article titles and page ranges. All references in the bibliography should be cited in the text. The use of $\mathrm{BIBT}_{\mathrm{E}} \mathrm{X}$ is preferred but not required. Tags will be converted to the house format, however, for submission you may use the format of your choice. Links will be provided to all literature with known web locations and authors are encouraged to provide their own links in addition to those supplied in the editorial process.

Figures. Figures must be of publication quality. After acceptance, you will need to submit the original source files in vector graphics format for all diagrams in your manuscript: vector EPS or vector PDF files are the most useful.

Most drawing and graphing packages - Mathematica, Adobe Illustrator, Corel Draw, MATLAB, etc. - allow the user to save files in one of these formats. Make sure that what you are saving is vector graphics and not a bitmap. If you need help, please write to graphics@msp.org with as many details as you can about how your graphics were generated.

Bundle your figure files into a single archive (using zip, tar, rar or other format of your choice) and upload on the link you been provided at acceptance time. Each figure should be captioned and numbered so that it can float. Small figures occupying no more than three lines of vertical space can be kept in the text ("the curve looks like this:"). It is acceptable to submit a manuscript with all figures at the end, if their placement is specified in the text by means of comments such as "Place Figure 1 here". The same considerations apply to tables.

White Space. Forced line breaks or page breaks should not be inserted in the document. There is no point in your trying to optimize line and page breaks in the original manuscript. The manuscript will be reformatted to use the journal's preferred fonts and layout.

Proofs. Page proofs will be made available to authors (or to the designated corresponding author) at a Web site in PDF format. Failure to acknowledge the receipt of proofs or to return corrections within the requested deadline may cause publication to be postponed. 
Mathematics and Mechanics of Complex Systems vol. 5 no. 2

On the well-posedness of the Green-Lindsay model

Gia Avalishvili, Mariam Avalishvili and Wolfgang $\mathrm{H}$. Müller

Linear pantographic sheets: Asymptotic micro-macro models identification

Claude Boutin, Francesco dell'Isola, Ivan Giorgio and Luca Placidi

Explicit formulas for relaxed disarrangement densities arising from structured deformations

Ana Cristina Barroso, José Matias, Marco Morandotti and David R. Owen

The general form of the relaxation of a purely interfacial energy for structured deformations

Miroslav Šilhavý

MEMOCS is a journal of the International Research Center for the Mathematics and Mechanics of Complex Systems at the Università dell' Aquila, Italy.

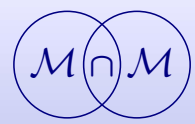

